Spectral Control of Diode Lasers Using External Waveguide Circuits

R.M. Oldenbeuving 


\section{Spectral Control of Diode Lasers \\ Using External Cavity Waveguide Circuits}

door

Ruud Michiel Oldenbeuving 


\section{Samenstelling van de promotiecommissie:}

\section{Voorzitter \& secretaris:}

Prof. dr. G. van der Steenhoven University of Twente, The Netherlands

\section{Promotor:}

Prof. dr. K.-J. Boller

University of Twente, The Netherlands

\section{Assistent-promotor:}

Dr. ir. H. L. Offerhaus

University of Twente, The Netherlands

\section{Leden:}

Prof. dr. A. P. Mosk

University of Twente, The Netherlands

Prof. dr. J. L. Herek

University of Twente, The Netherlands

Prof. dr. F. Bijkerk

University of Twente, The Netherlands

Prof. dr. C. Fallnich

Westfälische Wilhelms-Universität Münster, Germany

Prof. dr. U. Morgner

Leibniz Universität Hannover, Germany

Cover: False-color Scanning Electron Microscope (SEM) picture of the electrical wires and heaters on the optical waveguide chip, which is used as external cavity for diode laser control (see Chapter 2). Because the SEM is zoomed-out very far, electron beam distortion is caused by the SEM's focusing magnet. This produces the fish-eye effect in the picture.

Copyright (c) 2013 R.M. Oldenbeuving

ISBN: 978-90-365-3483-3

DOI: $10.3990 / 1.9789036534833$

Printed by Gildeprint Drukkerijen, Enschede, The Netherlands 


\section{Spectral Control of Diode Lasers Using External Waveguide Circuits}

\section{ProEFsCHRIFT}

ter verkrijging van

de graad van doctor aan de Universiteit Twente, op gezag van de rector magnificus, prof. dr. H. Brinksma,

volgens besluit van het College voor Promoties, in het openbaar te verdedigen op vrijdag 1 februari 2013 om 16.45 uur

door

\section{Ruud Michiel Oldenbeuving}

geboren op 10 oktober 1981

te Coevorden 
Dit proefschrift is goedgekeurd door:

De promotor: $\quad$ Prof. dr. K.-J. Boller

De assistent-promotor: Dr. ir. H. L. Offerhaus

The research presented in this thesis was carried out at the Laser Physics and Nonlinear Optics group, Department of Science and Technology, MESA+ Institute for Nanotechnology, University of Twente, P.O. Box 217, 7500 AE Enschede, The Netherlands. This research is supported by the Dutch Technology Foundation STW, which is part of the Netherlands Organization for Scientific Research (NWO) and partly funded by the Ministry of Economic Affairs (project number 10442). 
To my loving parents. 



\section{Summary}

In this Thesis we investigate spectral control of diode lasers using external waveguide circuits. The purpose of this work is to investigate such external control for providing a new class of diode lasers with technologically interesting properties, such as a narrow spectral bandwidth and spectrally tunable output in a hybrid integrated format. These lasers are of interest in a variety of scientific and industrial applications. To give a state-of-the-art example, we consider optical beam forming networks, that can be used in phased antenna arrays for satellite communications. These type of networks require spectral tuning and a narrow laser bandwidth to increase the spatial resolution of the antenna's signal [1-3]. In such microwave photonics applications, the option for an integration of entire arrays of lasers and waveguide circuits is required, as well as the option of multiple injection locking for a control of the optical phase of the output.

Another example is the simultaneous spectral and phase control of entire arrays of diode lasers. The superimposed output may offer the generation of trains of ultrashort pulses, which is equivalent with the generation of a phase locked comb of equidistant frequencies. Such sources can, for example, be of interest for high-speed optical data storage.

In the first chapter, we discuss the theoretical design considerations and spectral properties for a waveguide based external cavity semiconductor laser (WECSL). The essential requirements to realize single-frequency operation with a WECSL are analyzed. We investigated the required properties for the two main components that comprise the WECSL, which are the semiconductor gain chip and the frequency selective waveguide circuit that provides the external feedback. The specific implementation of a highly frequency selective feedback via two micro ring resonators (MRRs), based on available low loss $\mathrm{Si}_{3} \mathrm{~N}_{4} / \mathrm{SiO}_{2}$ waveguide technology, is described in more detail. The values for the design parameters in this implementation indicate that it is possible to obtain single-frequency oscillation across the entire telecommunication C-band (1530-1565 nm). Furthermore, we expect a narrow optical bandwidth from the WECSL, well below $1 \mathrm{MHz}$. More specifically, for the envisioned parameters of our implementation, we estimate the expected fundamental lower limit of the laser bandwidth (Schawlow-Townes limit) to lie in the order of tens of $\mathrm{kHz}$. We discuss from the scaling of this bandwidth with the waveguide circuit feedback parameters that the bandwidth limit may be significantly lowered increasing 
the finesse. If, by improvements in the design, the amount of losses in the external cavity can be lowered, the laser bandwidth could be decreased to a lower limit of about $10 \mathrm{~Hz}$.

To investigate if WECSLs can be applied in the mentioned example of optical beam forming networks, we consider injection locking which should provide extended spectral control, ultimately, of multiple WECSLs. This technique enables to externally control the optical phase of a laser oscillator by injecting light from another laser of much lower power. We expect the injection locking range of our WECSLs to be in the order of several hundreds of $\mathrm{MHz}$.

In the second chapter we present the experimental characterization of a WECSL. It consists of an anti-reflection coated diode laser as the gain element and a micro ring resonator (MRR) waveguide circuit which, due to its function as a spectrally selective feedback element, we call an MRR mirror. The MRR mirror comprises two slightly different micro ring resonators in series with radii $R_{1}=50 \mu \mathrm{m}$ and $R_{2}=55 \mu \mathrm{m}$, which yields a free spectral range (FSR) of $\Delta \lambda_{F S R, t o t}=42.3 \mathrm{~nm}$, a low reflection bandwidth of $0.2 \mathrm{~nm}$ and a sufficiently low reflectivity at undesired wavelengths.

Measurements showed that the WECSL is oscillating at a single frequency with a high SMSR of $50 \mathrm{~dB}$, and is widely tunable, over its full free spectral range of $42.3 \mathrm{~nm}$ via thermal tuning of the ring resonators. Switching between two arbitrary wavelengths takes only about $200 \mu \mathrm{s}$. The WECSL offers a small spectral bandwidth of $25 \mathrm{kHz}$. This measurement is in reasonable agreement with the currently available MRR mirror and coupling to the gain element. The agreement indicates that, via technical improvements, such as higher optical coupling between diode laser and waveguide, it should be possible to reduce the experimental bandwidth of the laser by several orders of magnitude.

To investigate if WECSLs can be applied in the mentioned example of optical beam forming networks, it is imperative to understand how the WECSL's spectral properties can be described for externally applied optical control via injection locking. The injection locking experiments of two individual WECSLs shows that the Q-factor of the WECSL was determined to be $7.7 \cdot 10^{4}$. This corresponds to a Schawlow-Townes limit for a Fabry Perot laser of $114 \mathrm{kHz}$, which is comparable to the earlier measured $25 \mathrm{kHz}$. This shows that spectral properties of a WECSL, which is optically controlled via injection locking, are well described with existing theories for Fabry Perot lasers.

In the third chapter we present and theoretically investigate an innovative laser mode locking scheme which is based on laser oscillation of an entire comb of frequencies, each of them being amplified in a separate gain medium. In mode locking of such a separate gain (SEGA) laser, the individual oscillators are mutually phase-locked by nonlinear feedback from a common semiconductor saturable absorber mirror (SESAM). As a result, ultra-short pulses are generated. This scheme offers a new route to mode locked lasers with high average output power, repetition rates that can be scaled into the $\mathrm{THz}$ range, and a bandwidth and pulse duration that can be dynamically controlled. We 
investigate the feasibility of SEGA mode locking using a numerical model. The calculations focus on finding a laser system and a set of physical properties of SESAMs, with which it might be possible to demonstrate SEGA mode locking for the first time. Within the range of required physical properties we identify a state-of-the-art SESAM that might be suitable for further experimental investigation.

In the fourth chapter, we present a set of preliminary experiments with the attempt to demonstrate SEGA mode locking. Two different setups are described, which are based on a diode laser bar with feedback from a SESAM via an external grating.

Measurements on the first setup showed a single occasion where an equidistant frequency comb with a frequency spacing of $66.8 \mathrm{GHz}$ was observed. This occasion also showed peaks in the intensity autocorrelation trace for the first time. However, the results suggested that the remaining nonlinearity in the dispersion of the feedback grating formed a problem to obtain a sufficiently precise equidistance of the generated frequency comb. Additionally, results could not be improved or reproduced, due to thermal damage of the used SESAM through operation with a reduced number of diode elements at an increased power.

To solve these difficulties we improved our setup by including an array of non-equidistant waveguides (pitch converter) in front of the diode bar. The purpose of such a converter is to effectively modify the pitch of the diode lasers in the diode bar and also offer, via tapered output ends of the waveguides in the pitch converter, a better focusability for operation at low average power. Equidistant frequency combs were achieved with two different pitch converters. With the first one, a $69.6 \mathrm{GHz}$ frequency spacing was achieved, equidistant for $60 \%$ of the spectrum within $5 \mathrm{GHz}$ precision, which is close to the experimental resolution limit. With the second pitch converter a frequency spacing of $20.8 \mathrm{GHz}$ was achieved, equidistant for $67 \%$ of the spectrum within the experimental resolution. The spectral measurements generally show frequency combs in which the degree of equidistance increases when a SESAM is used in the cavity rather than a plain mirror.

To inspect the temporal properties of the output with ultrafast time resolution, we measured the intensity autocorrelation (IA) with a setup that is based on collinearly phase matched second harmonic generation. The IA traces (second harmonic power vs. delay) show distinct peaks at delay times matching the average spectral spacing of the frequencies. However in all cases the peak to background ratio at zero delay time was much smaller than expected for a Fourier limited pulse train and, also, for a random superposition of the modes oscillating in the frequency comb. Calculations show that the IA measurements can be explained by an almost equidistant frequency comb with random phases, taking into account the measured power imbalance in the two arms of the autocorrelator and a somewhat incomplete beam overlap. When a mirror rather than a SESAM is used, similar IA traces are obtained with similar peak to background ratios at zero delay, which would indicate that operation with 
a SESAM does not accomplish any change in the relative phasing of the oscillating modes. However, when a mirror is installed in the laser, the peaks at non-zero delay decrease more rapidly than with a SESAM. The corresponding improvement of equidistance in the frequency combs can also be seen directly in the spectral measurements. The slight improvement in the degree of spectral equidistance in the frequency comb induced by the SESAM implies that the SESAM exerts some of the intended control, which is ultimately a fully equidistant spectrum through mode locking, to generate a pulsed output.

Further evidence that feedback from the SESAM contributes to nonlinear effects in the dynamics of the laser can be derived from measurements of the average output power vs. pump power. The output power is found to increase nonlinearly beyond a certain pump current, when the SESAM is present in the cavity. This is in contrast to what is observed when a plain mirror is used for feedback, where the output power increased linearly.

Although the currently available results are not conclusive, they also offer much insight into and bear promise for a demonstration of mode locking in subsequent experiments. Our measurements and calculations show that we are on the verge of an experimental demonstration of SEGA mode locking. By implementing improvements as suggested in Chapter 5, we hope to make an important step towards stable mode locking of separate gain lasers. This would be advantageous for possible applications of mode locked lasers where extremely high repetition rates are required in combination with a high average output power. 


\section{Samenvatting}

In dit proefschrift onderzoeken we de spectrale controle van diode lasers, die gebruik maken van externe golfgeleider circuits. De bedoeling van dit werk is, door deze externe vorm van controle te onderzoeken, een nieuwe klasse van hybride, geïntegreerde, diode lasers te maken. Deze lasers hebben namelijk technologisch interessante toepassingen, zoals spectrale smalbandigheid en afstembaarheid. Zulke lasers zijn interessant voor vele applicaties in zowel wetenschappelijke als industriële toepassingen. Om een high-tech voorbeeld te noemen, bekijken we een optisch bundelvormer netwerk, die gebruikt kan worden in een gefaseerde rij antenne voor satellietcommunicatie. Dit type netwerken hebben een laser nodig die spectraal smalbandig is en tegelijkertijd ook in golflengte afstembaar. Met die eigenschappen kan de spatiële resolutie van het signaal van de antenne verhoogd worden [1-3]. In zulke microgolf fotonische toepassingen, is de optie voor integratie van hele rijen van lasers en golfgeleider structuren nodig, alsmede de mogelijkheid om de fase van meerdere van deze lasers te controleren behulp van oscillatie synchronisatie via injectie van licht.

Een ander voorbeeld is het gelijktijdig controleren van het spectrum en de fase van volledige rijen van diode lasers. De gecombineerde lichtbundels kunnen de een trein van extreem korte pulsen opwekken, vergelijkbaar met een fase gesynchroniseerde frequentiekam met een gelijke afstand tussen de frequenties. Zulke lichtbronnen kunnen bijvoorbeeld interessant zijn voor optische dataopslag met hoge snelheden.

In het eerste hoofdstuk bespreken we de overwegingen voor het theoretische ontwerp en de spectrale eigenschappen van een golfgeleider-gebaseerd-externecaviteit diode laser (in het Engels afgekort tot WECSL). De essentiële eisen voor het realiseren van een WECSL die licht uitzendt met een enkele frequentie worden geanalyseerd. We hebben de vereiste eigenschappen onderzocht voor de twee belangrijkste componenten die de WECSL omvatten, namelijk de halfgeleider versterkings-chip en het frequentieselectieve golfgeleidercircuit dat voor de externe feedback zorgt. De specifieke implementatie van een uiterst frequentieselectieve feedback via twee microringresonatoren (MRR's), op basis van de beschikbare golfgeleider technologie $\mathrm{Si}_{3} \mathrm{~N}_{4} / \mathrm{SiO}_{2}\left(\right.$ TriPleX ${ }^{T M}$ ), wordt in meer detail beschreven. Uit de waarden voor de ontwerpparameters in deze uitvoering blijkt dat het mogelijk is om lichtoscillatie op een enkele frequentie te verkrijgen, verstembaar over de gehele telecommunicatie C-band (1530$1565 \mathrm{~nm})$. Verder verwachten wij een smalle optische bandbreedte van de 
WECSL, ruim onder de $1 \mathrm{MHz}$. Preciezer gezegd, voor de beoogde parameters van de implementatie schatten we dat de verwachte fundamentele ondergrens van de laser bandbreedte (de Schawlow-Townes limiet) in de orde van tientallen van $\mathrm{kHz}$ ligt. We bespreken aan de hand van het schalen van deze bandbreedte en het ontworpen golfgeleidercircuit hoe die de bandbreedte limiet aanzienlijk kan worden verlaagd door het verhogen van de finesse. Indien door verbeteringen in het ontwerp, het aantal verliezen in de externe caviteit kan worden verlaagd, zou de laser bandbreedte kunnen worden verminderd tot een ondergrens van ongeveer $10 \mathrm{~Hz}$.

Om te onderzoeken of WECSL's kunnen worden toegepast in het genoemde voorbeeld van optische bundelvormer netwerken, beschouwen we synchronisatie van licht via injectie die uiteindelijk de uitgebreide spectrale controle van meerdere WECSL's mogelijk moet maken. Deze techniek controleert de optische fase van een laser oscillator door injectie licht van een andere laser met veel lager vermogen. We verwachten dat het bereik van de synchronisatie van licht via injectie voor onze WECSL's in de orde van enkele honderden $\mathrm{MHz}$ ligt.

In het tweede hoofdstuk presenteren we de experimentele karakterisatie van een WECSL. De WECSL bestaat uit een antireflectie gecoate diodelaser als lichtversterkingselement en een microringresonator (MRR) golfgeleidercircuit, dat door zijn functie als een spectraal selectief feedback element door ons een MRR spiegel genoemd wordt. De MRR spiegel bestaat uit twee enigszins verschillende microringresonatoren in serie geschakeld en met een straal van $R_{1}=50 \mu \mathrm{m}$ en $R_{2}=55 \mu \mathrm{m}$, die een vrij spectraal bereik (FSR) levert van $\Delta \lambda_{F S R, t o t}=42.3 \mathrm{~nm}$, een lage reflectie bandbreedte van $0.2 \mathrm{~nm}$ en een voldoende lage reflectie voor ongewenste golflengten.

Metingen toonden aan dat de WECSL oscilleert op een enkele frequentie met een hoge SMSR van $50 \mathrm{~dB}$. Verder is hij breed afstembaar over het volledige vrij spectraal bereik van $42.3 \mathrm{~nm}$ via thermische verstemming van de ringresonatoren. Schakelen tussen twee willekeurige golflengten duurt slechts ongeveer $200 \mu$ s. De WECSL biedt een kleine spectrale bandbreedte van $25 \mathrm{kHz}$. Deze meting is redelijk in overeenstemming met de verwachtingen voor de gebruikte MRR spiegel en zijn koppeling met het versterkingselement. Dit geeft aan dat, via technische verbeteringen, zoals een betere optische koppeling tussen diodelaser en golfgeleider, het mogelijk moet zijn om de experimentele bandbreedte van de laser te verlagen met enkele ordes van grootte.

Om te onderzoeken of WECSL's kunnen worden toegepast in het genoemde voorbeeld van optische bundelvormer netwerken, is het noodzakelijk om te begrijpen hoe de spectrale eigenschappen van WECSL's kunnen worden beschreven voor externe optische controle door synchronisatie via injectie van licht. Uit de experimenten waarbij twee afzonderlijke WECSL's gesynchroniseerd werden, bleek de Q-factor van de WECSL $7.7 \cdot 10^{4}$ te zijn. Dit komt overeen met een Schawlow-Townes limiet voor een Fabry Perot laser van $114 \mathrm{kHz}$, wat vergelijkbaar is met de eerder gemeten $25 \mathrm{kHz}$. Dit toont aan 
dat spectrale eigenschappen van een WECSL, die optisch wordt gecontroleerd door synchronisatie via injectie van licht, goed beschreven kan worden met bestaande theorien voor Fabry Perot lasers.

In het derde hoofdstuk presenteren en onderzoeken we een innovatieve laser modus synchronisatie die is gebaseerd op laser oscillatie van de gehele kam van frequenties, welke allen worden versterkt in afzonderlijke versterkingselementen. Tijdens de synchronisatie van zo'n laser met ruimtelijk afzonderlijke versterking (in het Engels afgekort tot SEGA), worden de verschillende oscillatoren fase gesynchroniseerd door nietlineaire feedback van een gemeenschappelijke halfgeleider verzadigbare absorptie spiegel (SESAM). Daardoor worden extreem korte pulsen licht gegenereerd. Deze opzet biedt een nieuwe route naar de modus gesynchroniseerde lasers met hoge gemiddelde uitgangsvermogens, herhalingsfrequenties in het $\mathrm{THz}$ bereik en een bandbreedte en pulsduur die dynamisch kan worden aangestuurd. We onderzoeken de haalbaarheid van SEGA synchronisatie met behulp van een numeriek model. De berekeningen zijn gericht op het vinden van een laser systeem en een aantal fysische eigenschappen van SESAM's, waarmee voor het eerst SEGA synchronisatie experimenteel kan worden aangetoond. Binnen het bereik van de vereiste fysische eigenschappen identificeren we een state-of-the-art SESAM die mogelijk geschikt is voor verder experimenteel onderzoek.

In het vierde hoofdstuk presenteren we een set van inleidende experimenten met de bedoeling om SEGA synchronisatie aan te tonen. Twee verschillende opstellingen zijn beschreven, gebaseerd op een diode laser bar met feedback van een SESAM via een externe tralie.

Metingen op de eerste opstelling vertoonde één enkele gelegenheid waar een equidistante frequentie kam met een frequentie afstand van $66.8 \mathrm{GHz}$ werd waargenomen. Deze gelegenheid toonde pieken in de intensiteitsautocorrelatiegrafiek voor de eerste keer. Echter, de resultaten suggereerden dat de resterende niet-lineariteit in de dispersie van de tralie een probleem vormde, om een voldoende nauwkeurig equidistante frequentiekam te verkrijgen. Bovendien konden de resultaten niet worden verbeterd of gereproduceerd door thermische beschadiging van de gebruikte SESAM.

Om deze problemen op te lossen hebben wij onze opstelling verbeterd door een rij niet-equidistante golfgeleiders (pitch verdeler) voor de diode bar te plaatsen. Het doel van een dergelijke verdeler wijzigt in essentie de afstand tussen de diode lasers in de diode bar. Tegelijkertijd biedt de pitch verdeler via tapse uitgangseinden van de golfgeleiders een betere focusseerbaarheid, wat voordelig is bij lage gemiddelde vermogens. Equidistante frequentie kammen werden bereikt met twee verschillende pitch verdelers. Met de eerste is een $69.6 \mathrm{GHz}$ afstand is bereikt, equidistant over $60 \%$ van de spectra $5 \mathrm{GHz}$ precisie, wat dichtbij de experimentele resolutiegrens is. Met de tweede pitch verdeler is een frequentie afstand van $20.8 \mathrm{GHz}$ werd bereikt, equidistant over $67 \%$ van het spectrum binnen de experimentele resolutie. De spectrale metingen tonen in het algemeen frequentie kammen waarin de mate van equidistantie toeneemt 
wanneer een SESAM wordt gebruikt in vergelijk met een gewone spiegel.

Om de tijdsafhankelijke eigenschappen van het licht met ultrasnelle tijdsresolutie te inspecteren, meten we de intensiteitsautocorrelatie (IA) met een opstelling die gebaseerd is op collineaire fase afgestemde tweede harmonische generatie. De IA grafieken (tweede harmonische vermogen vs. vertraging) geven duidelijke pieken weer op vertragingstijden die overeenkomen met de gemiddelde spectrale afstand tussen de frequenties. Echter in alle gevallen was de piek tot achtergrond verhouding bij nul vertraging was veel kleiner dan verwacht voor een Fourier gelimiteerde pulstrein, alsmede voor een willekeurige superpositie van de oscillerende modi in de frequentiekam. Uit berekeningen blijkt dat de IA metingen kunnen worden verklaard door een bijna equidistante frequentiekam met willekeurige fases, waarbij rekening wordt gehouden met de gemeten vermogensongelijkheid in de twee armen van de autocorrelator en een enigszins onvolledige bundeloverlap. Indien een spiegel in plaats van een SESAM wordt gebruikt, worden soortgelijke IA grafieken verkregen met vergelijkbare piek tot achtergrond verhoudingen op nul vertraging. Dit doet vermoeden dat het gebruik van een SESAM geen verandering in de relatieve spreiding van de oscillerende modi bewerkstelligt. Wanneer echter een spiegel is geïnstalleerd in de laser, neemt de hoogte van de pieken bij niet-nul vertraging sneller af dan met een SESAM. De overeenkomstige verbetering van gelijke afstand in de frequentie kammen is ook direct zichtbaar in de spectrale metingen. De lichte verbetering in de mate van spectrale gelijke afstand in de frequentiekam geïnduceerd door de SESAM impliceert dat de SESAM een deel van de beoogde controle uitoefent, wat een uiteindelijk tot volledig equidistant spectrum via modus synchronisatie moet leiden.

Verder bewijs dat de feedback van de SESAM bijdraagt aan niet-lineaire effecten in de dynamiek van de laser kan worden afgeleid uit metingen van het gemiddelde uitgangsvermogen vs. pompvermogen. Het uitgangsvermogen blijkt lineair toe te nemen boven een bepaalde pomp stroom, wanneer de SESAM aanwezig is in de caviteit. Dit in tegenstelling tot wat wordt waargenomen wanneer een gewone spiegel voor feedback wordt gebruikt, waarbij het uitgangsvermogen lineair toeneemt.

Hoewel de momenteel beschikbare resultaten niet overtuigend zijn, bieden ze toch veel inzicht en bieden ze goede vooruitzichten voor een demonstratie van de modus synchronisatie in vervolgexperimenten. Onze metingen en berekeningen laten zien dat we op de drempel staan van een experimentele demonstratie van SEGA synchronisatie. Door de implementatie van verbeteringen, zoals voorgesteld in hoofdstuk 5 , hopen we een belangrijke stap te maken in de richting van een stabiele modus synchronisatie van afzonderlijke. Dit zou voordelig zijn voor mogelijke toepassingen van modus gesynchroniseerde lasers waar extreem hoge herhalingsfrequenties zijn vereist in combinatie met een hoog gemiddeld uitgangsvermogen. 


\section{Contents}

Summary vii

Samenvatting $\quad x i$

1 Introduction $\quad 1$

2 WECSL theoretical aspects $\mathbf{5}$

2.1 Introduction . . . . . . . . . . . . . . . 5

2.2 External cavity semiconductor lasers . . . . . . . . . . . . 7

2.2 .1 Gain section . . . . . . . . . . . . . 7

2.2 .2 MRR mirror design . . . . . . . . . . . . . . . . . . . . . . . . . . .

2.2 .3 Laser bandwidth . . . . . . . . . . . . . . . 13

2.3 WECSL design . . . . . . . . . . . . . . . . . 16

2.4 Injection locking . . . . . . . . . . . . . . . . . . . 21

2.4.1 Injection locking principle . . . . . . . . . . 22

2.5 Conclusions . . . . . . . . . . . . . . . . . . . . . 24

$\begin{array}{llr}3 & \text { WECSL experiments } & \mathbf{2 7}\end{array}$

3.1 Introduction . . . . . . . . . . . . . . . . . 27

3.2 Characterization of waveguide circuits . . . . . . . . . . 29

3.3 Characterization of the free-running diode laser . . . . . . . . 31

3.4 WECSL characterization . . . . . . . . . . . . . . 32

3.4 .1 Optical characterization . . . . . . . . . . 32

3.4 .2 Injection locking . . . . . . . . . . . . . . . 38

3.5 Conclusions . . . . . . . . . . . . . . . . . . . . . . 42

4 SEGA mode locking theory 43

4.1 Introduction . . . . . . . . . . . . . . . . . . 43

4.2 Standard mode locking . . . . . . . . . . . . . . . . . . . . . . 45

4.3 Concept . . . . . . . . . . . . . . . . . . . . 47

4.4 Model ......................... 49 
4.4 .1 Frequency spacing ... . . . . . . . . . . . . 50

4.4 .2 Gain model . . . . . . . . . . . . . . . . 53

4.4 .3 Temporal dynamics ................. 56

4.4.4 Initial conditions and evaluation ......... 58

4.5 Results . . . . . . . . . . . . . . . . . . . . . 58

4.6 Conclusions . . . . . . . . . . . . . . . . 65

$\begin{array}{lll}5 & \text { SEGA mode locking experiments } & 67\end{array}$

5.1 Introduction . . . . . . . . . . . . . . . 67

5.2 Initial setup . . . . . . . . . . . . . . . . . 68

5.3 Measurement methods . . . . . . . . . . . . . . . . 70

5.4 Initial measurements . . . . . . . . . . . . . . . . . . 75

5.5 Improved setup . . . . . . . . . . . . . . . . . . 80

5.6 Improved setup measurements and results . . . . . . . . . . . . 83

5.7 Conclusions . . . . . . . . . . . . . . . . . . . 88

$\begin{array}{llr}6 & \text { Conclusions } & 91\end{array}$

$\begin{array}{ll}\text { A Appendix A } & 95\end{array}$

$\begin{array}{lr}\text { Bibliography } & 99\end{array}$

$\begin{array}{lr}\text { Publications } & 105\end{array}$

$\begin{array}{lr}\text { Dankwoord } & 109\end{array}$ 


\section{1 \\ Introduction}

Ever since humans roamed the earth, they have been fascinated by the color of light. Initially, all efforts to control the color of light were based on optical filtering, such as simple reflection off paint, passing light through colored glass or diffraction at fine periodic structures. Optical filtering has the advantage of improving the spectral purity however it has the disadvantage that highest purity is only achieved with severe decrease of the overall optical power.

A major breakthrough removing this fundamental limitation was the invention of the laser [4]. Based on stimulated emission of light, in combination with optical filtering, one could assert control on any or multiple of the properties of light, such as amplitude, phase, frequency, polarization and spatial distribution at, basically, all desired power levels. As example, with a suitable combination of amplitude, polarization, phase and frequency of light, also the temporal structure of light can be controlled, e.g., to generate trains of ultrashort pulses.

Fascinatingly, and unknown to most people, current popular demands for increasing the speed of computers and internet connections have much to do with controlling the spectral and temporal properties of light. The reason is that the capabilities of current electronics are approaching their ultimate limits when attempting to increase the speed of data transfer in cable and freespace (e.g., microwave) internet connections, or when attempting to speed-up the rates of computing and data storage. As examples of current electronics, typical fastest consumer solid-state hard drives operate with a maximum speed of about 4 to $6 \mathrm{Gbit} / \mathrm{s}$ [5], while internet connections are already addressed in the IEEE standards with up to $100 \mathrm{Gbit} / \mathrm{s}$ [6], although these speeds are not yet commercially available. In fact, the fastest available internet connections currently commercially available are so-called fiber-to-the-home connections, which are already based on optical fiber communications, and provide data rates of up to $500 \mathrm{Mbit} / \mathrm{s}$ [7]. In general it can be observed that, mostly where 
electronic components do not meet the high requirements for future speed or density of data, advanced light sources are expected to play a dominant role by providing output with high spectral purity, wide wavelength tuning, phase control or temporal control. As examples of optics lifting the restraints of current electronics, we name three envisioned routes in applications. First, it appears possible to dramatically increase the writing speed in magnetic storage devices by using ultrashort light pulses in stead of magnetic fields. Specifically, it has been shown with single, intense optical pulses that a magnetic bit can be switched in a time shorter than $100 \mathrm{fs}$ [8]. When being able to repeat such switching with a sufficiently high optical pulse rate, this would correspond to a maximum read/write operation speed of over $1 \mathrm{Tbit} / \mathrm{s}$. The required optical pulse train does not exist yet, due to its rather unusual combination of properties which is a huge repetition rate of $1 \mathrm{THz}$ and an appreciable average power of $10 \mathrm{~W}$ focused down to a diffraction limited spot [8].

While there is no fundamental problem in providing such properties, the envisioned applications impose strict technological requirements, especially the potential for miniaturization via integration, which has prevented such sources from realization so far. Having a laser that produces THz-levels of repetition rate also would allow for a fiber communication with such high speeds. However, electronic switching which is required to make a distinction between the zeros and ones in a stream of bits is not fast enough to accomplish these impressive speeds. Again, optics can lift the restraints of electronics, in this case by all-optical switching through use of the Kerr effect. In single switching events using laser pulses with relatively low $(\mathrm{kHz}$ to $\mathrm{MHz})$ repetition rates, ultra fast switching times in the femtosecond range have been demonstrated $[9,10]$. However, again, light sources that can offer such pulses with THz-level repetition rates, and bear the potential for integration on a chip, are not available. Also in situations where transmission in free space is required, such as currently done via microwaves, optical methods of data processing promise to be superior in performance. An example is advanced satellite communication using phased array antennas in which rapid sweeping of the direction of reception independent of the specific shape and orientation of the antenna can be achieved with optical delay lines. As with the previously described examples it will become necessary to realize large arrays of laser sources in a miniaturized format on a chip $[1,2]$. The spectral bandwidth of these lasers has to be extremely narrow, oscillating at the same frequency with a well-defined relative optical phase.

So far, we did not refer to any specific type of laser required for the named examples. However, there is only a single type of laser that seems to have the highest prospect, namely the diode laser. The reason can be found in the large degree of technological maturity of such lasers, which enables the possibility of combining a wide wavelength tunability, narrow optical bandwidth, with the possibility for mass production, low manufacturing costs and small size suitable for integration with waveguide circuits.

In this Thesis we have investigated two main diode laser configurations that use an external cavity and integrated optics. Because the intended applications 
of these two configurations have very different demands with regard to the spectral properties of the lasers, the used external cavities are quite different indeed. The first configuration may find applications mainly in data transfer, where we use the example of advanced satellite communication as possible utilization. To control the optical bandwidth and wavelength, we equip the diode laser with an external cavity that is formed by a frequency selective waveguide circuit integrated on a waveguide chip. The second configuration may find applications in the named high speed optical switching with highest repetition rates, and it may also form a key to extremely fast data writing and reading. We propose to use an array of diode lasers, where in the first step a free-space external cavity configuration is intended to control both the wavelength and phase of the individual diode lasers. Combining the output of all these lasers could theoretically lead to a train of ultra-short pulses with repetition rates in the range of hundreds of $\mathrm{GHz}$ or even $\mathrm{THz}$.

For the first type of application requiring narrow spectral bandwidth, currently a main approach is monolitically integrated semiconductor lasers, such as distributed Bragg reflector (DBR) lasers and distributed feedback (DFB) lasers. These lasers posses important technological advantages which are a small size, direct operation with electric pumping and the possibility of low cost fabrication in high numbers in a chip format. However, the bandwidth of $\mathrm{DBR}$ and DFB lasers is typically rather large, a few $\mathrm{MHz}$, and rapid wavelength tuning is possible only over the range of a few nanometers $[11,12]$. DBR and DFB lasers may be fabricated such that some of the optical properties can be improved, at the expense of others. As example, the optical bandwidth can be decreased, at the expense of tuning range and tuning speed. However, to date, a real commercially viable alternative providing ultra narrow bandwidth with high wavelength agility in a chip-sized format does not exist.

In this Thesis we investigate such an alternative, by coupling a diode laser to a frequency tunable integrated optics external cavity. We incorporate so-called micro-ring resonators (MRRs) as tunable spectral filters in an external cavity layout. In Chapter 2 of this thesis we describe in detail how to design such MRRs in integrated optic waveguides, in a layout which we call an MRR mirror. To form a laser, the MRR mirror is coupled to a semiconductor gain material. We call this hybrid approach a waveguide based external cavity semiconductor laser (WECSL).

The WECSL that we investigate here, is capable of achieving a bandwidth that is very narrow, currently around $25 \mathrm{kHz}$, as we show in Chapter 3. We also present measurements on a wide tuning range (full telecommunication Cband: $1530-1565 \mathrm{~nm})$ and fast switching between wavelengths $(200 \mu \mathrm{s})$. For the named application in advanced satellite communications using phased antenna arrays, we have investigated the injection locking [13] properties of our laser. Within a limited range, two separate WECSLs were injection locked and found to have an equal output frequency which is a signature for a fixed phase relation.

For the second type of applications, requiring ultrashort pulses with $\mathrm{THz}-$ 
level repetition rates and high average output power at the 10-Watt-level, one might consider standard passive mode locking techniques. However it turns out that such unusual combinations of repetition rates, output powers, and pulse durations are, to this date, difficult to achieve. Specifically, higher average powers generally require larger gain lengths.

To illustrate the resulting incompatibility, let us consider a very short cavity length of only a few hundred $\mu \mathrm{m}$, such as can be realized in semiconductor lasers. Indeed, very high repetition rates of hundreds of $\mathrm{GHz}$ are obtained with such lasers [14]. However, the short cavity length limits the roundtrip gain and thus also the average power, typically to the milliwatt range with such semiconductor lasers. Extending the cavity length allows for higher average output power, but also lowers the repetition rate.

In this Thesis, we describe a novel mode locking method that is capable of overcoming the named limitations by providing high repetition rates as well as high average output powers, by decoupling the repetition rate from the length of the cavity [15]. In this scheme, we consider a large set of single-frequency continuous-wave lasers that oscillate by amplification in spatially separated gain media. Mutual phase-locking, i.e., mode locking would then be achieved via nonlinear feedback such as from a common saturable absorber mirror. As a result, ultra-short pulses are generated. We call this approach separate gain (SEGA) mode locking. Compared to standard mode locking techniques, the new scheme offers three significant benefits. The light that is amplified in each separate gain element is spectrally narrowband and continuous wave, thereby avoiding issues related to group-velocity dispersion and nonlinear effects that can perturb the pulse shape. Such separately operated continuous-wave lasers also bear the potential to become integrated in a compact on-chip format, which is a clear advantage in the envisioned applications. The spectral separation of the set of frequencies on which the laser oscillates, and therefore the pulse repetition rate, is controlled by the dispersion of resonator-internal optical elements and the resonator geometry, not by the cavity length.

So far we have only theoretically investigated the feasibility of such a laser in Chapter 4. An according experimental setup is presented in Chapter 5 where we report on initial experimental investigations. 


\section{2 WECSL theoretical aspects}

In this chapter we present the design considerations and the expected spectral properties for a waveguide based external cavity semiconductor laser (WECSL). Applications and challenges in external cavity lasers are described in Section 2.1. The basics of waveguide based external cavity semiconductor lasers are addressed in Section 2.2. This section also provides an estimate for the minimum possible bandwidth of such lasers, with regard to the standard SchawlowTownes limit. Section 2.3 addresses a specific WECSL design with parameters based on a certain available gain material and waveguide technology. In Section 2.4 injection locking of WECSLs is briefly explained.

\subsection{Introduction}

Tunable diode lasers with narrow spectral bandwidths below $1 \mathrm{MHz}$ find applications in, e.g., coherent optical communications, where frequency tunability and narrow bandwidths can be used to increase data transfer density [16]. A potential application of high interest is found in broadband, phased array antenna systems, where the narrow laser bandwidth increases the spatial resolution of the antenna's signal and where laser wavelength tuning is necessary for phase retrieval $[1,2]$. In these applications, alternatively, a larger number of spectrally controlled diode lasers with narrow bandwidths in the $\mathrm{kHz}$-range, integrated in a mm-size format might be required [2, 3]. Free-space external cavity diode lasers are also capable of producing such narrow bandwidths, but their wavelength tuning is fairly slow (tens of milliseconds [17]), due to mechanical parts, such as moving gratings or feedback mirrors [18]. Also, their size is too large for integration.

The lower limit for the spectral bandwidth of free-running diode lasers 
without frequency selective feedback is in the order of GHz. Such values are found for the fundamental limit using the Schawlow-Townes theory for diode lasers [19], as will be discussed in more detail in Section 2.2. In order to reach bandwidths well below $1 \mathrm{MHz}$, spectral narrowing methods have to be applied. Such narrowing, in combination with spectral tuning, can be reached through tunable frequency selective optical feedback [19]. The two main approaches are monolithic semiconductor lasers, such as, on the one hand, distributed Bragg reflector (DBR) lasers or distributed feedback (DFB) lasers, and external cavity semiconductor lasers (ECSL) on the other hand. The bandwidth of DBR and DFB lasers is typically a few $\mathrm{MHz}$ and wavelength tuning (within microor nanoseconds) is possible over the range of a few nanometers $[11,12]$. DBR and DFB lasers are not suitable if a narrower bandwidth combined with wider tunability is required. ECSLs can reach bandwidths well below $1 \mathrm{MHz}$ and wide tunability $[11,12,18,19]$, if fluctuations in the optical cavity length are avoided, which is mechanically challenging. The Schawlow-Townes limit can only be reached by removing acoustic perturbations and by avoiding any minute errors in the positioning of the optical components inside the cavity [18]. Unfortunately, a mechanically stable cavity will result in lower tuning speed and tuning range.

In this chapter an innovative type of external cavity diode laser is presented, following the approach of Chu et al. [20, 21], where an optical gain chip is coupled to an external cavity that is integrated on a waveguide chip. We show that this approach, which we call a waveguide based external cavity semiconductor laser (WECSL), can provide highly frequency selective, and widely tunable feedback to the laser diode, to impose single frequency oscillation with narrow bandwidth, high wavelength coverage and high wavelength agility. To achieve such properties, we chose the waveguide chip to incorporate a double microring resonator (MRR) structure, as depicted in Fig. 2.1, which we refer to as an MRR mirror. The resonance frequencies of both MRRs can be tuned by heating, resulting in faster tunability $(200 \mu \mathrm{s})$ than possible for mechanically tuning a free-space external cavity. Since the external cavity is an integrated waveguide chip, and the diode laser is in firm mechanical contact with the waveguide (such as in hybrid integration), mechanical stability is significantly increased compared to free-space external cavities.

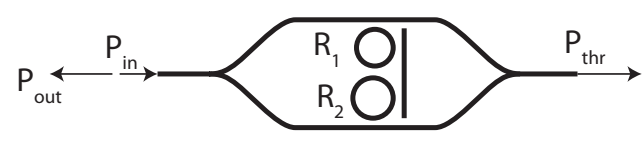

Figure 2.1: Schematic layout of an MRR mirror with two differently sized MRRs with radii $R_{1}$ and $R_{2}$. The black lines represent waveguides. $P_{\text {in }}$ indicates the power of the light sent into the input port of the MRR mirror, $P_{\text {out }}$ indicates the power reflected by the MRR mirror and $P_{\text {thr }}$ is the transmitted power. 
To provide an understanding of the basic design conditions for achieving proper spectral properties of a WECSL, we begin by presenting the relevant characteristics of the two main constraints in Section 2.2. The generation of spectrally selective feedback with integrated MRRs is presented with the purpose to employ this as an MRR mirror in the WECSL. The influence of various MRR design parameters on the spectral reflectivity are described, with the goal to achieve single-frequency operation. We show how these design parameters should yield a narrow spectral bandwidth of the laser output, in terms of the fundamental Schawlow-Townes limit. Following these general design considerations, in Section 2.3 we present the parameters that we have chosen for a specific realization of a WECSL. By combining a diode gain chip operating across the telecommunication C-band (1530-1565 nm) with a feedback chip based on $\mathrm{Si}_{3} \mathrm{~N}_{4} / \mathrm{SiO}_{2}$ waveguide technology (TriPleX ${ }^{T M}$ ) [22] with a boxshaped cross section, widely tunable, single frequency oscillation with tens of $\mathrm{kHz}$ spectral bandwidth is expected. Corresponding experimental results will be presented in Chapter 3.

An extended spectral control which comprises also the optical phase appears attractive for employing WECSLs in coherent communication techniques. We discuss in Section 2.4 a spectral and phase control of WECSLs via so-called injection locking. Also here we present corresponding experimental results in Chapter 3.

\subsection{External cavity semiconductor lasers}

In designing a frequency tunable, narrow bandwidth WECSL, some basic properties of the constituting components have to be taken into consideration. These will be discussed in the following subsections.

\subsubsection{Gain section}

For the proper operation of a WECSL, optical gain has to be provided from a semiconductor chip which is specially prepared for external optical feedback. In contrast, standard Fabry Perot diode laser chips are usually manufactured with a high-reflection coated back facet and, to form a monolithic resonator, the front facet is coated to achieve sufficiently high optical feedback. Due to the large gain per round trip that can be provided in a semiconductor chip (sometimes reaching factors in the order of $10^{6}$ ), diode lasers can easily each the oscillation threshold in spite of huge internal waveguide and re-absorption losses and with relatively low feedback from the facets. These huge gain factors make semiconductor gain chips particularly attractive for highly spectrally selective external feedback, even if the feedback seems comparatively weak, with values at the percent-level or below.

Due to the high value of the refractive index for many of the commonly used materials $\left(3<n_{d}<3.5\right)$, the Fresnel reflection from the output facet is about 
25\%-30\%. This reflection $R_{s}$ can be calculated via the Fresnel equations for reflection at an interface between two media (assuming normal incidence)

$$
R_{s}=\left|\frac{n_{d}-n_{a i r}}{n_{d}+n_{a i r}}\right|^{2},
$$

with $n_{d}$ the refractive index of the semiconductor material used, and $n_{\text {air }}$ the refractive index of air.

Without any further measures, due to the wide gain bandwidth (typically up to $10 \%$ of the absolute wavelength), Fabry-Perot diode lasers show oscillation simultaneously at multiple frequencies. The purpose of external frequency selective feedback, combined with a reduction of reflectivity at the diode's front facet, is to suppress such multi-mode oscillation and enable oscillation at other, selectable frequencies. For a success of this approach it is clear that the external feedback at a single selected frequency has to dominate a residual feedback from the front facet.

To let the laser output spectrum be dominated by the external cavity, rather than by feedback from the front facet, the standard approach is to have the front facet anti-reflection (AR) coated. Typical values for the residual reflection after $\mathrm{AR}$ coating are $10^{-2}-10^{-3}$. To reduce the reflectivity even further, the front facet can be angle cleaved, so that reflections have an angular mismatch with the diode laser's waveguide mode, thereby further decreasing undesired feedback. The residual reflection, expressed by a reflection factor, $R_{a}$, can be estimated using Eq.(2.2), which has been derived in the context of angular misalignment of Gaussian beams when coupling into a single-mode fiber [23]:

$$
R_{a}=e^{-\varsigma^{2}}
$$

where

$$
\varsigma=\frac{n_{d} \pi w_{0} \sin (2 \cdot \varphi)}{\lambda},
$$

with $w_{0}$ the beam radius at the facet (FWHM of the intensity) of the Gaussian beam, $\varphi$ the angle of the diode laser waveguide with the normal of the facet (clave angle) and $\lambda$ the wavelength of the laser in vacuum. Note that, in Eqs. $(2.2,2.3)$ the cleave angle is multiplied with a factor of two, to yield the total deflection angle. Due to the exponential in Eq.(2.2), even in the case of a small cleave angle, the amount of back reflection from the front facet into the diode laser waveguide quickly drops below the typical numbers of AR coating. To illustrate this with an example with typical values for the index $n_{d}=3.5$, and the beam radius in the facet plane $w_{d, x}=w_{d, y}=3 \mu \mathrm{m}$ the reflectivity $R_{a}$ drops below $10^{-3}$ at an angle of $\varphi=3.5^{\circ}$.

The maximum allowed residual reflectivity of the front facet of the diode laser, such that the diode laser's spectral properties are governed by the external feedback, depends on the gain provided by the diode laser and on the external 
feedback $R_{\text {ext }}$. Based on the complex field rate-equations, one can calculate the allowed maximum front facet reflection $R_{f}[24]$. As the approximate result, $R_{f}$ has to be smaller than $R_{\text {ext }}$, divided by the so-called linewidth-enhancement factor $\alpha$ [19], the latter being a semiconductor material property ${ }^{1}$,

$$
R_{f}<\frac{R_{e x t}}{\alpha^{2}}
$$

For diode lasers a typical number for the linewidth-enhancement factor is between 4 and $8[18,19]$. Therefore, one requires typically that the residual facet reflectivity is a factor of 15 to 50 lower than the external feedback.

\subsubsection{MRR mirror design}

The gain spectrum of a typical diode laser for telecommunications extends over several tens of nanometers. This may lead to undesired oscillation at multiple frequencies that belong to other longitudinal modes of the laser resonator. The ratio in power between the desired laser mode and these undesired modes is called the side-mode suppression ratio (SMSR). The side-mode suppression ratio is decreased when multiple modes are emitted by the laser. In the following, we show how to avoid these negative consequences via an appropriate design of the external cavity using a frequency selective feedback element, in our case micro ring resonators (MRRs). The spectral properties of the MRRs have to be carefully chosen such that feedback over most of the gain bandwidth is avoided, except for a selected, narrowband wavelength range of laser operation.

Consider an MRR mirror with a single MRR, as schematically depicted in Fig. 2.2a. The calculated reflectivity vs. input wavelength is illustrated in Fig. 2.2b for a typical set of fabrication parameters. Each peak in reflectivity represents a resonant frequency of the MRR. The spectral distance between adjacent peaks, called the free spectral range (FSR) is denoted as $\Delta \lambda_{F S R}$. The spectral bandwidth at full width half maximum (FWHM), which is approximately the same for all resonances shown, is denoted as $\Delta \lambda_{F W H M}$.

\footnotetext{
${ }^{1}$ The origin of the linewidth-enhancement factor $\alpha$ will be discussed in more detail in Section 2.2 .3
} 
a)

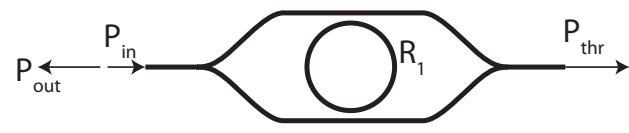

b)

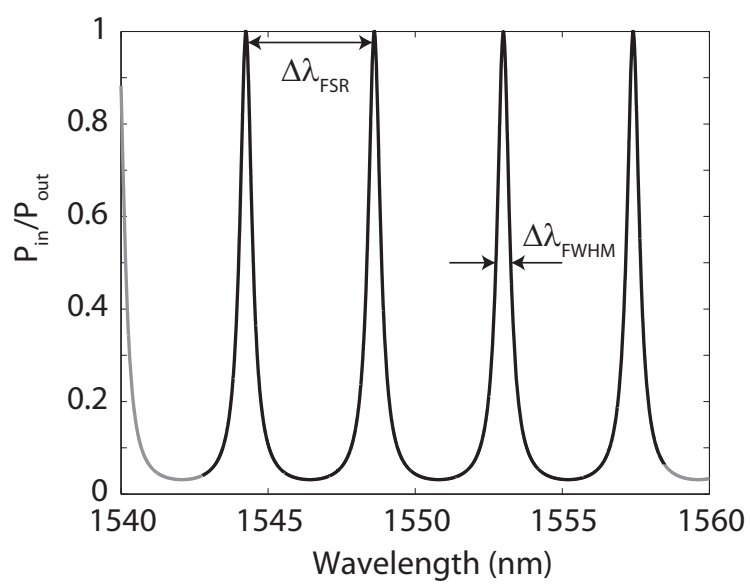

Figure 2.2: (a) schematic layout of an MRR mirror with a single $M R R$ with radius $R_{1}$. Single frequency light sent into the input waveguide (with power $P_{\text {in }}$ ) will be reflected if the wavelength is resonant with the MRR. (b) reflected power $\left(P_{\text {out }}\right)$ vs. input wavelength calculated for a typical $\mathrm{Si}_{3} \mathrm{~N}_{4} / \mathrm{SiO}_{2} \mathrm{MRR}$ with radius $\mathrm{R}_{1}=50 \mu \mathrm{m}$, neglecting for simplicity any losses. Each peak represents a resonant wavelength of the MRR. The spectral bandwidth (at FWHM) of a resonance is denoted as $\Delta \lambda_{F W H M}, \Delta \lambda_{F S R}$ denotes the free spectral range.

The FSR of a single MRR can be calculated by [25]

$$
\Delta \lambda_{F S R}=\frac{\lambda_{0}^{2}}{n_{g} L},
$$

where $\lambda_{0}$ is one of the resonant wavelengths (in vacuum), $n_{g}$ is the group index of the waveguide (containing form and material dispersion) and $L$ is the geometrical roundtrip length of the MRR. By changing the optical roundtrip length, $n_{g} \cdot L$, the resonant frequencies are shifted. In our case, the refractive index is varied by heating of the MRR via an electric heater, e.g., deposited as a thin-film wire, placed above the MRR. The MRRs used in this work (with $\mathrm{L}$ in the order of $350 \mu \mathrm{m}$ ) can be tuned over one full FSR via increasing the temperature by several hundreds of Kelvin. The $\mathrm{Si}_{3} \mathrm{~N}_{4} / \mathrm{SiO}_{2}$ waveguides used in this work, having a box-shaped cross section, possess a group index close to $n_{g}=1.73$, resulting in a typical tuning range of $\sim 4 \mathrm{~nm}$ around a wavelength of $1550 \mathrm{~nm}[22]$.

To extend the tuning range beyond one FSR, a second MRR with a slightly 
different radius (and thus different FSR) can be added in series (see Fig. 2.3a), to exploit the Vernier effect [26, 27]. Specifically, by choosing two FSRs, that differ by a small fraction, $\epsilon$, the total free spectral range becomes increased by a factor of $1 / \epsilon$. Fig. 2.3 shows, as an example, the calculated reflectivity spectrum for a double MRR mirror with a $10 \%$ difference in FSR. It can be seen that the wavelength spacing between the main transmission peaks, the free spectral range is increased by a factor of 10 , to about $40 \mathrm{~nm}$. This value is comparable to the width of the diode laser gain profile and appears suitable to restrict oscillation to a single, selected wavelength.

a)

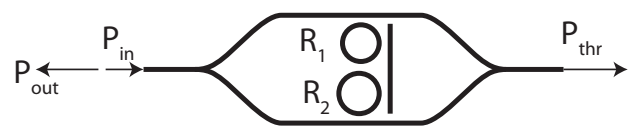

b)

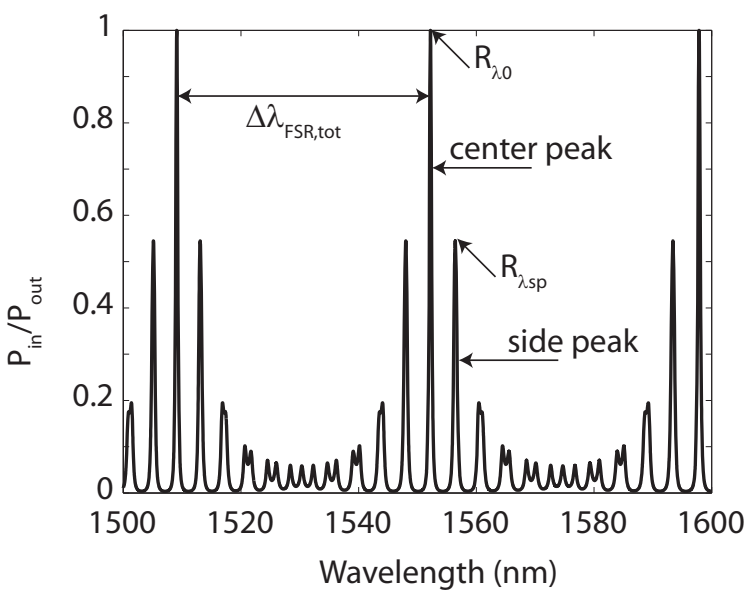

Figure 2.3: (a) schematic layout of an MRR mirror with two differently sized MRRs with radii $R_{1}$ and $R_{2}$. Single frequency light sent into the input waveguide (with power $P_{\text {in }}$ ) will be reflected if the wavelength is resonant with both MRRs. (b) reflected power $\left(P_{\text {out }}\right)$ vs. input wavelength calculated for a typical $\mathrm{Si}_{3} \mathrm{~N}_{4} / \mathrm{SiO}_{2}$ double $\mathrm{MRR}$ mirror with radii for this example $R_{1}=50 \mu \mathrm{m}$ and $R_{2}=55 \mu \mathrm{m}$, for a waveguide group index of $n_{g}=1.73$ and all $\kappa^{2}=0.4$, neglecting for simplicity any losses. $\Delta \lambda_{F S R \text {, tot }}$ denotes the total free spectral range. $R_{\lambda_{0}}$ denotes the reflectivity of the center peak and $R_{\lambda_{s p}}$ denotes the reflectivity of the side peak.

Of high importance for such selection is also the spectral bandwidth of the reflectivity peak. To determine this bandwidth with an analytical approximation, let us first consider again a single MRR. The spectral bandwidth (FWHM) of the reflection resonances of a single MRR, as given by Yariv [25], is described by 


$$
\Delta \lambda_{F W H M}=\frac{\lambda_{0}^{2}}{\pi L n_{g}} \cdot \frac{\kappa^{2}}{\sqrt{1-\kappa^{2}}} .
$$

In this expression, $\kappa$ is a coefficient which describes the coupling of the electric field amplitude from the input waveguide to the MRR and, respectively, from the MRR back into the output waveguide. Eq.(2.6) only incorporates the MRR's roundtrip losses via $\kappa$, while neglecting other losses (such as scattering, absorption and radiative bending losses). This is a valid approximation for the MRRs used in this research since, for the typical loss values discussed below, the coupling coefficient of the MRR accounts for over $99 \%$ of the roundtrip losses.

Now turning back to the double MRR, it can be seen that the reflectivity spectrum contains a number of side peaks with the highest side peaks directly adjacent to the main reflectivity peaks. When aiming at single frequency oscillation it has to be made sure in designing the double MRR feedback spectrum, that undesired oscillation at a side peak is suppressed. This can be achieved by ensuring that the roundtrip product of gain and feedback for the highest side peaks, $G_{\lambda_{s p}} \cdot R_{\lambda_{s p}}$, remains smaller than the corresponding product for the main peak, $G_{\lambda_{0}} \cdot R_{\lambda_{0}}$. Here $R_{\lambda_{0}}, R_{\lambda_{s p}}, G_{\lambda_{0}}$ and $G_{\lambda_{s p}}$ are the reflectivity and gain values at the wavelength that receives the highest feedback (called center peak) with that of the next peak in the reflectivity spectrum (called side peak), respectively (see also Fig. 2.3b). The ratio between the side peak and the center peak will be called peak-height ratio (PHR) and is given by $R_{\lambda_{s p}} / R_{\lambda_{0}}$. While the ratio of gain for the center wavelength to gain at its side peak is given by the curvature of the gain profile of the diode laser, the PHR is dependent on the bandwidth of the individual ring resonators (via $\kappa$ ) and their length deviation $(\epsilon)$.

For calculating the PHR the spectral shape of the resonances of the individual MRRs has to be taken into account. For simplicity, we have only considered the case for a single side peak. However, for completeness, it should be noted that - if the FWHM of the two MRRs are assumed equal - the first two side peaks occur at $\lambda_{s p}=\lambda_{0} \pm\left(\left|\Delta \lambda_{F S R, 1}-\Delta \lambda_{F S R, 2}\right| / 2\right)$.

The shape of the transmission resonances of a single MRR can be approximated by a Lorentzian shape $L(\lambda)$ (see, e.g., Little et al. [28])

$$
L(\lambda)=\left(\frac{1}{\pi}\right) \frac{\frac{1}{2} \Delta \lambda_{F W H M}}{\left(\lambda-\lambda_{c}\right)^{2}+\left(\frac{1}{2} \Delta \lambda_{F W H M}\right)^{2}}
$$

were $\lambda_{c}=\lambda_{0} \pm n \cdot \Delta \lambda_{F S R}$ (with $\mathrm{n}$ an integer). The center peak occurs at $\lambda_{c}=\lambda_{0}$ and the side peak occurs at $\lambda_{s p}$. The power transmitted through both rings is now described by the product of the Lorentzian transmission functions of the two individual MRRs at $\lambda_{c}=\lambda_{0}$ and the power of the side peaks can be approximated by the product of the Lorentzian functions $L\left(\lambda_{s p}\right)$ with $\lambda_{c}=\lambda_{0} \pm \Delta \lambda_{F S R, 1}$. Assuming equal coupling coefficients, $\kappa$, for the MRRs, the vale of the PHR, $\delta_{P H R}$, is obtained as 


$$
\delta_{P H R}=\frac{L\left(\lambda_{s p}\right)^{2}}{L\left(\lambda_{c}\right)^{2}}
$$

The meaning of this expression can be seen by inserting Eqs.(2.6,2.7). Given a fixed length of the micro ring resonators, maximizing the PHR, i.e., to maximize the suppression of the feedback of the strongest side peak, is achieved by minimizing the roundtrip losses via decreasing the coupling coefficient $\kappa$.

Summarizing the external cavity design, with Eqs.(2.5-2.8) an MRR mirror can be designed to obtain desired values for free spectral range, the spectral bandwidth and the peak height ratio, $\Delta \lambda_{F S R \text {, tot }}, \Delta \lambda_{F W H M \text {,tot }}$ and $\delta_{P H R}$. An MRR mirror that provides a sufficiently large free spectral range, a small spectral bandwidth, and a low peak height ratio, should result in a narrow bandwidth laser, operating at a single-longitudinal mode, with a high SMSR, and a wide tuning range.

\subsubsection{Laser bandwidth}

The output of lasers is always, to a very small but finite extent, unstable. This is in the large majority of cases caused by effects which can be summarized as technical noise. Examples are, for an external cavity diode laser, electric fluctuations of the pump current or small acoustically induced fluctuations of the length of the resonator. These perturbations cause amplitude and phase noise in the generated light wave. Owing to the wide range of statistical and spectral properties of the perturbations there are several different ways to characterize the resulting noise [29]. The most straightforward and standard way to quantify noise in lasers is to measure what is commonly called the FWHM spectral width of the optical power density, the laser bandwidth or laser linewidth. For lasers with larger bandwidth, such measurements can be performed with optical spectrum analyzers (OSAs) which are, essentially, grating monochromators. Extremely narrow laser bandwidths, however, usually require beat experiments (heterodyne measurements) using a reference laser or self-heterodyning. The spectral broadening of lasers by technical noise can, at least principally, be fully avoided with appropriate passive and active means. Using a low-noise power supply, acoustic shielding or an active stabilization of the cavity length are typical examples.

There is, however, also a fundamental contribution to the laser linewidth, which is intrinsic to the working of a laser and cannot be removed. This contribution comes from spontaneous emission into the resonator modes. For judging the smallest bandwidth that a laser ultimately can provide, it is essential to look a the fundamental bandwidth limit of lasers. This fundamental limit was discussed even before the first experimental demonstration of the laser. The reason is that, in contrast to microwave oscillators, an optical oscillator working at a many orders higher frequency was expected to offer an extremely low bandwidth. This expectation was quantified for the first time in 1958, when 
Schawlow and Townes wrote their famous article on the fundamental bandwidth limit of lasers, which back then they tentatively called "infrared and optical masers" [30]. Considering a symmetric two mirror Fabry Perot type of cavity with weak output coupling, they calculated the lower limit of the frequency bandwidth, $\Delta \nu_{S T}$, as ${ }^{2}$

$$
\Delta \nu_{S T}=\frac{\pi h \nu\left(\Delta \nu_{c}\right)^{2}}{P},
$$

where $h$ is Planck's constant, $\nu$ is the frequency of the laser's output, and $P$ is the output power of the laser. Eq.(2.9) is since known as the SchawlowTownes or quantum limited bandwidth of a laser. The FWHM cavity bandwidth, $\Delta \nu_{c}$, is given by the cavity parameters,

$$
\Delta \nu_{c}=\frac{-c \cdot \ln \left(R_{f f} R_{b f}\right)}{4 \pi n_{d} L_{d}}
$$

where the reflectivity of the two mirrors, respectively, is $R_{f f}$ and $R_{b f}$ and the cavity length is $L_{d}$ and where $n_{d}$ is the index of the laser material that fills the space between the two mirrors. Sometimes it is more convenient to use a bandwidth in the wavelength range rather than the frequency range. This is done by rewriting Eq.(2.10) to

$$
\Delta \lambda_{F W H M, d}=\frac{-\lambda_{0}^{2} \cdot \ln \left(R_{f f} R_{b f}\right)}{4 \pi n_{d} L_{d}} .
$$

An important step in determining methods to decrease the laser bandwidth is to describe, next to the cavity bandwidth, the other optical properties of a cavity. Of significant importance are the free spectral range, the finesse and the Q-factor. These properties can, for the diode lasers used in this research, approximated by those of a Fabry Perot type optical cavity. Similar to Eq.(2.5), which describes the free spectral range of an MRR, for an optical Fabry Perot type cavity this is described as

$$
\Delta \lambda_{F S R, d}=\frac{\lambda_{0}^{2}}{2 n_{d} L_{d}} .
$$

The finesse $F$ of an optical cavity is described by [31]

$$
F=\frac{\Delta \lambda_{F S R}}{\Delta \lambda_{F W H M}}
$$

and the Q-factor of an optical cavity is given as [31]

$$
Q=\frac{\lambda_{0}}{\Delta \lambda_{F W H M}}
$$

\footnotetext{
${ }^{2}$ The original equation denoted the bandwidth in half width at half maximum, Eq.(2.9) is the adaptation for full width at half maximum (FWHM).
} 
To give an example, the diode laser in our work has a free spectral range of $0.95 \mathrm{~nm}$ and a spectral bandwidth of its modes of $2.75 \mathrm{~nm}$ (values for $n_{d}$, $L_{d}, R_{f f}$ and $R_{b f}$ are provided in Section 2.3), which yields a finesse of 0.35 . Using the values that are derived for our MRR design $\left(\Delta \lambda_{F S R, t o t}=44 \mathrm{~nm}\right.$ and $\Delta \lambda_{F W H M, t o t}=0.5 \mathrm{~nm}$ ), we calculate a finesse of $F=88$, using Eq.(2.13).

Although Eq.(2.9) is just a good estimate based on thermodynamic arguments and assuming a cavity with a specific format, the equation proved to describe the bandwidth for many types of lasers quite well, until measuring the bandwidth of diode lasers. The experiments showed that even with low technical noise, the lower limit as described by Eq.(2.9) could clearly not be achieved. It was later shown by Henry [19] that the excess spectral bandwidth of diode lasers could be attributed to the coupling of index variations to gain fluctuations. Thereby, the spontaneous emission events in diode lasers do not only lead directly to phase and amplitude fluctuations. The spontaneous emission events also reduce the laser inversion and gain. Also the subsequent recovery of the laser to steady state intensity results in changes in the refractive index, which let the optical cavity length fluctuate. This causes additional phase fluctuations and thus a spectral broadening. The corresponding enhancement of the bandwidth grows with the rate of the index change, that the semiconductor gain material exhibits for a given change of the gain. This ratio, $\alpha=\Delta n^{\prime} / \Delta n^{\prime \prime}$ (where $n^{\prime}$ and $n^{\prime \prime}$ are the gain and index expressed as the real and imaginary parts of the complex index of refraction) is a material property, called the linewidth enhancement factor. Recalculating the Schawlow-Townes limit for diode lasers, Ref. [19] shows that the linewidth increases by a factor of $1+\alpha^{2}$,

$$
\Delta \nu_{S T, D L}=\frac{\pi h \nu\left(\Delta \nu_{c}\right)^{2}\left(1+\alpha^{2}\right)}{P} .
$$

The bandwidth given in Eq.(2.15) is still derived for a solitary diode laser, e.g., the free running Fabry Perot laser. However, Eq.(2.15) also indicates how further reduction can be achieved. Particularly effective is to equip the diode laser with an external cavity which elongates the cavity length $L_{d}$. Increasing the cavity length via Eq.(2.10) enters quadratically as linewidth reduction.

An additional narrowing can be achieved when adding a high-Q cavity, such as an MRR, to the external cavity. Laurent et al. [32] calculated the decreased bandwidth using rate equations for the temporal evolution of the laser field. They show that reduction of the bandwidth of the external cavity semiconductor laser, $\Delta \nu_{E C S L}$, can be described as a further quadratic factor,

$$
\Delta \nu_{E C S L}=\frac{\Delta \nu_{S T, F R}}{\beta\left(\frac{n_{e c} L_{e c}}{n_{d} L_{d}} \cdot \frac{F_{e c}}{F_{d}}\right)^{2}},
$$

where $n_{e c}$ is the refractive index in the optical path of the external cavity, $L_{e c}$ is the physical length of the external cavity, $n_{d}$ is the effective refractive index of the diode laser, $L_{d}$ is the physical length of the diode laser, $F_{e c}$ is the 
finesse of the external cavity and $F_{d}$ is the finesse of the diode laser cavity. $\beta$ accounts for the losses in the cavity $(\beta=0$ means all intracavity light is lost, $\beta=1$ means no intracavity losses). The laser bandwidth, $\Delta \nu_{S T, F R}$, as used in Eq.(2.16) by Laurent et al., is the free running diode laser that would be seen without the external feedback and without the linewidth enhancement caused via $\alpha$. This bandwidth can be obtained from a measurement of the free running diode bandwidth, $\Delta \nu_{F R}$, and the relation $\Delta \nu_{S T, F R}=\Delta \nu_{F R} /\left(1+\alpha^{2}\right)$.

For an evaluation of Eq.(2.16), to enable a comparison with experimental data in Chapter 3, all of the latter named parameters of the diode and the external cavity need to be determined. The measured bandwidth for an antireflection coated diode laser will typically be in the order of several GHz. The finesse will be below one. The finesse of the MRR mirror is expected to be larger than 100 for certain design parameters. Based on these values, an estimate of the expected optical bandwidth of the WECSL is given after the WECSL design is discussed, at the end of Section 2.3.

To conclude, for the special MRR mirror to be used in our experiments, where two MRRs are to be passed in series, the FSR is significantly increased via the Vernier effect. The bandwidth of the center peak of the reflected light $\left(\Delta \lambda_{F W H M, t o t}\right)$ is described by Eq.(2.6) and Eq.(2.7). It can be seen that the bandwidth of the WECSL decreases with a decreasing bandwidth of the MRR mirror and increasing free spectral range. The bandwidth can be lowered by decreasing the MRR coupling coefficient $\kappa$. Also the free spectral range can be increased by decreasing the difference between the two MRR radii. It should however be noted that decreasing $\kappa$ also decreases the amount of feedback and thereby requires a low reflectivity of the diode laser facet (see Eq.(2.4)). A decrease in the difference of the MRR's radii also decreases the PHR (see Eq.(2.8)). However a minimum PHR is required for the diode laser to operate on a single frequency. In summary, to design a WECSL with low spectral bandwidth, the FWHM can be decreased and the FSR increased, as long as the MRR mirror provides the proper feedback for single frequency operation.

\subsection{WECSL design}

In view of the broad availability of AR-coated diode lasers at telecommunication wavelength ranges, we chose to design a WECSL that operates at a telecommunication band (C-band from $1530 \mathrm{~nm}$ to $1565 \mathrm{~nm}$ ). This WECSL is fabricated and the experimental results are shown in Chapter 3. To be able to tune the WECSL over the full C-band, it is required that the free spectral range of the MRR mirror is $>35 \mathrm{~nm}$.

The selected laser diode is a custom anti-reflection coated angled-stripe gain chip (Fraunhofer Heinrich-Hertz-Institut). A schematic representation of the chip is shown in Fig. 2.4. The typical specified reflection of the back facet is $0.85 \pm 0.1$. The AR coating of the angled front facet has a specified maximum reflectivity of $10^{-3}$. The diode laser's beam radius at the facet of both axes 
$w_{d, x}$ and $w_{d, y}$ is $3 \mu \mathrm{m}$. At the output facet, the gain waveguide forms an angle of $\varphi=5^{\circ}$ with respect to the facet normal. Multiplying the residual reflectivity of the AR with that calculated by Eq.(2.2), the total reflection of the front facet back into the laser is calculated to be reduced to $1.46 \cdot 10^{-8}$. For the estimated linewidth-enhancement factor of $\alpha=5$, this means the required amount of feedback into the diode laser would be $3 \cdot 65 \cdot 10^{-7}$ (Eq.(2.4)). The amount of required feedback will increase when a waveguide is butt-coupled to the diode laser, because of the reflection on the waveguide facet. The required feedback can be calculated using Eq.(2.1), Eq.(2.2) and Eq.(2.4). For a refractive index of the waveguide of $n_{g}=1.73$ (this value will be explained below), it means that the required amount of feedback increases to $2.6 \cdot 10^{-5}$.

a)

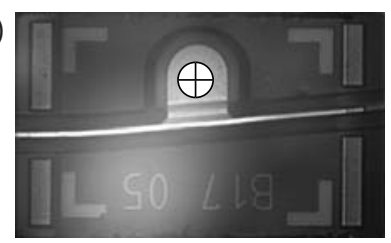

b)

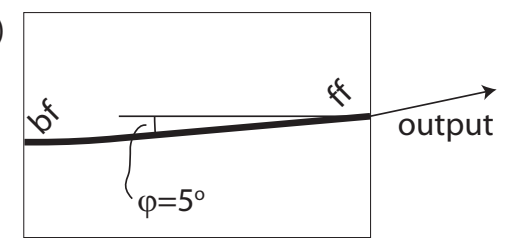

Figure 2.4: Top view microscope photograph (a) and schematic (b) of the anti-reflection coated angled-stripe gain chip. In (b) the electrical contacts are omitted. The thick black line in (b) represents the waveguide of the diode laser where gain is generated, ff indicates the anti-reflection coated front facet, bf the highly reflective back facet, $\varphi$ indicates the angle of the laser waveguide normal to the front facet $\left(5^{\circ}\right)$. $\oplus$ indicates the electrical contact of the positive electrode, whereas the negative electrode is on the bottom side (not seen in figure).

The frequency selective waveguide circuits are designed, based on filtering by two subsequent MRRs, using $\mathrm{Si}_{3} \mathrm{~N}_{4} / \mathrm{SiO}_{2}$ waveguide technology with a boxshaped cross section. The choice for this technology is motivated by the goal to provide frequency selective feedback with low losses, as this would provide a narrow laser bandwidth. Indeed, the losses associated with the used waveguide technology are low, i.e., low waveguide scattering losses $(\leq 0.06 \mathrm{~dB} / \mathrm{cm})$ and a relatively high index contrast $(\Delta \mathrm{n}=0.1-0.5)$ yielding low radiative bending losses $(\sim 1 \mathrm{~dB} / \mathrm{cm}$ for a bend with a radius of $50 \mu \mathrm{m})[22,33]$. To provide a large wavelength tuning range to the WECSL, a large FSR is required for the MRR, which is achieved by a small MRR radius. A radius of $\mathrm{R}=50 \mu \mathrm{m}$ for MRRs is the smallest radius that can be fabricated reproducibly, with acceptable radiative bending losses (we chose $1 \mathrm{~dB} / \mathrm{cm}$ to be acceptable, because bending losses $<1 \mathrm{~dB} / \mathrm{cm}$ can be safely neglected with respect to in- and output coupling). With the design values of $n_{g}=1.73$ and $R_{1}=50 \mu \mathrm{m}$, the expected free spectral range is $4.4 \mathrm{~nm}$ at $\lambda_{0}=1550 \mathrm{~nm}$. To compensate for possible deviations in the FSR by fabrication errors, we chose to have the FSRs differ by $10 \%$, such that $\Delta \lambda_{F S R, 2}=4.0 \mathrm{~nm}$ and $\Delta \lambda_{F S R, t o t}=44 \mathrm{~nm}$, which renders a design diameter of 
the second MRR of $R_{2}=55 \mu \mathrm{m}$. The layout of the frequency selective mirror, consisting of Y-splitters, directional and bi-directional couplers and two MRRs, is shown in Fig. 2.5. To optimize coupling between the diode laser and the external waveguide cavity, the input of the waveguide is oriented at an angle $\left(\psi=9.11^{\circ}\right)$ with respect to the normal of the waveguide chip facet. This angle takes into account the difference in refractive indices of the interface between higher index gain chip $\left(n_{d}=3.16\right)$ and the lower effective index of the waveguide $\left(n_{e c}=1.73\right)$. The bi-directional symmetric coupler ( $C$ in Fig. 2.5) taps off $\sim 5 \%$ of the light into what we call measurement channels. This feature was included to allow the laser and mirror performance to be monitored in more detail. It allows us to monitor the output of the diode laser, without disturbing the laser's output channel.

So far we have only considered a perfect coupling between diode laser and waveguide. However, if the beam radii at the facets of the waveguide and the diode laser are not equal, this can cause considerable losses $\Gamma$ in coupling light from the diode laser into the waveguide and back. The accordingly reduced transmission, $T$, can be calculated by evaluating a mode overlap integral, yielding $[23]$ as

$$
T=\frac{4}{\left(\frac{w_{d, x}}{w_{w g, x}}+\frac{w_{w g, x}}{w_{d, x}}\right) \cdot\left(\frac{w_{d, y}}{w_{w g, y}}+\frac{w_{w g, y}}{w_{d, y}}\right)}
$$

with $T$ the power transmission (i.e., $T=1-\Gamma$ ). For our diode laser $\left(w_{d, x}=w_{d, y}=3 \mu \mathrm{m}\right)$ and waveguide $\left(w_{w g, x}=0.45 \mu \mathrm{m}, w_{w g, y}=0.65 \mu \mathrm{m}\right)$ we calculate $T=0.12$. This limits the maximum feedback from the MRR mirror into the diode laser to a value of about 1\%. According to Eq.(2.4) the diode laser receives the required feedback when the MRR mirror reflects at least $2.1 \cdot 10^{-3}$. This amount is calculated using Eq.(2.1), Eq.(2.2) and Eq.(2.17) and includes all losses within the waveguide chip, such as waveguide scattering losses, bending losses, coupling losses and absorption. 

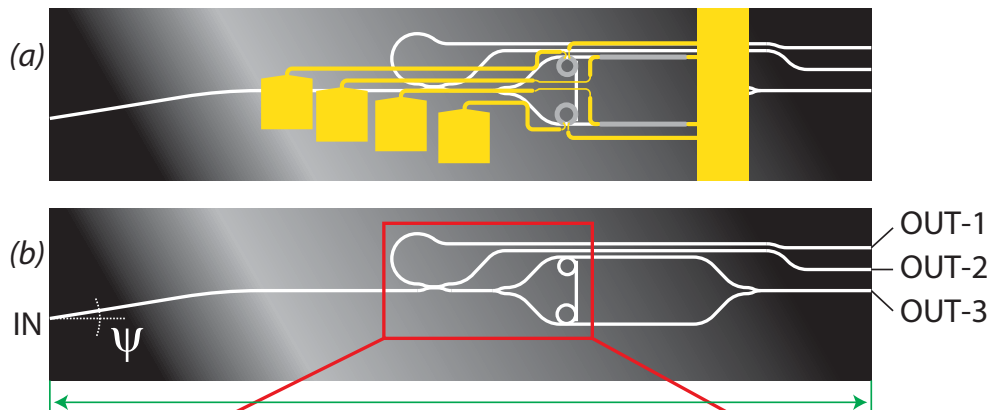

(c)

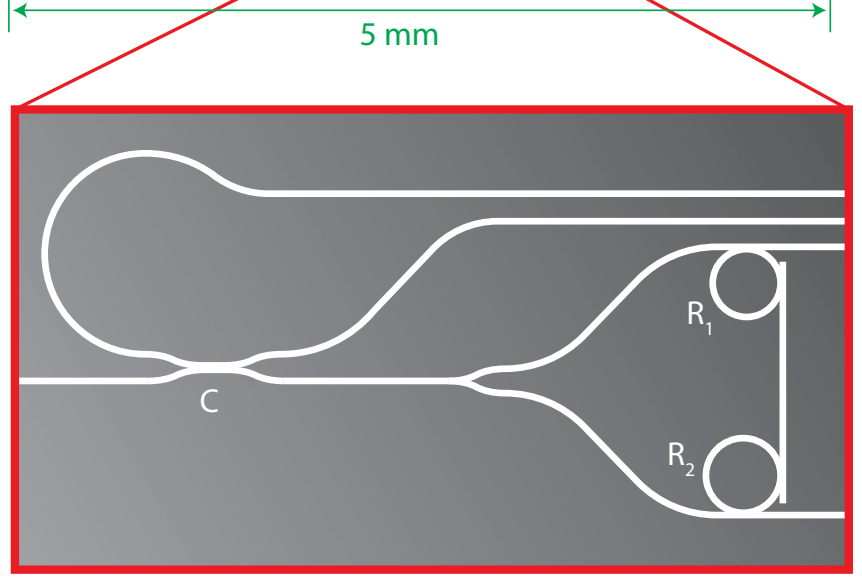

Figure 2.5: Schematic of the waveguide chip, to scale. (a) shows the complete waveguide chip. The waveguides are depicted in white, electrical contacts are in yellow, and heaters are in gray. The heaters are placed on top of the MRRs (for thermal tuning of the MRRs' resonance frequencies), and on the straight waveguides after the MRR mirror (for changing the refractive index of the straight waveguides after the MRRs, such that a maximum output power can be achieved after the two straights are combined). (b) shows only the waveguides. $\psi$ indicates the angle of the waveguide normal to the facet $\left(9.11^{\circ}\right)$. (c) is a zoom-in on the two MRRs, having radii $R_{1}$ and $R_{2}$, and the coupler $C$.

Since the peak-height ratio (PHR) devided by the main peak to side-peak spacing needs to be smaller than the steepest slope in the gain profile of the laser, a sufficiently small PHR needs to be provided by design. The steepest slope of the gain spectrum of the laser gain chip used in this research is $0.48 \mathrm{~dB} / \mathrm{nm}$, requiring that the peak heigth ratio is smaller than 0.63 (i.e., more than $2.0 \mathrm{~dB}$ over the wavelength range $\left.\lambda_{0}-\lambda_{s p}\right)$. Combining Eqs.(2.7 and 2.8), with the parameters determined earlier $\left(R_{1}=50 \mu \mathrm{m}, R_{2}=55 \mu \mathrm{m}\right.$, and $\left.n_{g}=1.73\right)$ shows that the spectral bandwidth $\Delta \lambda_{F W H M, t o t}=0.52 \mathrm{~nm}$, requiring the coupling coefficient $\kappa^{2}<0.44$. 
To validate these results, we have calculated the values for the free spectral range, bandwidth, and peak height ratio in more detail, using the general method, as described by Yariv [25, 34], or to more detail in the PhD Thesis of Klein [35]. These calculations resulted in a set of optimized parameters in agreement with those found above $\left(\Delta \lambda_{F S R, t o t}=44 \mathrm{~nm}, \delta_{P H R}=0.63\right.$, and $\Delta \lambda_{F W H M, t o t}=0.51 \mathrm{~nm}$, for $\left.\kappa^{2}=0.44\right)$. The calculated reflectivity spectrum, taking ring radii $\mathrm{R}_{1}=50 \mu \mathrm{m}, \mathrm{R}_{2}=55 \mu \mathrm{m}$ and group index $\mathrm{n}_{g}=1.73$, is shown in Fig. 2.6.

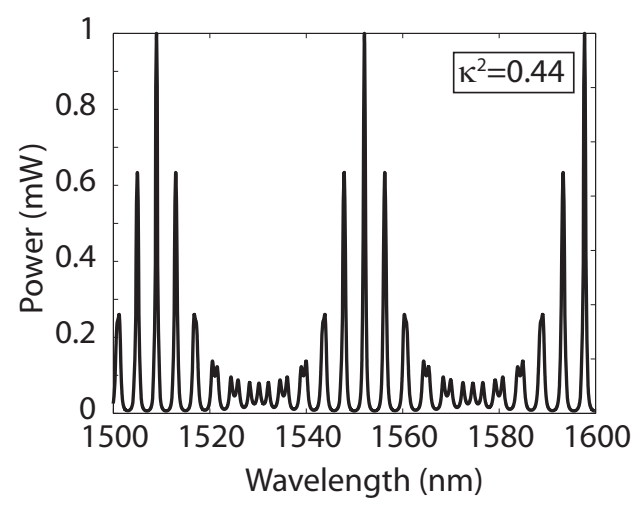

Figure 2.6: Calculated reflectance spectrum of the MRR mirror, using $\kappa^{2}=0.44, R_{1}=50 \mu \mathrm{m}, R_{2}=55 \mu \mathrm{m}$ and $n_{g}=1$.73. The calculations did not take any additional losses into account.

Due to many different steps in fabrication, it is challenging to fabricate -purely by design- an MRR mirror with the exact coupling coefficient of $\kappa^{2}=0.44$. In practice, the intended coupling coefficient is expected to possess a fabrication error of at least $\kappa^{2} \pm 0.05$. To secure a suitable value of $\kappa$, we fabricated a set of MRR mirrors in which the nominal design value of $\kappa$ is systematically varied in steps smaller than the fabrication error. We have designed the MRR mirrors such that the values for $\kappa$ cover at least $0.3<\kappa^{2}<0.4$. Thereby we ensure that an MRR mirror with a peak height ratio $<0.63$ can be obtained. In addition, this makes MRR mirrors available with a variety of $\kappa$ values, which is of interest for investigating variations in the performance of MRR mirrors.

Giving an estimate for the expected values of the optical bandwidth and Q-factor for such complex cavity designs is not straight forward. These values depend depends heavily on measured values, since small fabrication errors can have a profound effect on certain parameters of the waveguide design. For example, a small difference in waveguide width will change the MRR's coupling coefficient and effective roundtrip length. This will affect the finesse of the MRR mirror and thus the expected Q-factor of the laser cavity and the bandwidth of the laser. However, to give a rough estimate, we use the following values. 
First we need to calculate a finesses of the external cavity and the diode laser. We start with the MRR mirror's MRR radii $\mathrm{R}_{1}=50 \mu \mathrm{m}$ and $\mathrm{R}_{2}=55 \mu \mathrm{m}$, both with coupling coefficient $\kappa^{2}=0.4$ and a group index of $\mathrm{n}_{g}=1.73$. This gives via Eq.(2.6) and Eq.(2.7) an MRR mirror bandwidth of $\Delta \lambda_{F W H M, t o t}=0.45 \mathrm{~nm}$ and via the explanation below Eq.(2.5) a free spectral range of $\Delta \lambda_{F S R, t o t}=$ $44 \mathrm{~nm}$. Using Eq.(2.13) we finally come to the finesse of the MRR mirror $F_{e c}=97$. The used solitary diode laser has a finesse of 0.35 (see calculation below Eq.(2.11)). The loss between diode laser the MRR mirror is estimated in the next chapter to provide a value for the overall feedback efficiency of $\beta=2.5 \cdot 10^{-3}$ to be used in Eq.(2.16). The effective optical length of the diode laser is $1.3 \mathrm{~mm}$, the effective optical length of the external cavity is $6.8 \mathrm{~mm}$. We use a bandwidth of $\Delta \nu_{S T, F R}=96 \mathrm{MHz}$ which we derive from measurements in the next chapter. Eq.(2.16) then provides an estimated bandwidth of $18 \mathrm{kHz}$ for the output of the WECSL. Improvements in alignment and tapering to match the beam radii of the diode laser and the waveguide at their respective facets can drastically decrease the lower fundamental bandwidth. For example, if we assume $\beta=1$, using Eq.(2.16) we calculate a lower bandwidth limit of $7.5 \mathrm{~Hz}$.

Deriving the Q-factor of the WECSL is also not straight forward. Because of the complex cavity layout, we cannot simply calculate the cavity ringdown time. However, if we assume tentatively that the WECSL resonator can be modeled as an effective Fabry Perot resonator, we can use Eq.(2.15) to calculate the cavity bandwidth, based on the estimated Schawlow-Townes bandwidth of $18 \mathrm{kHz}$, for which we have assumed an output power of $1 \mathrm{~mW}$ (similar to the experimental value, see Chapter 3). Subsequently, we can use this cavity bandwidth to calculate the Q-factor, using Eq.(2.14). This leads to a Q-factor of $1.5 \cdot 10^{5}$. In Chapter 3, Section 3.4.2, we come to a somewhat lower Q-factor of $7.7 \cdot 10^{4}$. This can be explained by the lower output power $(0.6 \mathrm{~mW})$ and by small deviations and misalignments of the WECSL that would lead to a broader bandwidth. In this case, if the Q-factor indeed was lowered by misalignment to a value of $7.7 \cdot 10^{4}$, the expected laser bandwidth would be $114 \mathrm{kHz}$. This shows that small deviations in alignment and fabrication can have a profound effect on the spectral behavior of the WECSL.

\subsection{Injection locking}

The previous section considered how to control the frequency and spectral bandwidth of a diode laser oscillator via external feedback using a waveguide circuit. An extended spectral control of laser oscillators can be achieved via socalled injection locking $[13,36,37]$. This technique enables to externally control the optical phase of a laser oscillator by injecting light from another laser even if the injected laser has a much lower power. In case of successful injection locking the two oscillators will oscillate in phase at an identical frequency. The advantage compared to simple amplification is that injection locking allows for 
higher signal to noise ratios and also introduces a strong spectral selectivity [37]. When performing injection locking with multiple oscillators in parallel, all of these will oscillate in phase, which is of interest for providing phased arrays of lasers in certain applications.

A potential application of immediate relevance is microwave communication with phased antenna arrays (PAA). Each single antenna element (AE) in the array receives microwaves from a wide solid angle. However if the received signals are properly delayed with regard to each other and then superimposed, the resulting signal is equivalent to what would have been received by a large antenna with high directionality. While delaying microwave signals directly requires bulky equipment, a most elegant approach is to use optical delay lines. For this approach, the received microwave signals are used to modulate an optical carrier, thereby transforming the received information to the optical domain. The signals received by the antenna elements can be properly delayed with respect to each other in the optical domain, thereby forming a so-called optical beam forming network (OBFN) [38]. The advantage of optical delay techniques is that these can be implemented with dramatically reduced size, compared to microwave delay lines, which is due to the approximately 5 orders of magnitude smaller wavelength of light compared to microwaves. This is illustrated, e.g., in a recent publication by Meijerink et al. [2] which discusses the implementation of 16 independent delay lines in optical waveguides with a total physical size of only a few $\mathrm{mm}$.

One of the requirements of such on-chip miniaturized solutions for phased array antennas is that the signals received by each antenna element are separately modulated onto a suitable set of optical carrier frequencies, the relative optical phases of which are controlled. If separate lasers for each AE were used without phase controlling them, this would lead to an uncontrolled superposition of their output waves within a dephasing time, which is on the order of the inverse laser band width. A possible solution, providing the required set of well-phased optical waves is to realize an entire array of WECSLs, next to each other on the same waveguide chip and use on-chip injection locking to control the optical phases throughout the entire array of oscillators.

To investigate the potential of such applications we have investigated the corresponding prototype scenario, which is injection locking of a single WECSL by another WECSL.

\subsubsection{Injection locking principle}

For laser oscillators, such locking is performed by injecting the light of a first laser, called the master laser, into a second laser, called the slave laser [13]. Consider a master laser with output power $P_{i n}$ and an optical frequency $\nu_{m}$, and an unlocked slave laser with an optical frequency $\nu_{s}$ and output power $P_{\text {out }}$. If the frequencies of the master and slave laser are within a certain range from each other, the relative phase of the slave is locked to that of the master 
and the frequency of the slave laser becomes equal to that of the master laser, such that $\nu_{s}=\nu_{m}$ [37]. This is called injection locking. The frequency range over which locking can be observed is called the locking range $\Delta \nu_{\text {lock}}$, i.e., $\nu_{m}=\nu_{s} \pm \frac{1}{2} \Delta \nu_{\text {lock }}$.

The relation to describe the locking range $\Delta \nu_{l o c k}$, as given by Adler [36], modified to describe the locking range of the slave laser gives:

$$
\Delta \nu_{\text {lock }}=\frac{\nu_{s}}{Q} \sqrt{\frac{P_{\text {in }}}{P_{\text {out }}}},
$$

where $Q$ is $Q$-factor of the slave laser's resonator. Eq.(2.18) shows that the locking range is determined by the specific optical and operational parameters of the laser to be locked, but also by the amount of injected light from the master oscillator $\left(P_{i n}\right)$. In situations such as the above mentioned microwave photonic applications, it can be required to employ injection locking for a frequency selective amplification of an optical sideband. This requires that the locking range comprises the full RF sideband of the received microwave, which has a width in the order of several hundred $\mathrm{MHz}$. If the locking range were considerably smaller, only part of the RF sideband would be amplified. To avoid this, according to Eq.(2.18), the locking range can be increased theoretically via increasing the power of the injected sideband. However, as the available sideband power is usually low, possibly in the order of micro Watts, it is important to obtain quantitative experimental information about the locking range and confirm its variation with the injected power.

The injection locking range of a laser can be measured by two different methods. During sweeping of the master frequency across the range surrounding the slave frequency, one can observe the beat frequency of the two lasers by superimposing their output on a detector. Successful locking is observed as the disappearance of the beat signal from the detector output. The locking range can be determined by recording over what range of the swept frequency the beat signal disappears.

In our experiments we have used a setup which is very similar to that described by Stover and Steier [13], as shown in Fig. 2.7. Light from the slave laser does not influence the master laser, due to the optical isolator. The output light of both master and slave laser are collected on a photodiode. The beat signal of the frequencies of the master and slave laser is displayed on an RF spectrum analyzer. The results of our experiments are discussed in Chapter 3, Section 3.4.2.

To give an estimation of the expected locking range, we can use the Qfactors described in Section 2.3. Using values for the measured output power and input power derived in Chapter 3, Section 3.4.2, $\left(P_{\text {out }}=0.6 \mathrm{~mW}\right.$ and $P_{\text {in }}=1.5 \cdot 10^{-2} \mathrm{~mW}$ ), we calculate an expected locking range using Eq.(2.18). For $\mathrm{Q}=1.5 \cdot 10^{5}$, we estimate the locking range to be $205 \mathrm{MHz}$ and for $\mathrm{Q}=7.7 \cdot 10^{4}$ we estimate a locking range of $400 \mathrm{MHz}$. 


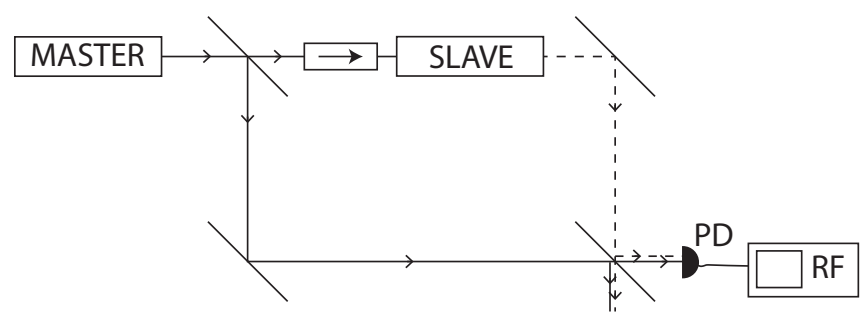

Figure 2.7: Schematic of the injection locking setup. Light from the master laser passes through a beam splitter, the light in one arm passes through an optical isolator and is injected into the slave laser. The light is combined by a second beam splitter and superimposed onto a fast photodiode, which is connected to an RF spectrum analyzer.

For completeness, a second method [39] to determine the injection locking range is shortly described below. However, it should be noted that for our experiments this second method proved to have a too large readout error ${ }^{3}$. Therefore only the first, more accurate, method was used. The second method is described as follows. To determine the injection locking range, the intensity of the RF-beat spectrum is examined when the frequency of the master laser is just outside of the locking range of the slave laser. The slave laser behaves as an unlocked oscillator driven into frequency modulation at the lasers' difference frequency. This generates a non-symmetric sideband distribution about the carrier, with sidebands mainly on one spectral side of the driving frequency. From the sidebands' amplitude and frequency spacing the locking range can be determined. For an extensive explanation of this method, the reader is referred to the article by Stover [39].

\subsection{Conclusions}

In this chapter we have analyzed some essential requirements to realize single frequency operation with a waveguide based external cavity semiconductor laser (WECSL). We have investigated the required properties for the two main components that comprise the WECSL, which are the semiconductor gain chip and the frequency selective waveguide circuit that provides the external feedback. The specific implementation of a highly frequency selective feedback via two micro ring resonators (MRRs), based on available technology has been described in more detail. The values for the design parameters in this implementation indicate that it is possible to obtain single frequency oscillation across the entire telecommunication C-band (1530-1565 nm).

\footnotetext{
${ }^{3}$ The readout error of the first method only comprises of an error in the RF frequency, in our experiment about $50 \mathrm{MHz}$. The second method has a readout error in the RF frequency and in the RF intensity. The latter is about $2-3 \mathrm{dBm}$ in our experiments, which relates to an error in the locking range of $>500 \mathrm{MHz}$.
} 
Furthermore, we expect a narrow optical bandwidth from the WECSL, well below $1 \mathrm{MHz}$. More specifically, for the envisioned parameters of our specific implementation, i.e., a coupling coefficient of $\kappa^{2}=0.4$ for the two MRRs with radii $R_{1}=50 \mu \mathrm{m}$ and $R_{2}=55 \mu \mathrm{m}$ and a free spectral range of $\Delta \lambda_{F S R, t o t}=44 \mathrm{~nm}$, we estimate the expected laser bandwidth to lie in the order of $18 \mathrm{kHz}$. We expect that reducing the spectral bandwidth of the laser can be done by optimizing the feedback parameters of the micro ring resonators, with the goal to increase the finesse of the MRR mirror. A measurement of the effective finesse of the complex resonator structure of WECSLs after assembly, i.e., including the effects of residual losses at facets, can be done via injection locking. For instance, we have determined the effective finesse of our WECSL resonator by measuring the locking range. Monitored by such measurements, the finesse can be increased by increasing the free spectral range by reducing the feedback bandwidth. This can be done by increasing the finesse of the MRRs themselves, by decreasing the coupling coefficient, $\kappa$, and thereby decreasing the bandwidth of the reflection. However, decreasing the coupling coefficient causes resonant light to travel many more roundtrips through the MRRs before being reflected back to the laser. This increases the overall loss, due to the radiative bending losses and scattering losses inside the MRRs. In summary, the lowest reflected bandwidth for the given waveguide geometry can be achieved by optimizing for the highest finesse by lowest coupling coefficient, while maintaining sufficient feedback for the laser. Other geometries might be worth investigating, such as based on two identical resonators, not in series but mutually coupled, to reduce the feedback bandwidth $[40,41]$. 



\section{3 WECSL experiments}

\subsection{Introduction}

In the previous chapter we presented a set of design considerations for the purpose to realize an external cavity diode laser with unique properties. These are in particular single mode operation, widely tunable at around $1550 \mathrm{~nm}$ wavelength and with an extremely narrow spectral bandwidth in the tens of $\mathrm{kHz}$ range.

Using the design considerations, we arrived at a layout of waveguide circuits in which an entire set of parameters is to be realized simultaneously. These are, recalling from Chapter 2: The radii of the micro-ring resonators $\left(R_{1}=50 \mu \mathrm{m}\right.$ and $R_{2}=55 \mu \mathrm{m}$ ), to yield a free spectral range (FSR) of $\Delta \lambda_{F S R, t o t}=44 \mathrm{~nm}$, and an input-output coupling coefficient, $\kappa^{2}$, for the rings of about 0.35 , to achieve a peak height ratio (PHR) of $\delta_{P H R}$ less than 0.63 . The reflectivity (on-chip) of the MRR mirror has to exceed $2.1 \cdot 10^{-3}$.

For the fabrication of a larger number of waveguide circuits, a corresponding mask for optical lithography was designed [42] and generated with e-beam lithography. Using the mask, the waveguide circuits were fabricated on a wafer in $\mathrm{Si}_{3} \mathrm{~N}_{4} / \mathrm{SiO}_{2}$ waveguide technology (TriPleX ${ }^{T M}$ ) [22] at LioniX B.V. [43] in collaboration with XiO Photonics B.V. [44] using the Nanolab facilities of the MESA+ institute for nanotechnology [45]. The wafer was diced into separate chips, followed by polishing of the input and output facets which was essential for a proper working and characterization.

In order to ensure that fabrication would lead to parameters close to the design values in spite of inherent fabrication errors (typically, e.g., a deviation of $50 \mathrm{~nm}$ in the width of the waveguides), all circuits are fabricated multiple times, but with slightly varying parameters. Specifically the MRR mirrors were 
fabricated with an entire range of design values for the coupling coefficients $\kappa^{2}$ between 0.3 and 0.4 at least with several intermediate values. This range corresponds to a range in bandwidth of the light reflected by a double MRR mirror circuit, $\Delta \lambda_{F W H M, t o t}$, from 0.2 to $0.5 \mathrm{~nm}$.

After fabrication a set of 10 different MRR mirror circuits were identified and further characterized in optical measurements. Indeed, several of these MRR mirrors showed a free spectral range of about $44 \mathrm{~nm}$, combined with a peak height ratio, $\delta_{P H R}$, and spectral width, $\Delta \lambda_{F W H M, t o t}$, that correspond to a coupling parameter, $\kappa^{2}$, between 0.3 and 0.4 . Out of this set, we chose the MRR mirror with the highest throughput (measured from port IN to port OUT-3, as indicated in Fig. 3.1), for a more detailed qualification. The characterization is presented in the first part of this chapter, Section 3.3.

To compare the spectral properties of the diode laser with and without MRR mirror, in Section 3.3 measurements on the basic properties of the free running diode laser (without MRR mirror) are presented, which comprise a determination of the laser threshold, maximum output power, side-mode suppression ratio (SMSR) and the spectral bandwidth.

Section 3.4 presents the optical characterization of the WECSL, i.e., the diode laser controlled by feedback from the waveguide MRR mirror. In a first step we characterize the WECSL in terms of its laser threshold, maximum output power, SMSR, tuning range and the maximum speed of switching access between two arbitrarily chosen wavelengths. In a second step, special attention is paid to measurements regarding the spectral bandwidth of the WECSL. In addition, to investigate also a control of the optical phase of light generated by a WECSL, we present experimental results in Section 2.4 on the injection locking with the output from a second WECSL. 


\subsection{Characterization of waveguide circuits}

IN

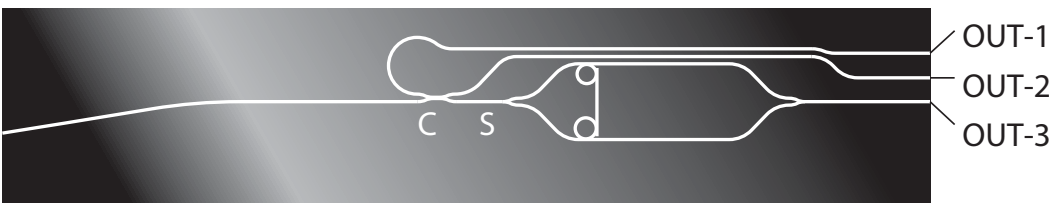

$1 \mathrm{~mm}$

Figure 3.1: Schematic of the waveguide chip to scale, as presented in Chapter 2, Fig. 2.5b. OUT-1 and OUT-2 indicate the output ports of the measurement channel and OUT-3 indicates the output port of the WECSL. Point $\mathrm{S}$ is the internal reference point with regard to which measurements at ports OUT-1 and OUT-2 yield the internal reflectivity of the pair of ring resonators. Point $\mathrm{C}$ designates the location of the broadband waveguide coupler which diverts about 5\% of the input light (from IN) to the measurement channel OUT-2, and which diverts also about 5\% of the light reflected by the MRRs to the measurement channel OUT-1.

The characterization began with a set of ten MRR mirrors that presumably have their coupling coefficients in the appropriate range. Among these, the next selection was based on throughput measurements. Light from a fiber coupled super luminescent diode (SLD) (Thorlabs S5FC1005S) was butt-coupled via a PM-fiber to the input port of the waveguide chip (Fig. 3.1, IN). The throughput power was measured at the other side of the chip $(O U T-3)$ via coupling to a single-mode fiber, to a calibrated fiber-coupled power meter (HP81536A). The spectrally dependent reflectivity of the MRR mirror was measured with an optical spectrum analyzer (OSA) (ANDO AQ6317). We found four MRR mirrors with a measured peak height ratio lower than 0.6. For characterization in more detail out of this set of MRR mirrors, we selected the MRR mirror that showed the highest throughput power.

Spectral measurements were performed using a single-mode fiber connection to the input of the OSA to ensure maximum spectral resolution. The OSA has a minimum resolution of $0.01 \mathrm{~nm}$, which is about $1.5 \mathrm{GHz}$ in the wavelength range used here, around $1550 \mathrm{~nm}$. The spectrum of the input light from the SLD used to further inspect the selected MRR mirror, is shown in the inset in Fig. 3.2, normalized to the maximum spectral power density. The spectrum of the light reflected by the MRR mirror was measured at the output port of the measurement channel OUT-1 (Fig. 3.1). The power reflected by the MRR mirror vs. wavelength, recorded over a range much wider than the telecom C-band (1530-1565 nm) is displayed as the main graph in Fig. 3.2. 


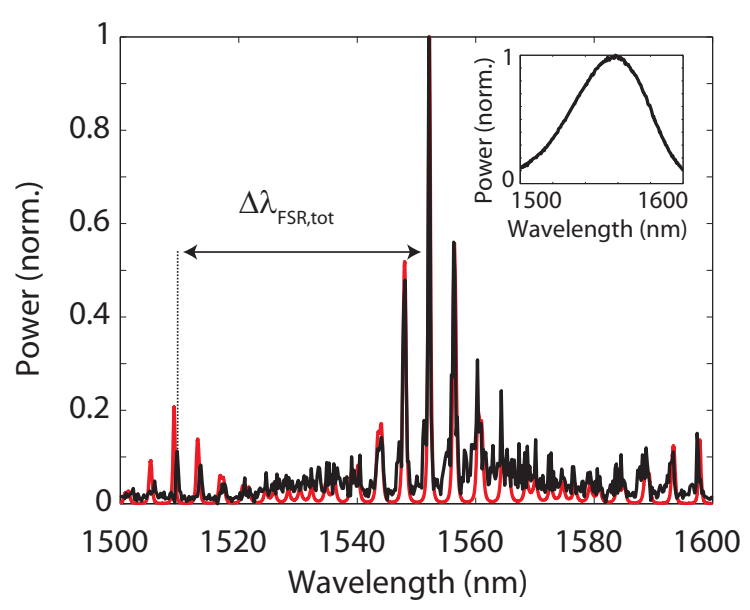

Figure 3.2: Calculated (red line) and measured (black line) reflected power spectra of the MRR mirror, normalized to the maximum power. The output spectrum of the SLD (inset) was used as the input spectrum for the calculation. For the calculations $\kappa^{2}=0.39$, and $n_{g}=1.7293$ was obtained from the fit. The $\delta_{P H R}$ was measured to be 0.56 and the $\Delta \lambda_{F S R, \text { tot }}$ was $42.3 \mathrm{~nm}$.

In Fig. 3.2 it can be seen that the peak power of the reflected light is found at a wavelength of $1552.2 \mathrm{~nm}$. In the figure, the peak reflected power is normalized to unity, but absolute measurements of the power from ports OUT1 and OUT-2 indicate that the internal maximum reflectivity of the MRR circuit (at point S in Fig. 3.1) is about 20\%. The measured peak-height ratio is $0.56 \pm 0.05$. When compensated for the normalized input spectrum of the SLD (inset in Fig. 3.2) this corresponds to a value for the peak height ratio of about 0.6 , which is sufficiently small as compared to the design goal of $\delta_{P H R}<0.63$. To retrieve this and the other characteristic parameters with better precision, the theoretical shape of the reflectivity spectrum, as described by Yariv [25, 34] and Klein [35], and presented in Section 2.3 was fit to the measured spectrum (see red fit curve in Fig. 3.2). To fit the wavelength of the center peak to the measured center peak at $\lambda_{0}=1552.2 \mathrm{~nm}$ the group index, $\mathrm{n}_{g}$, was varied in the calculations. To fit the PHR, the coupling coefficient $\kappa$ was varied. The fit yielded a value of $\mathrm{n}_{g}=1.7293 \pm 0.0001$, which is in good agreement with the expected value $\mathrm{n}_{g}=1.73 \pm 0.1$. The value obtained for the peak height ratio, $\delta_{P H R}=0.55$, corresponds to a value for the coupling coefficient, $\kappa^{2}=0.39 \pm 0.01$, which is in the range of the design goal, $0.3<\kappa^{2}<0.4$. The value for the free spectral range, $\Delta \lambda_{F S R, t o t}$, of the MRR mirror retrieved from the fit is $42.3 \mathrm{~nm}$, which is close to the design value of $\Delta \lambda_{F S R, t o t}=44.0 \mathrm{~nm}$. The residual deviations can be ascribed to a residual fabrication uncertainty for the value of $\kappa$, such as if the coupling coefficients are slightly different for the individual coupling regions that are used for sequential transmission through the two rings. The bandwidth of the center peak, $\Delta \lambda_{F W H M, t o t}$, was retrieved 
to be $0.20 \mathrm{~nm} \pm 0.03 \mathrm{~nm}$ which is even smaller than the design value of $0.43 \mathrm{~nm}$. This deviation can only be explained by the coupling coefficients of the MRRs being rather different due to fabrication errors. In this case, as example, fitting the coupling coefficients to $\kappa_{1}^{2}=0.14$ and $\kappa_{2}^{2}=0.48$ would yield a bandwidth of the center peak of $0.2 \mathrm{~nm}$, while the peak height ratio is equal to that of the measured value. The difference in coupling coefficients from the design value could be explained by a slight lateral displacement of the waveguides during fabrication.

\subsection{Characterization of the free-running diode laser}

The diode laser used in the experiments is a custom anti-reflection coated Cband angled-stripe gain chip (Fraunhofer Heinrich-Hertz-Institut). This laser chip has a specified typical back-facet reflectivity of $85 \% \pm 10 \%$ and an AR coating on the front (output) facet with a maximum reflectivity of $0.1 \%$. The diode laser waveguide forms an angle of $5^{\circ}$ to the surface normal of the output facet. The specified far-field divergence is $19^{\circ} \mathrm{FWHM}$ in both directions, which corresponds to a beam radius at the output facet of $3 \mu \mathrm{m}$ FWHM in both directions. The specified threshold pump current is $17.0 \mathrm{~mA}$.

We verified the threshold pump current to be $\mathrm{I}_{t h}=17.8 \mathrm{~mA}$. The maximum output power was measured to be $7.4 \mathrm{~mW}$ at a pump current of $\mathrm{I}_{L D}=90.0 \mathrm{~mA}$. At approximately twice above threshold $\left(\mathrm{I}_{L D}=30 \mathrm{~mA}\right)$ the observed SMSR was $26 \mathrm{~dB}$ (Fig. 3.3). At higher pump currents $\left(\mathrm{I}_{L D}>60 \mathrm{~mA}\right)$ the SMSR gradually reduced to $20 \mathrm{~dB}$.

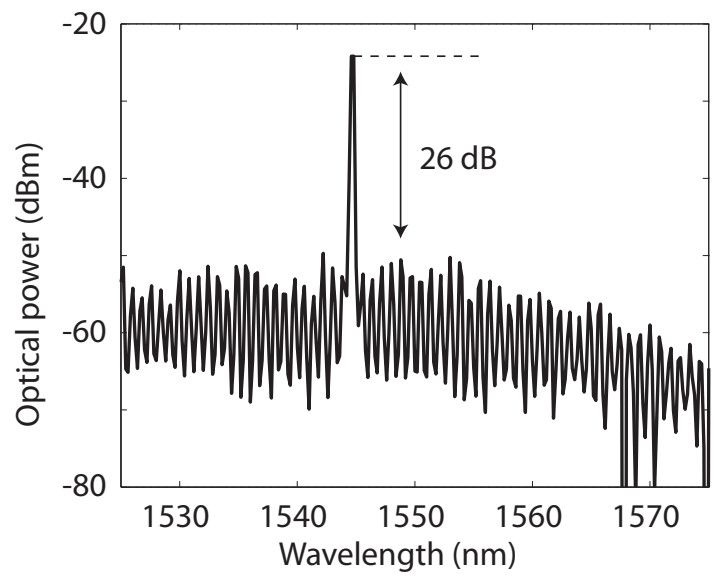

Figure 3.3: Measured output power spectrum of the diode laser on a logarithmic scale, recorded with a pump current of $I_{L D}=30 \mathrm{~mA}$. The measured side-mode suppression ratio was $26 \mathrm{~dB}$. 
The spectral bandwidth was measured to be $0.02 \mathrm{~nm}$ FWHM (i.e., $2.5 \mathrm{GHz}$ ). Because this value is higher than the resolution of the OSA $(0.01 \mathrm{~nm}$, i.e., $1.25 \mathrm{GHz}$ ), we can assume that the spectral bandwidth of the free-running laser is approximately resolved by the OSA.

\subsection{WECSL characterization}

\subsubsection{Optical characterization}

To form the WECSL, the diode was butt-coupled to the MRR mirror. To accurately measure the output power, the out-coupled light was collected (at OUT - 3) using a multi-mode fiber. In our measurements we found that the threshold of the pump current became noticeably lowered, from $17.8 \mathrm{~mA}$ without MRR feedback to a value of $\mathrm{I}_{t h}=5.6 \mathrm{~mA}$. with MRR feedback. The maximum output power was measured to be $1.0 \mathrm{~mW} \pm 5 \cdot 10^{-2} \mathrm{~mW}$, at a pump current of $\mathrm{I}_{L D}=86.7 \mathrm{~mA}$. The observation of a reduced output power compared to that of the free-running diode laser at the same pump current can be largely explained by loss due to a mode mismatch between the gain chip and the waveguide. The loss that we determine using Eq.(2.17) via coupling the sizes of the specified (diode) and calculated (waveguide) mode field is indeed high and amounts to about $88 \%$ loss. Additionally, we expect losses due to Fresnel reflections at the facets of the uncoated waveguide chip (6\% per facet) and the multi-mode fiber used for collecting the light (6\%).

The output spectrum, measured across the range of $1525 \mathrm{~nm}$ to $1575 \mathrm{~nm}$, is displayed in Fig. 3.4. It can be seen that, compared to the free-running case, the maximum value for the SMSR (at a pump current of $\mathrm{I}_{L D}=86.1 \mathrm{~mA}$ ) drastically improved to $50 \mathrm{~dB}$. Depending on the alignment of the gain chip to the MRR mirror's $I N$-port, the SMSR was found to vary between $40 \mathrm{~dB}$ and $50 \mathrm{~dB}$. It should be noted that the maximum displayed power of $-10 \mathrm{~dB}(0.1 \mathrm{~mW})$ is lower than the previously measured, absolute output power of $1.0 \mathrm{~mW}$. This can be addressed to the mode mismatch of the mode field diameter (MFD) of the waveguide output with that of the MFD of the single-mode fiber, used for recording. 


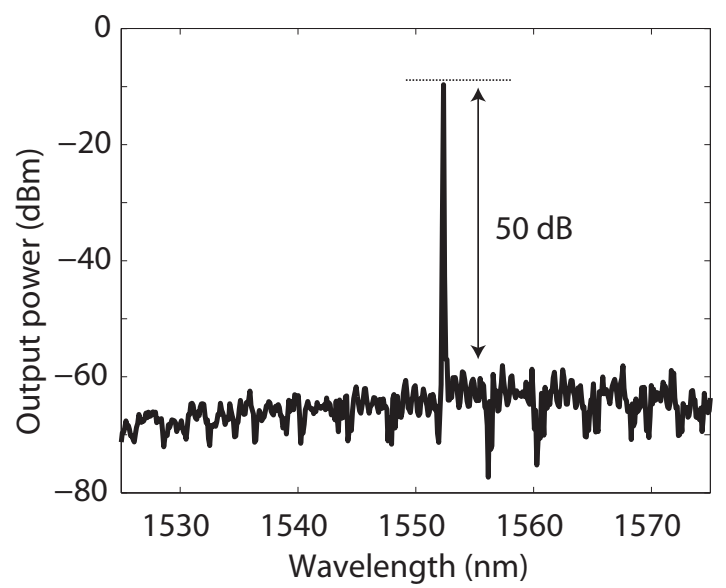

Figure 3.4: Measured output power spectrum of the WECSL on a logarithmic scale. The measured side-mode suppression ratio (SMSR) was $50 \mathrm{~dB}$. With the relative measure of power expressed by the logarithmic $\mathrm{dBm}$ scale, $1 \mathrm{~mW}$ corresponds to $0 \mathrm{dBm}$. Similarly, the measured SMSR of $50 \mathrm{~dB}$ corresponds to a ratio of $10^{5}: 1$.

To measure the tuning range of the WECSL, we applied a voltage only to the heater of the MRR with radius $R_{1}=50 \mu \mathrm{m}$. We expect to see wavelength switches of the size of the FSR of $R_{2}$ (i.e., $4.0 \mathrm{~nm}$ ). Fig. 3.5 summarizes the measured result, showing several superimposed spectra as recorded sequentially with seven different heater voltages. 


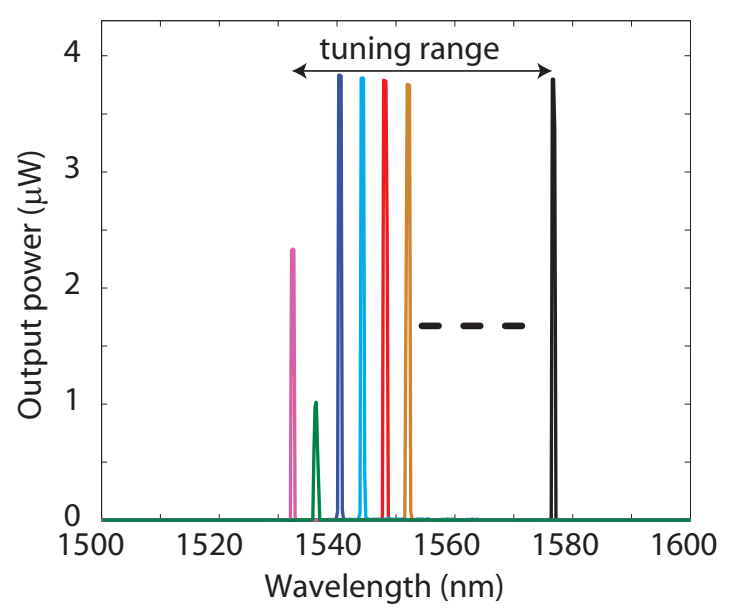

Figure 3.5: Superposition of several output spectra of the WECSL, obtained by stepwise increasing the heater voltage of a single $M R R$ (with radius $R_{1}$ ). The overall spectral coverage, indicated as tuning range, was 42.3 $\mathrm{nm}$. The different colors indicate individual measurements.

It can be seen that the generated wavelengths comprise a range of $42.3 \mathrm{~nm}$. This means the tuning range of the realized WECSL covers even more than the entire $35 \mathrm{~nm}$ wide telecommunication C-band.

To quantify the minimum time in which the laser wavelength can be set to a different value we define the switching time between two preset wavelengths as the time interval in which the light power received at these wavelengths changes from $10 \%$ to $90 \%$. We use a computer controlled voltage supply, connected to a home-built power amplifier to drive the heaters. The output ports from the power amplifier were connected to the electrical contacts on the waveguide chip. By applying a voltage to only the heater of the ring with radius $R_{1}=50 \mu \mathrm{m}$, we expect to see wavelength switches of the size of the FSR of the ring with radius $R_{2}$ (i.e., $4.0 \mathrm{~nm}$ ). A stable and reproducible wavelength switch was observed from $\lambda=1552.2 \mathrm{~nm}$ to $\lambda=1548.2 \mathrm{~nm}$ for a voltage step from $0 \mathrm{~V}$ to $2.3 \mathrm{~V}$. To observe power changes at these two wavelengths with sufficiently high speed, beyond the recording speed provided by the OSA, the output is directed onto a reflective diffraction grating $(600$ lines $/ \mathrm{mm})$ at normal incidence. A photodiode is placed in one of the two transverse positions, $2 \mathrm{~m}$ from the grating, corresponding to diffracted light at a wavelength of either $\lambda=1552.2 \mathrm{~nm}$ or $\lambda=1548.2 \mathrm{~nm}$. Fig. 3.6 shows the photodiode signal as a function of time. The traces show a step-like shape, with a switching time of $200 \mu \mathrm{s} \pm 40 \mu \mathrm{s}$. 
(a)

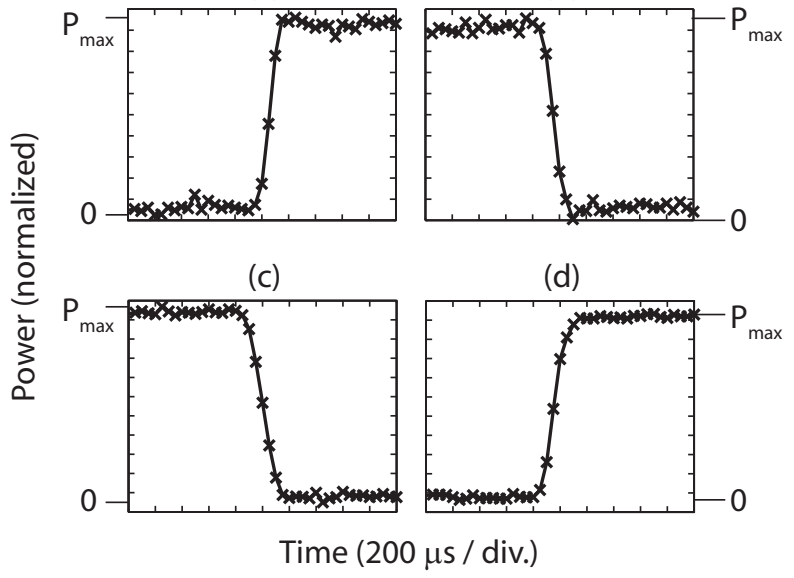

Figure 3.6: Measured power from which laser switching times are derived. In (a) and (b) the photodiode is aligned to detect light with a wavelength of 1552.2 $\mathrm{nm}$, and in (c) and (d) the photodiode is aligned to detect light with a wavelength of $1548.2 \mathrm{~nm}$. In $(a)$ and $(c)$ the laser is switched from $1548.2 \mathrm{~nm}$ to $1552.2 \mathrm{~nm}$, whereas in (b) and (d) the laser is switched from 1552.2 $\mathrm{nm}$ to $1548.2 \mathrm{~nm}$. Per graph, the measured power was normalized to the maximum measured power, $P_{\max }$.

To measure the spectral bandwidth of the optical output of the WECSL with high resolution (higher than provided by the OSA), we used a delayed selfheterodyne interferometer as described in $[46,47]$ with a delay line of $16 \mathrm{~km}$ of single-mode fiber. The schematic of this measurement setup is shown in Fig. 3.7 For this measurement we used a $20 \mathrm{GHz}$ optical detector (Discovery Semi DSC30s), an acousto-optic modulator (AOM) (AA Opto Electronic, MT80-IIR60-F10-PM0.5-130.s), an AOM driver (ISOMET 232A-2), an Erbium doped fiber amplifier (EDFA)(Firmstein Technologies Inc. PR25R) and an RF spectrum analyzer (Agilent Signal Analyzer MXA N9020A).

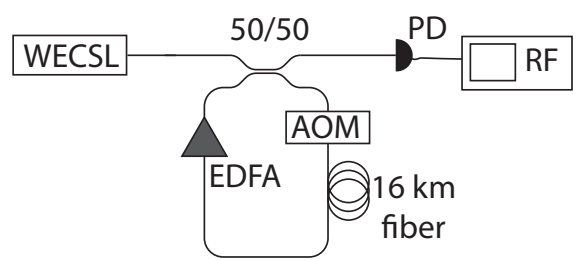

Figure 3.7: Schematic setup of the delayed self-heterodyne interferometer, using a $16 \mathrm{~km}$ long fiber in one of the arms, to measure the spectral bandwidth of the WECSL. The setup comprises an acoustooptic modulator (AOM), an erbium doped fiber amplifier (EDFA), a photodiode $(P D)$ and a radio frequency spectrum analyzer $(R F)$. 
The measured power spectrum of the RF-beat signal is shown in Fig. 3.8, where the black dots indicate the measurement data and the red line shows a Lorentzian fit. As can be seen by the excellent matching of the fit curve to the experimental data, the measured beat spectrum is to high precision of Lorentzian shape. The $3 \mathrm{~dB}$ bandwidth of the fit is $50 \mathrm{kHz}$, which corresponds to a $25 \mathrm{kHz}$ FWHM laser bandwidth. The phase noise of the RF oscillator is in the $\mathrm{Hz}$ regime, such that it does not significantly contribute to the measured bandwidth of the laser.

This laser bandwidth of $25 \mathrm{kHz}$ is surprisingly narrow, when only the physical length of the external waveguide cavity (a few $\mathrm{mm}$ ) is taken into account. As comparison, to achieve a similar bandwidth with a diode laser with an external cavity using a grating, the physical length of the external cavity would have to be several $\mathrm{cm}$.

The narrow bandwidth can be explained by the following two physical effects. First, because the resonant light performs multiple roundtrips whereby the MRRs introduce an extra optical delay [48]. This results in an increase of the cavity photon lifetime, due to the relatively high quality factors (Q-factors) of the MRRs.

Second, unlike the diode chip back facet that provides a spectrally broadband reflection, the MRR mirror provides a frequency selective feedback. This narrows the laser bandwidth even further, which can be viewed as due to chirp reduction [49] and optical self-locking [32]. Using Eq.(2.16) one can calculate the minimum expected bandwidth of the laser. For the calculation we used the following set of parameters which are known from the diode chip specifications and from the above reported characterization of the waveguide MRR mirror. Specifically, the values used are as follows. The diode laser material has an index of $n_{d}=3.16$ and the physical length of the chip is $L_{d}=400 \mu \mathrm{m}$. The MRR mirror has a group index of $n_{e c}=1.73$ and an effective path length, $L_{e c}=4.1 \mathrm{~mm}$ (measured physical length), which includes the multiple roundtrips in the MRRs $\left(\left(2 \pi \cdot\left(R_{1}+R_{2}\right)\right) / \kappa^{2}\right)$ [48]. The Finesse of the MRR mirror is calculated as $F=\Delta \nu_{F S R, t o t} / \Delta \nu_{F W H M, t o t}=$ $\Delta \lambda_{F S R, t o t} / \Delta \lambda_{F W H M, t o t}[31]$, which yields a value for the finesse of the external cavity of $F_{e c}=211.5$, using the measured values for the MRR mirror bandwidth, $\Delta \lambda_{F W H M, t o t}$, of $0.2 \mathrm{~nm}$ and the free spectral range $\Delta \lambda_{F S R, t o t}=$ $42.3 \mathrm{~nm}$. For the diode laser cavity the free spectral range is calculated to be $\Delta \nu_{F S R, d}=120 \mathrm{GHz}$ (Eq.(2.5)) and the spectral bandwidth of the diode cavity modes is $\Delta \nu_{F W H M, t o t}=343 \mathrm{GHz}$ (Eq.(2.10), which is calculated by using the reflectivities for the back and front facet respectively, $R_{b f}=0.85$ and $R_{f f}=1.46 \cdot 10^{-8}$ ), as described in Section 2.3. The calculations yield a finesse of the diode laser cavity of $F_{d}=0.35$. Using Eq.(2.16), where the effective increase in cavity length and the increase in finesse both reduce quadratically the Schawlow-Townes limit, and where we have assumed to well approach the situation of low feedback losses with $\beta=1$, we calculate the ultimately lowest theoretical bandwidth for this WECSL to be as low as $7.5 \mathrm{~Hz}$. 
These calculations show that there are two major improvements in designing a new MRR mirror, that could reduce the measured bandwidth. First, the cavity photon lifetime can be increased through decreasing the coupling coefficient for the MRRs in the feedback path. Second, by decreasing the cavity-internal loss by mode matching the waveguide to the gain chip, the cavity photon lifetime can be increased.

In the experiments reported here, this theoretical limit is not achieved. We believe this is mainly due to the mode mismatch in coupling between the gain chip and the waveguide, which leads to increased intra cavity losses (i.e., which lowers the value of $\beta$ ). For the experimental setup described above, this first part amounts to a loss of $88 \%$. Adding losses due to reflections at the facet of the waveguide $(6 \%)$ and the internal maximum reflectivity of the MRR mirror (20\%), we come to a value of $\beta=2.5 \cdot 10^{-3}$. Inserting this into Eq.(2.16), predicts an expected bandwidth of $3.1 \mathrm{kHz}$. This value is at least roughly comparable to the measured bandwidth of $25 \mathrm{kHz}$. Remaining deviations can be addressed to waveguide losses, and alignment errors of the diode laser with regard to the waveguide. The main source of waveguide losses are caused by the waveguide coupler that diverts light to the measurement channel (C in Fig.3.1) which is passed twice per roundtrip and contributes to an estimated value of $10 \%$ per roundtrip. The corresponding increase in linewidth would be about $11 \%$.

Regarding losses by misalignment, we have estimated for our mode overlap conditions that a transverse misalignment of about $100 \mathrm{~nm}$ would already yield an increase of the laser bandwidth by several kHz (Eq.(2.17)). Also, we believe that acoustic vibrations of the residual gap between gain chip and waveguide chip cause the cavity loss and length to fluctuate, both of which can lead to spectral broadening (via amplitude modulation in the case of cavity loss and increased phase noise in the case of length modulation). However, if these effects had dominated the laser bandwidth, they should have shown up in a deviation from the measured Lorentzian line shape in Fig. 3.8 [29].

Nevertheless, a theoretical minimum laser bandwidth of $7.5 \mathrm{~Hz}$ shows a significant potential of such WECSLs as future light sources with high spectral purity. This potential is, in particular, important because it shows that ultralow spectral bandwidths can be realized in a chip-sized format, suitable for integration with subsequent waveguide circuitry. 


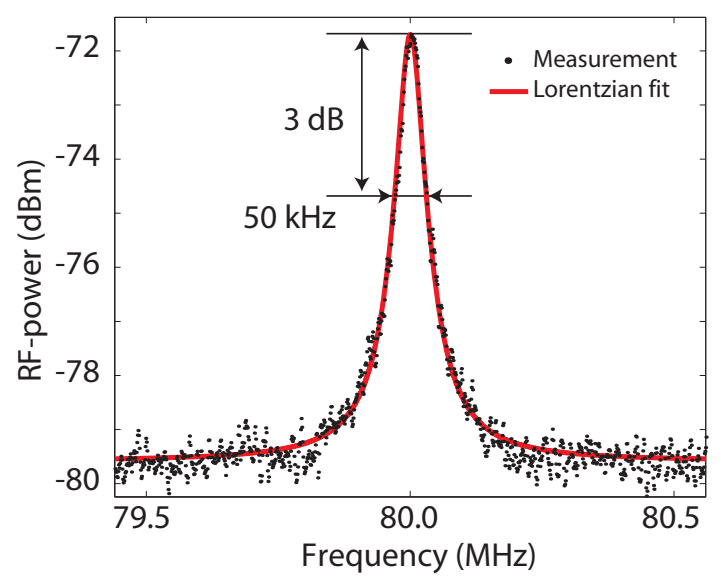

Figure 3.8: Self-heterodyne beat spectrum the WECSL. The black dots show the measured RF beat spectrum and the red line shows a Lorentzian fit with a $3 \mathrm{~dB}$ bandwidth of $50 \mathrm{kHz}$. The measurement reveals a laser bandwidth of $25 \mathrm{kHz}$.

To summarize, our measurements show that, compared to the free-running laser diode, the external cavity strongly improves the SMSR (by $30 \mathrm{~dB}$, from $20 \mathrm{~dB}$ to $50 \mathrm{~dB}$ ). The measured tuning range of $42.3 \mathrm{~nm}$ covers the entire telecom C-band and the switching time between two wavelengths is $200 \mu \mathrm{s}$. The laser bandwidth is as narrow as $25 \mathrm{kHz}$ and bears the potential of further significant reduction, possibly in the range below $10 \mathrm{~Hz}$.

\subsubsection{Injection locking}

In our experiments we have used a setup which is very similar to that described by Stover and Steier [13], as shown in Fig. 3.9. Light from the slave laser does not influence the master laser, due to the optical isolator. The output light of both master and slave laser are collected on a photodiode. The beat signal of the frequencies of the master and slave laser is displayed on an RF spectrum analyzer. The master laser's frequency is to be swept over the expected injection locking range of the slave laser, as described in Section 2.4.1, while observing the beat note of the two lasers. If injection locking occurs, this should show up as the beat note vanishing. However, we expect that the laser wavelength of the WECSL master laser cannot be swept with the required precision, presumably in the tens of $\mathrm{MHz}$ range. The reason is that the wavelength tuning mechanism, heating of MRRs to modify their refractive index, is relatively coarse. To circumvent this problem we provide a precisely tunable light frequency with optical side band generation. This is achieved with a Mach-Zehnder interferometer based optical modulator, which induces sidebands at a frequency $\Omega$ to the master laser frequency, $\nu_{m} \pm n \cdot \Omega$, with $n$ an integer. The RF oscillator for the available modulator (Avanex PowerLog FA 20) can be tuned with a 
sub-kHz precision over a wide range of radio to microwave frequencies (a few $\mathrm{kHz}$ to $20 \mathrm{GHz}$ ) which exceeds the expected locking range. The efficiency of the modulator is such that the power in the first sidebands $\left(\nu_{m} \pm \Omega\right)$ each contain $15 \%$ of the incident power.

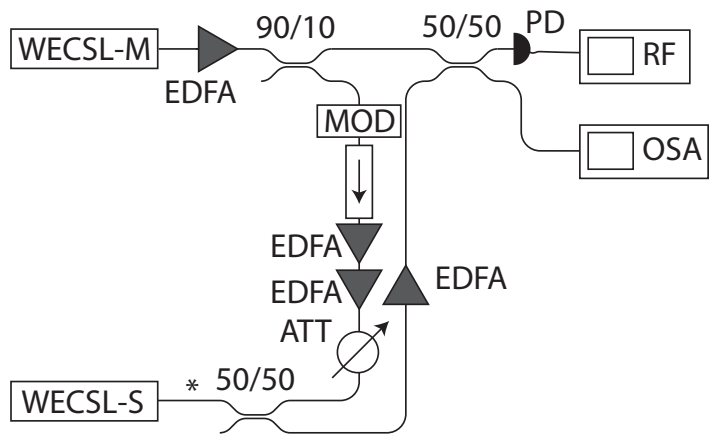

Figure 3.9: Schematic of the injection locking setup. The master laser is marked as WECSL-M, and the slave as WECSL-S. Light from the master laser is sent through the optical modulator (MOD), sent through an optical isolator $(\rightarrow)$ and amplified in two steps by an erbium doped amplifier (EDFA). Thereafter, the light passes an optical attenuator (ATT) and a coupler (50/50), before it is injected into the slave laser. Superposition of light from the master and slave is done using a 90/10 and 50/50 coupler (master) and a 50/50 coupler (slave). The beat signal is recorded with a photodiode (PD) and an RF spectrum analyzer (RF), under monitoring with an optical spectrum analyzer (OSA).

The detailed description of the experimental setup is as follows. The fibercoupled output light of the master laser is amplified in an Erbium doped fiber amplifier (EDFA). A total of four available EDFAs are used from two different manufacturers: Firmstein Technologies Inc. (type PR25R) and Alcatel (type 1686WM). After the first EDFA, a 90/10 coupler splits the light into two paths. The $90 \%$ path goes towards a fast photodiode (Discovery Semi DSC 30S, with an $\mathrm{RF}$ bandwidth of $20 \mathrm{GHz}$ ). The $10 \%$ path enters the optical modulator, which is driven by a vector signal generator (Agilent Technologies, E8267D). The modulated light goes through an optical isolator, through two EDFAs and an optical attenuator to a 50/50 coupler, which diverts $50 \%$ of the light towards the slave laser. The output light from the slave laser is coupled through the same 50/50 coupler towards a fourth EDFA. Behind the amplifier, the light is superimposed with light from the master laser, via a 50/50 coupler and collected on the photodiode as well. The RF part of the photodiode signal, which is the beat note of the master and slave laser light fields, is recorded by an RF signal analyzer (Agilent Signal Analyzer MXA N9020A, with an RF bandwidth of $10 \mathrm{~Hz}$ to $26.5 \mathrm{GHz}$ ). To measure whether the beat frequency is 
within the $20 \mathrm{GHz}$ tuning range of the signal generator, the other arm of the latter 50/50 coupler is connected to an optical spectrum analyzer (OSA) (Ando AQ6317).

These experiments were carried out as follows. First, as a calibration we measure the power through the 50/50 coupler from the master laser (after amplification) at the position marked by an asterisk (*) in Fig. 2.7. At the same position, we measure the output power of the slave laser. Subsequently correcting for the modal mismatch between the waveguide of the WECSL and the fiber, allows us to determine the input and output power of the slave laser $\left(P_{\text {in }}\right.$ and $P_{\text {out }}$, respectively). We then measure the difference frequency between the master and slave laser, $\left|\nu_{m}-\nu_{s}\right|$. If required, we tune the frequency difference to be within $20 \mathrm{GHz}$ of each other. In our experiment, the master and slave laser were tuned to a frequency difference of $\left|\nu_{m}-\nu_{s}\right|=11.2 \mathrm{GHz}$, around a center frequency $\nu_{0}=194.05 \mathrm{THz}$. Hereafter, we set the optical modulator to modulate the light of the master laser in the range of this difference frequency. If we observe two beat notes, which occur as two separate peaks on the RF spectrum analyzer, one of these peaks is the beat note between master and slave laser, the other is the beat note between the master laser's center frequency and its sidebands $(\Omega)$. In such case we conclude that the master and slave laser are not injection locked yet. By sweeping the optical modulation frequency over a range of about one to two $\mathrm{GHz}$ around the named frequency difference of $\mid \nu_{m^{-}}$ $\nu_{s} \mid$, we record between which frequencies one of the beat notes becomes equal to the beat note of the other. This is then recorded as the locking range. After measuring the locking range, we repeat the sweeping experiment for different attenuations (in steps of $0.5 \mathrm{~dB}$ ) of the master laser's power.

The measured output of the slave laser (without injection locking), coupled into the fiber between the slave laser and the 50/50 coupler (position marked by the asterisk in Fig. 2.7), is measured to be $2.09 \cdot 10^{-2} \mathrm{~mW}$. The power through the 50/50 coupler from the master laser (after amplification) is measured to be $2.6 \mathrm{~mW}$. Recalling the $15 \%$ power fraction in the side bands means that at the sideband frequencies $\nu_{m} \pm \Omega$, the optical power is $0.39 \mathrm{~mW}$. When applying the power calibration described above, we obtain for the injected power a value of $P_{\text {in }}=1.46 \cdot 10^{-2} \mathrm{~mW}$ and for the output power of the slave laser a value of $P_{\text {out }}=0.58 \mathrm{~mW}$. The given value for the injected power was determined with the attenuator set to zero $\mathrm{dB}$ (no attenuation). 


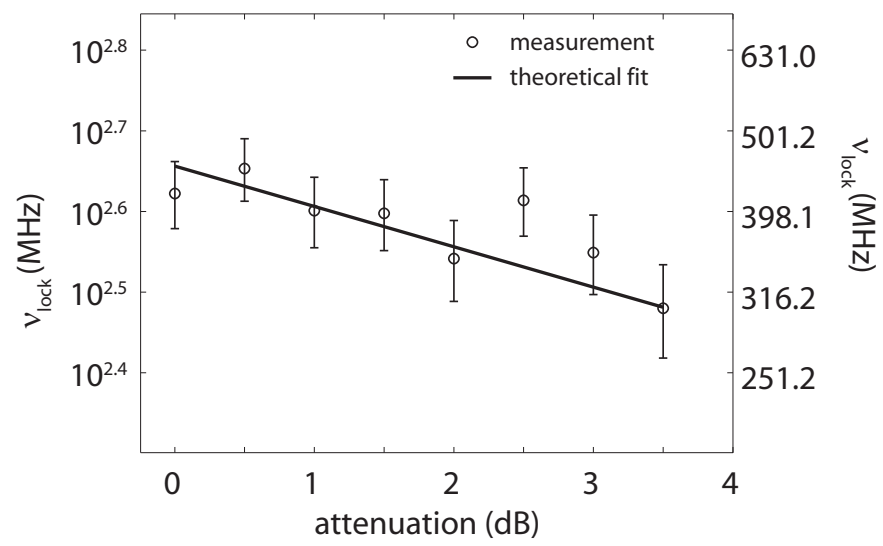

Figure 3.10: Measured injection locking range of a WECSL vs. the injected power from another WECSL (circles). The injected power is expressed as the attenuation factor used for the master laser injection. ( $0 \mathrm{~dB}$ corresponds to $P_{\text {in }}=1.46 \cdot 10^{-2} \mathrm{~mW}$ ). The output power of the slave laser is $0.58 \mathrm{~mW}$. The straight line is a best fit of Eq.(2.18) to the data, representing a square-root function. Note that both vertical axes and the horizontal axis are logarithmic.

The results of the injection locking experiment are shown in Fig. 3.10, with on the horizontal axis the attenuation of the injected master laser radiation (in $\mathrm{dB}$ ) and on the vertical axis the locking range, $\Delta \nu_{l o c k}$, on a logarithmic scale. The error bar indicates the readout error of $\pm 50 \mathrm{MHz}$ from the RF spectrum analyzer. It can be seen that the injection locking range varies between approximately 300 to $400 \mathrm{MHz}$ within the shown attenuation of the injection. It should be noted that for attenuations $\geqslant 4.0 \mathrm{~dB}$, no more injection locking was observed.

The solid line in Fig. 3.10 shows a fit of the theoretically expected variation of the locking range, expressed by Eq.(2.18), to the measurements. The prediction of Eq.(2.18), due to the square-root dependence of the locking range vs. injected power, follows a straight line with a slope of $-1 / 2$ in Fig. 3.10, due to the double-logarithmic way of plotting. In this fit, the Q-factor of the slave laser was varied to achieve the best agreement with the experimental data, yielding a value of $\mathrm{Q}=7.7 \cdot 10^{4}$.

For comparison with earlier results, from this Q-factor one can estimate the bandwidth of the slave laser, using the original expression of the SchawlowTownes limit for diode lasers with a Fabry Perot cavity, as described in Eq.(2.15). For this purpose we calculate the cavity bandwidth, assuming that injection locking of a WECSL can be described similarly to that of a common Fabry Perot laser. By using the definition of the Q-factor, $Q=\nu_{\text {slave }} / \Delta \nu_{c}$, and using an estimated linewidth enhancement factor, $\alpha=5$, we calculate a Schawlow-Townes limit of $\Delta \nu_{S T}=114 \mathrm{kHz}$. This value is comparable to the earlier measured $25 \mathrm{kHz}$, if assuming that in the injection locking experiments 
there was some additional cavity loss caused by a non-optimum alignment as was discussed in Section 3.4.1.

The measurements show, that WECSLs are a suitable candidate to be used in manifold in an optical beam forming network of a phased antenna array, as proposed in Section 2.4.

\subsection{Conclusions}

In this chapter we have presented a basic characterization of a waveguide based external cavity semiconductor laser (WECSL). The investigated example of a WECSL consists of a diode laser with feedback from an external cavity formed by a frequency selective waveguide circuit called a micro ring resonator (MRR) mirror. The MRR mirror comprises two micro ring resonators with radii $R_{1}=$ $50 \mu \mathrm{m}$ and $R_{2}=55 \mu \mathrm{m}$. The measured free spectral range (FSR) of the reflectivity spectrum provided by the double ring feedback is $\Delta \lambda_{F S R, t o t}=$ $42.3 \mathrm{~nm}$, with a peak height ratio of $\delta_{P H R}=0.55$, and with a couping coefficient for the MRRs of $\kappa^{2}=0.39$. The bandwidth of the reflected center peak was $0.2 \mathrm{~nm}$. These values agree well with calculated and designed values for the MRR mirror, as discussed in Chapter 2.

The WECSL was formed by connecting the MRR mirror to a diode laser gain chip. Measurements showed that the WECSL is tunable over the full free spectral range of $42.3 \mathrm{~nm}$. Rapid switching between two arbitrary wavelengths was demonstrated with a switching time as short as about $200 \mu \mathrm{s}$. The WECSL offers a small spectral bandwidth of $25 \mathrm{kHz}$, with a SMSR of $50 \mathrm{~dB}$. The extrapolated fundamental lower bandwidth (Schawlow-Townes) limit, reduced by the MRR-based enhancement of the cavity photon lifetime and reduced by the spectral narrowing of the external feedback, is in the order of only $7.5 \mathrm{~Hz}$, provided that the feedback losses can be minimized to reach values for $\beta$ that approach unity. This indicates that, via technical improvements (such as higher optical coupling between diode laser and waveguide) it should be possible to reduce the experimental bandwidth of the laser by several orders of magnitude.

A measurement of the effective Q-factor of the complex resonator structure of a WECSL after assembly was done via injection locking. In these experiments the Q-factor was determined to be $7.7 \cdot 10^{4}$. For a Fabry Perot diode laser this would correspond to a Schawlow-Townes limit of $114 \mathrm{kHz}$, which is comparable to the earlier measured $25 \mathrm{kHz}$, taking additional misalignments into account.

The narrow bandwidth and great potential for significant further narrowing, fast wavelength switching, wide wavelength tunability and and suitability for injection locking make this WECSL a candidate for advanced applications such as in broadband phased array antenna systems. 


\section{4 SEGA mode locking theory}

In this chapter we present a novel laser mode locking scheme which we call "separate gain mode locking" (SEGA mode locking) and discuss its unusual properties. Using a numerical model, the basic feasibility of this method is investigated. We start with possible applications of mode locked lasers with a high repetition rate and high average output power in Section 4.1. In Section 4.2 the problems for standard mode locked lasers in achieving a combination of high repetition rate and high average output power are described. As a possible solution, we introduce the concept and, for definiteness, also an envisioned experimental setup of SEGA mode locking in Section 4.3. The theoretical background and the model developed to quantitatively describe the SEGA mode locking approach are discussed in Section 4.4. The choices for the critical parts of the model are described in more detail in the respective Subsections 4.4.2, 4.4.3 and 4.4.4. The results of the modeling provide a set of design parameters within which mode locking could occur, as described in Section 4.5. These design parameters form the basis for the experiments discussed in Chapter 5.

\subsection{Introduction}

Ultra-short laser pulses are widely used in scientific research ranging from fundamental physics to biology, and find use in many applications such as for material processing or in telecommunications $[50,51]$. The most common method to generate ultra-short pulses is passive mode locking because this can yield particularly short pulse durations in the femtosecond range. However, as will be discussed, certain combinations of repetition rates, output powers, and pulse durations are difficult to achieve. For instance, higher average powers generally 
require larger cavities, while a high pulse repetition rate requires a short cavity length. This is because the repetition rate scales inversely with the length of the laser cavity [52].

The named connection between repetition rate and cavity length implies that high repetition rate and high average power are incompatible using current approaches. To illustrate this, let us consider a very short cavity length of only a few hundred $\mu \mathrm{m}$, such as can be realized in semiconductor lasers. Indeed, very high repetition rates of hundreds of $\mathrm{GHz}$ are obtained with such lasers [14]. However, the short cavity length limits the roundtrip gain and thus also the average power, typically to below a milliwatt range with such semiconductor lasers. Extending the cavity length allows for higher average output power, but also lowers the repetition rate and thereby introduces other limitations. A first restriction, noticeably in semiconductor lasers, comes from the limited lifetime (nanoseconds) of the upper state, such as is given by spontaneous emission. When lowering the repetition rate to below a few $\mathrm{GHz}$, amplified spontaneous emission occurs between pulses [53], which depletes the inversion and reduces the temporal coherence of the output. A second restriction is distortion of the pulse shape by dispersion, or by gain saturation and other nonlinear effects. For instance, Ti:Sapphire oscillators have their average output power limited to a few Watts by such effects.

The described incompatibility limits applications where nonlinear processes need to be driven at the highest repetition frequency. For example, photomagnetic switching (as described by Hansteen et al. [8]) requires an intensity of $50 \mathrm{GW} / \mathrm{cm}^{2}$ over a pulse duration of 100 fs to initiate a single switching operation. From this pulse duration, the maximum switching rate would be in the order of several THz. This is indeed an impressive speed for the envisioned optical writing of bits on a magnetic disc. However, a $1 \mathrm{THz}$ pulse repetition rate laser, focused to a diffraction limited spot, therefore, requires an average power of approximately $10 \mathrm{~W}$, which is well beyond the range of the capabilities of current mode locked diode lasers [54].

In the remainder of this chapter a novel mode locking method is described, that is capable of overcoming the named limitations by producing a high repetition rate as well as a high average output power, by decoupling the repetition rate from the length of the cavity [15]. In this scheme, a large set of singlefrequency continuous-wave lasers oscillate by amplification in spatially separated gain media. They are mutually phase-locked by nonlinear feedback from a common saturable absorber. As a result, ultra-short pulses are generated. This method is what we call separate gain (SEGA) mode locking. Compared to standard mode locked lasers, the new scheme offers three significant benefits. The light that is amplified in each separate gain element is spectrally narrowband and continuous wave, thereby avoiding issues related to group-velocity dispersion and nonlinear effects that can perturb the pulse shape. The spectral separation of the set of frequencies on which the laser oscillates, and therefore the pulse repetition rate, is controlled by the dispersion of resonator-internal optical elements and the resonator geometry, not by the cavity length. Finally, 
the bandwidth of the laser can be dynamically controlled by switching sets of gain elements on and off during laser operation. This scheme offers a new route to mode locked lasers with high average output power, repetition rates that can be scaled into the $\mathrm{THz}$ range, and a bandwidth and pulse duration that can be dynamically controlled. This approach is particularly suited for implementation using semiconductor diode laser bars. The reason is that diode lasers can operate with high roundtrip losses, due to high amplification factors provided by semiconductor gain materials. An important advantage is also that diode laser arrays are available with hundreds of emitters in a format that is not much bigger than diode lasers with single emitters, with significant output powers in the range of tens to hundreds of Watts.

\subsection{Standard mode locking}

Mode locking is described comprehensively in various textbooks [55, 56]. In general the intracavity losses are varied by some optical power modulator, called the mode locker. In this Thesis we will consider passive mode locking via a semiconductor saturable absorber mirror (SESAM [57]) as mode locker, because this method seems to offer the best compromise between an ultrafast response and a low peak power required for saturation.

In a simplified description in the time domain a SESAM exhibits a constant optical absorbance in time for incident light at a low intensity. This absorbance decreases with increasing light intensity. When employed as cavity mirror the SESAM provides higher roundtrip losses for continuous wave (CW) light (i.e., low intensity) compared to the losses for pulsed light (i.e., high peak intensity). This leads to pulsed operation because the temporal modulation of the light caused by the SESAM is synchronized to the roundtrip time of the optical pulse. A more detailed description on the working principles of SESAMs can be found in, e.g., Keller et al. [14, 57] and Grange et al. [58], which is partially summarized in Section 4.4.3.

An alternative description of mode locking can be given in the frequency domain. In this case, the laser is seen as oscillating on many longitudinal resonator modes, with equally spaced frequencies $\left(\nu_{n}\right)$, i.e., $\nu_{n+1}-\nu_{n}=\Delta \nu$, where $\Delta \nu$ is the difference between the $n^{t h}$ and the $n+1^{\text {st }}$ frequency (with integer $n$ ). Pulses occur when the individual light frequencies are given a certain fixed (temporally constant) phase relation. Pulses commonly known as Fourier limited pulses are formed when the phase difference $\Delta \phi$ of adjacent frequencies independent of the mode number n, i.e., $\Delta \phi=\phi_{n+1}-\phi_{n}$ is the same for all $n$, with $\phi_{n}$ the phase of the $n^{\text {th }}$ mode. This relation is also called a linear phase relation or a linear phase spectrum. These phases can be locked into such phase relation by means of the mode locker via the following mechanism. The mode locker modulates the individual frequencies with a period $\Omega$, thus producing side bands at $\nu_{n} \pm \Omega$, the phases of which are fixed with regard to that of the carrier $\nu_{n}$. When the side bands coincide with the neighboring 
frequency $(\Omega \approx \Delta \nu)$ within the locking range of the laser (for a definition see Chapter 2), the side band phases determine the phases of the neighboring modes through injection locking. Fourier transforming the resulting amplitude and phase spectrum into the time domain gives a periodic function, i.e., a periodic pulse train.

When a higher repetition frequency of pulses $\left(f_{\text {rep }}\right)$ is required from a standard mode locked laser, the relation between repetition frequency, $f_{\text {rep }}$, frequency spacing, $\Delta \nu$ and roundtrip time, $t_{p}$, has to be investigated. In the frequency domain, the frequency spacing of the spectrum is equal to the repetition rate, described as

$$
\Delta \nu=\frac{1}{t_{p}}=f_{\text {rep }}
$$

Eq.(4.1) indicates that a higher repetition rate can be achieved by reducing the roundtrip time. $\Delta \nu$ is also called the free spectral range (FSR, $\Delta \nu_{F S R}$ ) of the cavity, i.e., $\Delta \nu=\Delta \nu_{F S R}$. In the case of a standing wave cavity, the relation between the FSR and the optical length of a resonator $\left(L_{e f f}\right)$ is given by

$$
\Delta \nu_{F S R}=\frac{c}{2 L_{e f f}},
$$

with $c$ the speed of light in vacuum.

These expressions seem to imply that, if the goal is to increase the repetition rate of a mode locked laser, this requires to reduce the cavity length accordingly. However, decreasing the cavity length has physical limits. Specifically, the cavity length cannot be made shorter than the gain material. A further shortening of the cavity (and thus also the gain material) would reduce the average output power of the laser, which is not desired. Another method of increasing the repetition rate, but without decreasing the cavity length is called harmonic mode locking [59]. The method is based on placing an etalon inside the laser cavity. The frequency spacing of the light fields that can oscillate inside the laser cavity is restricted to the longitudinal resonator modes of the etalon. In the time domain this can be seen as the generation of additional (echo) pulses, caused by the two reflecting facets of the etalon. This way multiple pulses run through the cavity simultaneously and the length of the etalon can be changed independent of the length of the gain material as long as the FSR of the etalon is a multiple integer of the FSR of the laser cavity. A recent (2012) publication [60] using this method presented a vertical external cavity surface emitting laser (VECSEL) harmonically mode locked to produce a repetition rate of $175 \mathrm{GHz}$, with pulse durations of $400 \mathrm{fs}$ and an average output power of $300 \mathrm{~mW}$.

What these mode locking methods have in common is the coupling of repetition rate and the cavity length (Eq.(4.2)). When extremely high repetition rates, combined with high peak power are required, conventional mode locking 
methods are insufficient.

However, when looking at the Fourier transform of a periodic train of pulses, there is no such requirement for the named coupling. All that is required to generate a pulse train is to generate a line spectrum with equidistant frequencies and an associated linear phase spectrum. It is this notion that forms the basis for the SEGA mode locking concept. Instead of using a laser with a single cavity that generates a set of equally spaced light frequencies, we consider to use an entire set of high-power CW lasers. Each of these lasers can oscillate as based on its own cavity and with each laser frequency tuned to obtain evenly spaced output frequencies. In this setting, a nonlinear optical coupling between the lasers is introduced in order to control their relative phases, to generate a pulse train.

\subsection{Concept}

In our SEGA mode locked laser multiple single-frequency lasers are phaselocked via a common non-linear optical element. Our approach may be applied to all types of lasers, however, for clarity and relevance, we concentrate on a specific type of diode lasers. To illustrate the scheme and investigate its feasibility with current technology, a specific setup is discussed. It has an external cavity diode laser using bulk optical elements as shown in Fig. 4.1, although one could also think of other designs, e.g., using an array of waveguide circuits to control an array of diode lasers. In Chapter 5, a first experimental step into this direction is presented.

In the SEGA mode locked laser, as schematically depicted in Fig. 4.1, the gain is provided by a multiple-emitter diode bar in which the individual gain elements are sufficiently separated from each other to exclude mutual evanescent field coupling. The bar is equipped with a high-reflection (HR) coating on the back facet and an anti-reflection (AR) coated front facet. The AR coating avoids undesired feedback from the front facet as was described in Chapter 2.2.1 to ensure that the single emitters amplify light without imposing an additional longitudinal-mode structure. The cavity contains focusing optics, a diffraction grating and a SESAM. The external cavity confines the feedback to each emitter to a narrow frequency range, with an approximately uniform spacing between the frequencies for neighboring elements. This is achieved via the diffraction grating such that the -1st order diffraction angle is common to all emitters, as described in Refs. [61, 62]. This common arm of the cavity is closed by the SESAM.

Each emitter receives its own feedback at a specific light frequency as selected by the grating. The emitter amplifies the light, thereby providing a continuous wave, single-frequency output per emitter. Initially, these frequencies are superimposed in the common arm with random relative phases which generates a random temporal variation of the power. The reflectivity of the SESAM depends on the instantaneous intensity, providing relative-phase de- 


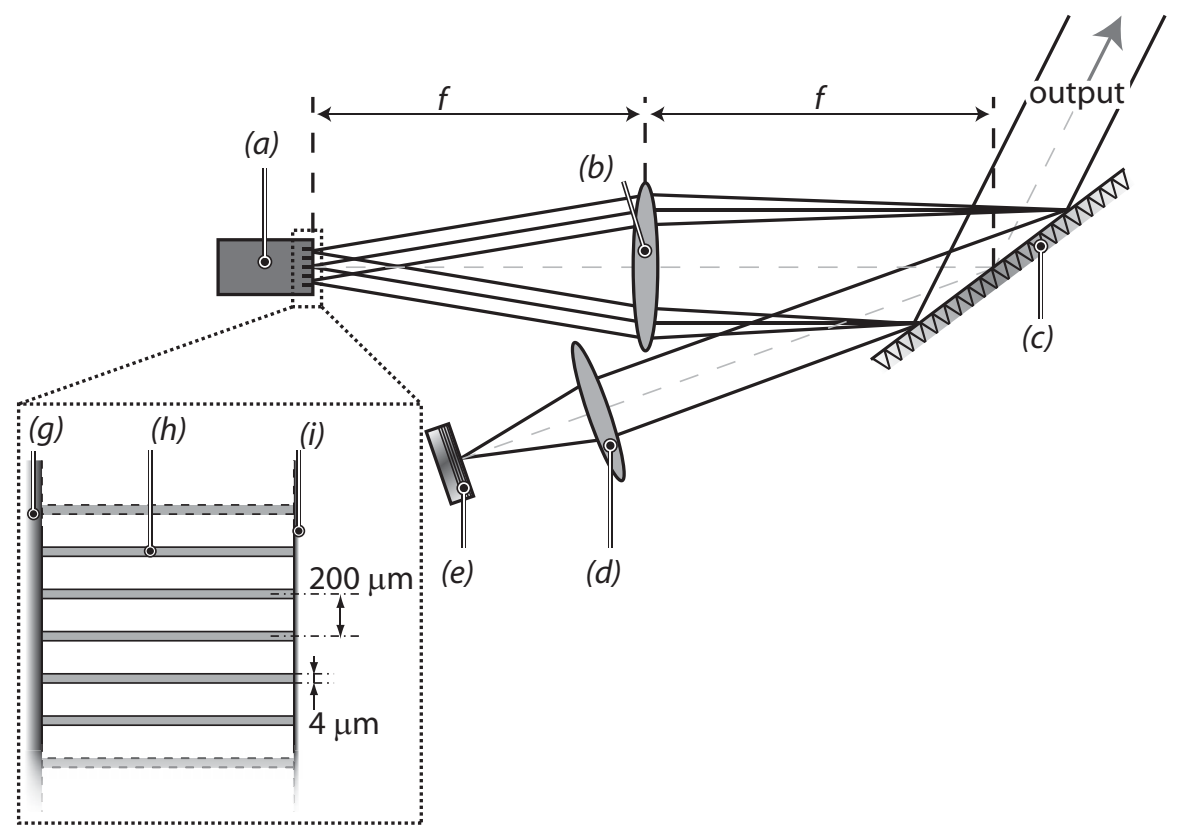

Figure 4.1: (top) Overview. Light originates from the AR coated diode bar (a), and is collimated by a lens (b). The light is combined by a diffraction grating (c), and focused (d) on the SESAM (e). The collimation lens (b) is placed one focal length (f) from the diode bar, and the diffraction grating (c) is one focal length (f) from the lens (b). (bottom left) Magnified view of the diode bar showing several of the emitters. The gain elements (h) are spaced $200 \mu \mathrm{m}$ apart and have a width of $4 \mu \mathrm{m}$. The bar has a high-reflection on the back facet (g) and an anti-reflection coating on the front (i). 
pendent feedback.

Mode locking is initiated by the SESAM becoming saturated, leading to an increased reflectivity when a sufficient number of emitters constructively interfere. This results in increased feedback to the emitters, and creates phase locked side bands on all frequencies through the temporal modulation of the reflectivity, which drives their mutual phase locking. If the frequencies are equally spaced and phases are locked, a regular train of pulses results in the common arm. Note that in the other arm all emitters remain CW emitters, each at its individual, single frequency. The output pulse train can be extracted via the 0th diffraction order of the grating.

Such a SEGA mode locked laser has a number of unusual but beneficial properties. While the phases of the CW lasers are mutually locked by the SESAM feedback, the frequency spacing of the individual CW lasers is determined by the dispersion of the grating and the physical spacing between the emitters. Note that these parameters can be designed to select a desired frequency spacing, and thereby repetition rate, independent of the length of the external cavity. The average power scales with the number of gain elements and is independent of the repetition rate, provided that the peak power remains sufficient to saturate the SESAM. The bandwidth scales as the number of emitters and their frequency separation and can be controlled by switching gain elements on and off. A related approach was suggested in Ref. [63], but in that case the longitudinal modes were not fully separated spatially, so that the gain elements still needed to amplify pulses. Our approach creates pulses by synthesizing a large number of single frequencies [64], without the need for active stabilization.

\subsection{Model}

In the following, an analytical expression is presented that relates the cavity design parameters to the repetition rate and pulse duration. In addition numerical calculations of the laser's temporal dynamics are presented. They are based on amplification and gain saturation in the slowly varying $\mathrm{CW}$ regime for each of the separate gain elements. These calculations provide the requirements for the characteristic SESAM parameters to establish mode locking. The use of a slowly varying CW amplifier model for each of the gain elements is justified by the limited frequency range available to each element, which imposes correspondingly slow dynamics. The dynamics of the SESAM is expected to lie in the ultra-fast regime. To describe the dynamics the phenomenological model developed by Grange et al. [58] is employed.

Due to the complexity of the laser (Fig. 4.1) and its nonlinear dynamics, we employ a numerical model for the theoretical description. A mean field laser model is used, based on a diode bar with a large number of independent, separate gain elements each having a single spatial mode. The parameters in our model are based on a typical single-mode diode laser bar that is commercially 
available (Oclaro, type SPCxxC-980-01). The bar contains 49 emitters separated by $200 \mu \mathrm{m}$, each with a front aperture of $4 \mu \mathrm{m}$ along the slow axis of the emitter. The gain material is InGaAs with a spectral gain bandwidth of $30 \mathrm{~nm}$ around a center wavelength of $975 \mathrm{~nm}$. The maximum specified output power per emitter is $1 \mathrm{~W}$ CW, providing a substantial total output power of $49 \mathrm{~W}$. To relax the constraints on the SESAM's recovery time, the cavity is optimized to provide relatively low repetition rate for this type of laser, while maintaining enough optical power in the cavity for feedback into the diode lasers to generate single frequencies. Taking these considerations into account, we designed a cavity containing a spherical lens $\left(f_{(b)}=300 \mathrm{~mm}, \varnothing 50.8 \mathrm{~mm}\right)$, a 1800 -lines $/ \mathrm{mm}$ diffraction grating, and a SESAM, as depicted in Fig. 4.1. For the modeling of the laser, we do not further specify the focal length of the second lens $(d)$. Instead we vary the fluence to represent different focusing conditions on the SESAM. We account for losses between the emitters and the SESAM (due to scattering, misalignments, non-saturable losses, etc.) in a single loss term of $90 \%$.

\subsubsection{Frequency spacing}

The emitted frequency of the $n^{\text {th }}$ gain element, $\nu_{n}$, can be calculated using the standard expression for the diffraction angles at a grating, modified to take into account the emitter spacing $d$ (also called pitch) and focal distance $\left(f_{(b)}\right)$,

$$
\nu_{n}=\frac{c}{\Lambda_{g}}\left(\frac{1}{\sin \left(\theta_{i}\right)+\sin \left(\theta_{0}-\tan ^{-1}\left(\frac{n d}{f_{(b)}}\right)\right)}\right),
$$

with $n=0$ denoting the gain element located in the center of the diode bar. The total number of elements $N$ equals $2 n+1 . \Lambda_{g}$ is the grating period. $\theta_{i}$ is the angle of diffraction of the -1 st order compared to the normal of the grating. $\theta_{0}$ is the angle of the incident light at the grating originating from the central gain element. $d$ is the physical distance between two adjacent gain elements at the diode bar. $f_{(b)}$ is the distance between the grating and the collimating lens. For further clarification, these constants and variables are also depicted in Fig. 4.2.

Note that Eq.(4.3) is independent the of cavity length. The cavity length of the external cavity does impose a longitudinal mode structure for each separate laser. However, for a longer cavity such as considered here, this spacing (tens to hundreds of $\mathrm{MHz}$ range) is much smaller than the spacing imposed by the grating and emitter spacing (tens to hundreds of $\mathrm{GHz}$ range). Additionally, the considered external grating feedback cavity is rather lossy such that the longitudinal modes of the cavity posses a wider spectral bandwidth than their spacing. This enables the spectrum of the laser bar to become equidistant by mode locking in spite of sightly different cavity lengths of the individual lasers.

A similar argument applies to the following deviation. Eq.(4.3) is weakly dependent on the incident light frequency. This means that an equal spac- 


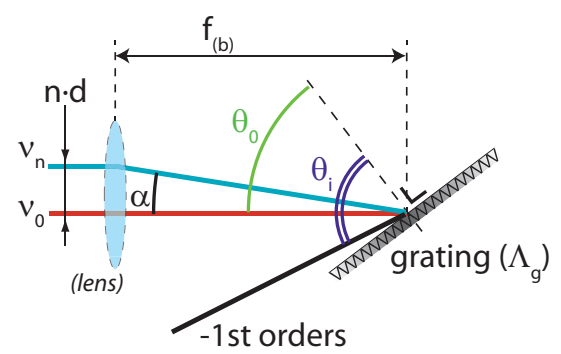

Figure 4.2: Part of the schematic setup as depicted in Fig. 4.1, for clarification of the angles, constants and variables as described in Eq.(4.3). The black line represents the optical path where the beams with different frequencies overlap, while the red and blue line represent the optical paths for different frequencies $\left(\nu_{0}\right.$ and $\left.\nu_{n}\right)$.

ing between the emitters does not yield an equal spacing between adjacent frequencies. The deviation $\delta \nu_{n}$ from the linear frequency spacing is given by $\delta \nu_{n}=\nu_{n}-n \cdot \Delta \nu-\nu_{0}$, which is shown in Fig. 4.3.

Mode locking requires an equidistant comb of light frequencies. $\delta \nu_{n}$ can be compensated for within the angular and spatial acceptance of the gain elements as long as the ratio between the physical size of the emitter and the distance between the emitters (which we call the mark / space ratio) is equal to or exceeds the deviation. In our case the mark / space ratio is $2 \%(4 \mu \mathrm{m} / 200 \mu \mathrm{m})$. Fig. 4.3 shows that the deviation is less than $2 \%$ over a range of 12 emitters, which is indicated by the red line. This means that an equally spaced frequency comb can be achieved over a range of 12 emitters. However, taking the finite numerical aperture of the collimation lens into account $(\mathrm{NA} \approx 0.08)$, the diffraction limited spot size of the light fed back to the diodes has a $\left(1 / e^{2}\right)$ diameter of $7.4 \mu \mathrm{m}$, which is larger than the emitter aperture. This causes an overall loss but also an insensitivity to misalignment and allows equidistant comb elements over a larger number of emitters. For example, if we would consider that only $33 \%$ of modal overlap with the laser aperture is required for lasing, then an equidistant frequency comb extending over a range of 22 emitters seems possible (effective mark / space ratio of $8 \%$ ), as indicated by the green lines in Fig. 4.3. Since diode lasers only need a limited amount of feedback for lasing action (compare Section 2.2.1), an even larger range of emitters might be frequency locked in an equally spaced frequency comb. However, from Eq.(4.3) it also becomes clear that, to use all of the emitters in the mode locking scheme (i.e., to attain an equidistant frequency spacing over the full spectrum), a specific non-equidistant emitter spacing is a prerequisite. This would be achievable by fabricating diode bars with custom emitter spacing. As an alternative, one can also consider to employ an integrated optical waveguide chip. Such a chip would consist of a set of bend waveguides that convert the equidistant pitch of the diode bar to a suitable non-equidistant pitch that yields an equal fre- 


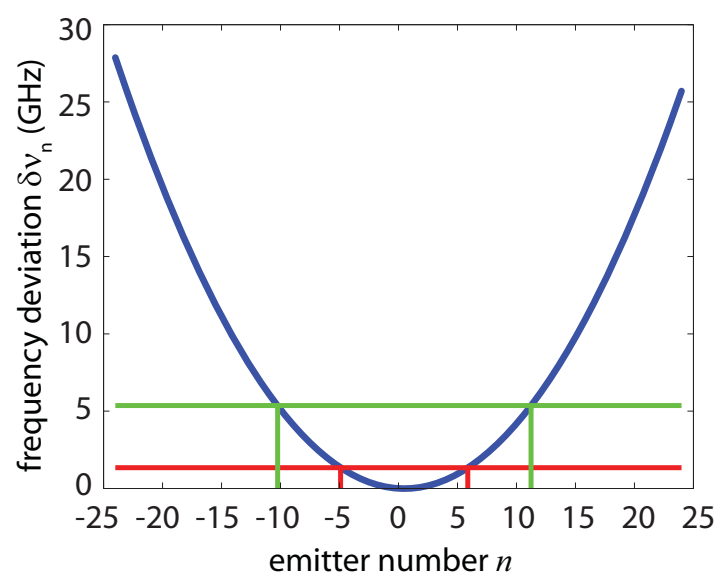

Figure 4.3: The deviation from an equidistant frequency spacing in Eq.(4.3) versus the emitter number $n$. The red lines indicate the number of emitters that would participate in mode locking, assuming that the mark / space ratio of the emitters is the limiting factor. The green lines indicate the number of participating emitters, assuming that minimally a 33\% modal overlap is required to frequency lock the emitters. For these calculations a frequency spacing of $6^{7} \mathrm{GHz}$ is chosen.

quency spacing, following Eq.(4.3). This is called a waveguide pitch converter. In Chapter 5, the design parameters for such a pitch converter are discussed in more detail.

Implementing a specific non-equidistant emitter spacing proved to be a crucial part in the experiments performed on this laser. For the calculations in this chapter, it is assumed that an ideal equidistant frequency comb is possible as based on a sufficient spectral acceptance as described before. In this case Eq.(4.3) can be seen as an expression to design the repetition rate. For instance, a collimating lens with a lower focal distance $f_{(b)}$ will cause a larger frequency spacing and thus yield a higher repetition rate.

While setting a desired repetition rate is possible as described in Eq.(4.3), it turns out that our mode locking method provides a fixed relation between the pulse duration and repetition frequency, as can be seen as follows. The pulse repetition frequency is given by the frequency spacing, $\Delta \nu$, and the Fourierlimited pulse duration is determined by the total bandwidth, $\Delta \nu_{t o t}=N \cdot \Delta \nu$. Therefore, the ratio of the pulse duration and the pulse spacing (called the duty cycle) is fixed and amounts approximately to the inverse number of spaces between the gain elements $(1 /(N-1))$. Thus, if a lens system with an adjustable magnification is inserted between the grating and the gain elements, the repetition rate and pulse duration change simultaneously while their product remains 
constant. To increase the pulse duration independently of the repetition rate, a number of the outer elements could be switched off. For the parameters chosen in our model, the frequency spacing is $67 \mathrm{GHz}$ so that the total bandwidth is $3.3 \mathrm{THz}$, with a block-shaped envelope of the power spectrum. From this a Fourier-limited pulse duration of approximately 300 fs (FWHM) is obtained, with sinc $^{2}$-shaped pulses.

Fig. 4.4 shows the block diagram for our iterative calculation. The field envelope is modeled rather than the oscillating field itself (slowly varying envelope approximation), which is equivalent to shifting the frequency of the center array element to zero. The approximation is justified due to the spectral filtering of light by the grating inside the cavity, which reduces the oscillation of the light field in the single elements to a narrow bandwidth with a correspondingly slow dynamics. The model combines two essential physical processes: the interaction of the light with the SESAM and the amplification of light in the gain elements. Because the reflectivity of the SESAM is time dependent, the SESAM is modeled in the time domain. As, in single elements, singlefrequency light is amplified, the gain is described in the frequency domain. Switching between the frequency and time domain is done by a discrete fast Fourier transform. Both the time and frequency coordinates are represented by a one-dimensional (1D) array of $2^{14}+1=16385$ elements. Based on the physical parameters of the envisioned experimental setup, the frequency spacing between each frequency array element is chosen as $6.7 \mathrm{GHz}$, providing a bandwidth of $110 \mathrm{THz}(16385 \cdot 6.7 \mathrm{GHz})$ over the total array length. This bandwidth provides sufficient resolution $(9.1 \mathrm{fs})$ in the time domain to describe the dynamics of the SESAM (500 fs) and resolve the pulse shape (300 fs). The time array spans $150 \mathrm{ps}$. The center frequencies of the gain elements are placed $67 \mathrm{GHz}$ (ten array elements) apart, so that the total bandwidth covered by the gain elements is $3.3 \mathrm{THz}(49 \cdot 67 \mathrm{GHz})$. Mode locking will result in ten pulses within the time array, all of which are processed in one computational loop. To significantly reduce calculation time, the computational frequency resolution is many times less than the cold-cavity mode spacing of the experimental setup, which is $\sim 150 \mathrm{MHz}$ (assuming a $1 \mathrm{~m}$ total cavity length). This means that in the experimental setup more than 400 pulses occupy the cavity simultaneously, rather than ten as in the calculation.

\subsubsection{Gain model}

A spectral gain profile is used for each gain element to express two issues: 1) the possibility of oscillation on several longitudinal modes per gain element, spaced at $150 \mathrm{MHz}$. 2) The limited spectral filtering imposed by the finite physical size of the emitters in the diode bar and the dispersion by the grating, together with the diffraction limit improved by the finite numerical aperture of the focusing lens $f_{(b)}$ which is calculated to be covering about $600 \mathrm{MHz}$. As a result, approximately five modes can oscillate per gain element. To address the first issue, we allow for five discrete frequencies per element, which we term side 


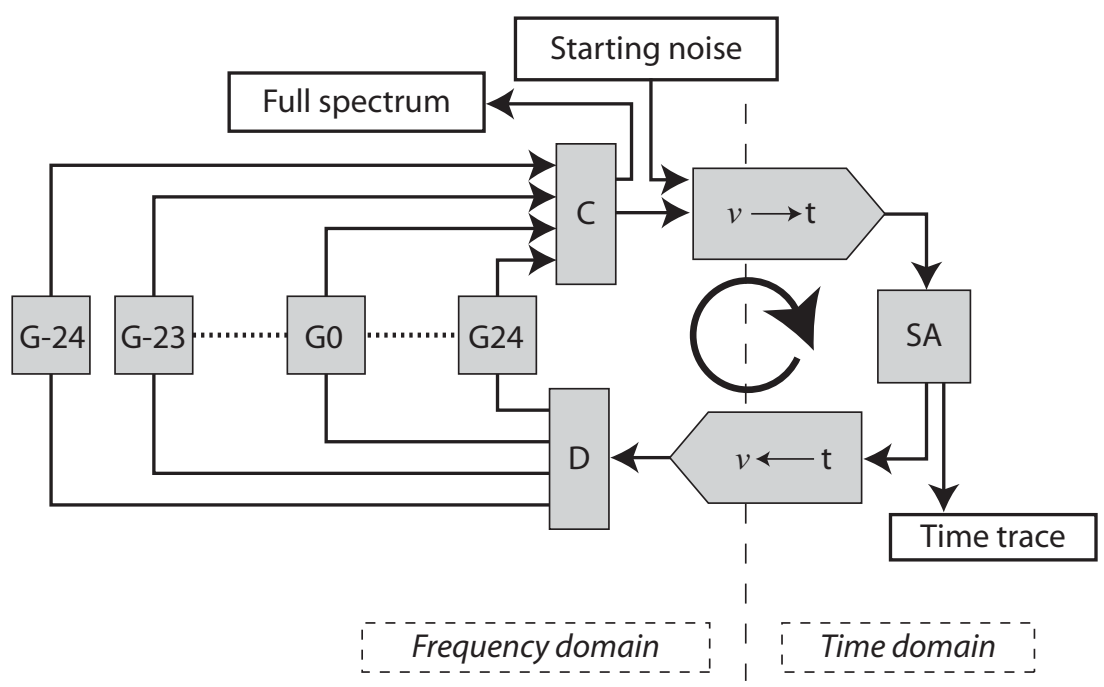

Figure 4.4: Block diagram for the laser model. One iteration consists of light amplification in 49 independent gain elements $(G-24, \ldots, G 24)$, summation of their light-field amplitudes to the full spectrum $(C)$, Fourier transformation to the time domain $(\nu \rightarrow t)$, saturable absorber described in time domain (SA) and monitoring the temporal shape of the output (time trace), inverse Fourier transformation to the frequency domain $(\nu \leftarrow t)$, decomposition into separate spectral field amplitudes (D), and return to the gain elements. Random initial phases are used to model startup of mode locking from noise. 

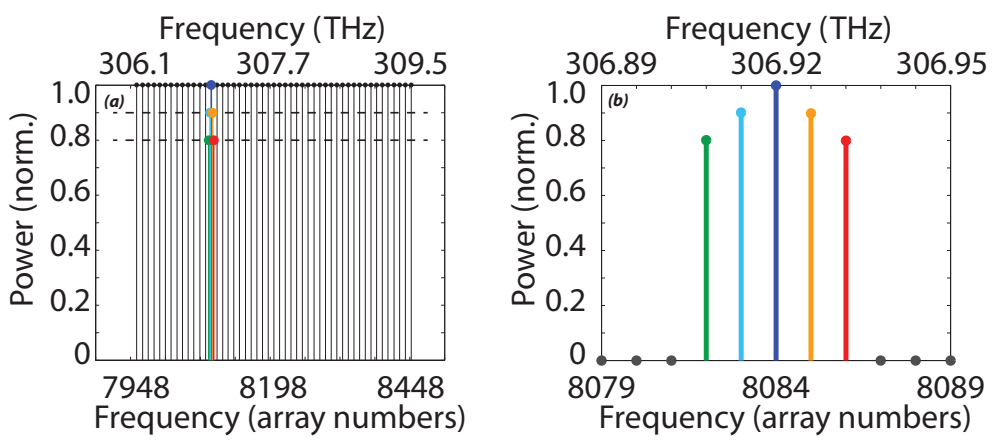

Figure 4.5: Schematic depiction of the modeled gain spectra. (a) shows the overall spectrum, with a block-shaped envelope, indicating equal gain for all emitters. Outside the spectrum (array numbers 17947 and 8449-16385) no gain is present. The dashed lines indicate the power of the side modes at $90 \%$ and $80 \%$. (b) shows a zoom-in of the range of array numbers 8079-8089, which is the modeled gain spectrum of a single emitter.

modes around the center mode (two side modes around each center mode). We recall for clarity that the implementation of the array spacing $(6.7 \mathrm{GHz}$ rather than $150 \mathrm{MHz}$ ) is solely done to reduce calculation time. However, any spacing can be chosen when the injection locking range (Eq.(2.18)) exceeds the cavity mode spacing. This is generally fulfilled for diode lasers with external cavities where both the roundtrip loss and roundtrip gain is very high.

A rough estimate of $90 \%$ loss is used, which includes output coupling, optical waveguide losses in the gain elements [65], losses due to limited apertures, modal mismatch of the feedback at the diode laser facets, collimation errors [66] and the non-saturable losses of the SESAM. To address the second issue, the side modes are given a lower relative gain than the center mode. The spectral gain profile for each gain element was chosen as [0.8 0.91 .00 .90 .8 , which is estimated from the frequency-dependent decrease in spatial overlap of the center mode and the first and second side modes. The gain profile for the individual emitters is shown in Fig. 4.5b.

For the overall shape of the gain spectrum, from the $-n$th to the $n$th center modes, i.e., for the total bandwidth of the modeled laser, a flat gain profile is assumed. This is justified because this bandwidth is substantially smaller than that of the InGaAs gain [67]. The gain profile for the overall spectrum is shown in Fig. 4.5a.

Since we are interested in the dynamics rather than the average power of the laser, the total amount of average power inside the laser is normalized to the known value of $49 \mathrm{~W}$ (1 W per gain element) without reference to the transverse size. This is done by normalizing the saturation intensity $\left(I_{\text {sat }}\right)$ of the gain to the maximum signal intensity inside the gain element $\left(I_{\text {tot }}=1 \mathrm{~W}\right)$. 
Eq.(4.4) than yields $I_{\text {sat }}=1 /\left(g_{0}-1\right)$.

The remaining degree of freedom is the value of the small-signal gain, $g_{0}$, which we choose to be a typical value of 100 over the full length of the gain (i.e., the dimension of $g_{0}$ is $\left[\mathrm{m}^{-1}\right]$ ). The roundtrip losses of $90 \%$ which include all losses as described above, are applied to all the light that is fed back into the individual gain elements before calculating the gain. For the gain of each individual element homogeneous broadening is assumed which implies that, upon amplification, the center mode and the side modes compete for gain. This is approximated as

$$
g\left(I_{\text {tot }}\right)=\frac{g_{0}}{1+I_{\text {tot }} / I_{\text {sat }}},
$$

where $I_{t o t}$ is the sum of intensities of the center and side modes. Eq.(4.4) can be derived from considering the simplified rate equations in steady state, as is shown in Appendix A. The gain in each iteration is calculated using Eq.(4.4) rather than using an integration over the full volume of the gain element. In the initial iterations where steady state is not yet reached, this approximation does not strictly conserve energy per roundtrip and an average output above $49 \mathrm{~W}$ is generated. But, thereafter, saturation reduces the roundtrip gain to a value of 1 for a per-emitter power of $1 \mathrm{~W}$, conserving power over multiple round trips and therefore the energy. Finally, the amplified spectrum is transformed to the time domain using a fast Fourier transform.

\subsubsection{Temporal dynamics}

The reflectivity of the SESAM is described in the time domain. To describe the reflectivity of the SESAM as function of pulse fluence, we follow a phenomenological approach developed by Grange et al. [58],

$$
R\left(\Phi_{P}\right)=R_{n s}-\left(1-e^{-\Phi_{P} / \Phi_{s a t}}\right) \frac{\Delta R}{\Phi_{P} / \Phi_{\text {sat }}}-\frac{\Phi_{P}}{\Phi_{T P A}} .
$$

Here $\Phi_{P}$ is the pulse fluence (for a pulse much shorter than the recovery time $\tau$ ), $\Delta R$ is the modulation depth of the reflectivity, and $R_{n s}$ incorporates the non-saturable losses. Two-photon absorption (TPA) is described using a two-photon fluence $\Phi_{T P A}$, where $\Phi_{T P A}=S_{0}^{2} \Phi_{\text {sat }} / \Delta R[58]$ and $S_{0}=10$, for a typical commercially available SESAM [68]. Two-photon absorption accounts for a decrease in reflectivity with increasing pulse fluence beyond a certain point $\left(S_{0}\right)$. The temporally and spectrally independent cavity losses (including that the non-saturable losses of the SESAM, i.e., $R_{n s}=1$ ) are already included in a single loss and are taken into account in the gain section of the model. To display the typical reflectivity of a SESAM, as based on Eq.(4.5), we have plotted in Fig. $4.6 R\left(\Phi_{P}\right)$ vs. $\Phi_{P} / \Phi_{\text {sat }}$ on a logarithmic scale, for $R_{n s}=1$ and typical values $S_{0}=10$ and $\Delta R=0.2$. This $20 \%$ maximum modulation depth is derived from Eq.(4.5), without the TPA present (dotted line in Fig. 4.6). 


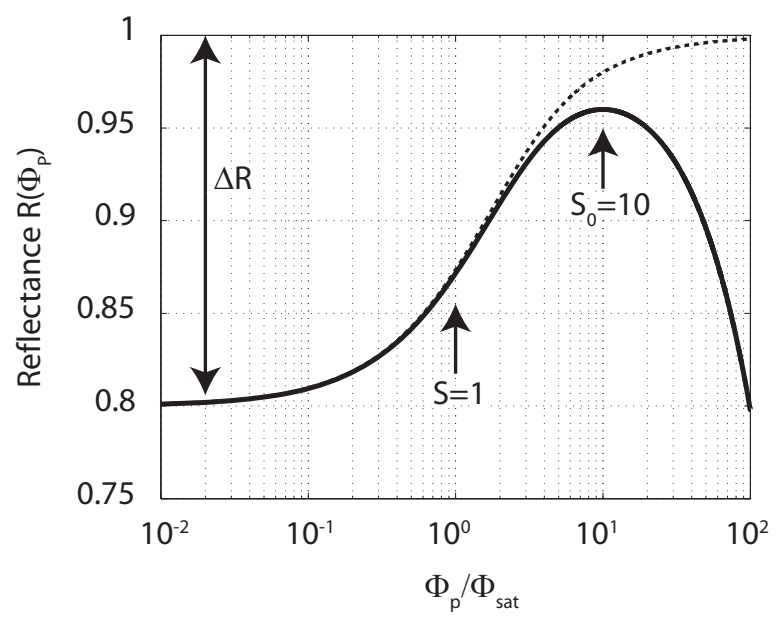

Figure 4.6: Representation of Eq.(4.5), $R\left(\Phi_{P}\right)$ vs. $\Phi_{P} / \Phi_{\text {sat }}$ on a logarithmic scale. For this calculation the values $R_{n s}=1$ and typical SESAM values $S_{0}=10$ and $\Delta R=0.2$ were used. The solid line shows Eq.(4.5) with two-photon absorption (TPA) included, whereas the dotted line shows Eq.(4.5) without the contribution of TPA.

In an experimental setting, the fluence level on the SESAM can be varied continuously by changing the cavity-mode diameter at the SESAM or increasing the pump current through the gain elements. In our calculations the cavitymode diameter is not explicitly specified and the total power is normalized. The pulse energy is related to $1 / 10^{t h}$ of the total power (since there are 10 pulses in the calculation) and the pulse fluence only has relevance with respect to the saturation fluence of the SESAM. To define the effective size of the beam on the SESAM and allow for a pulse energy to be converted to a pulse fluence, we define a parameter $S . S$ is the ratio of the pulse fluence for a Fourier-limited pulse $\left(\Phi_{\text {opt }}\right)$ over the saturation fluence of the $\operatorname{SESAM}\left(\Phi_{\text {sat }}\right): S=\Phi_{\text {opt }} / \Phi_{\text {sat }}$. The fluence values in Eq.(4.5) are pulse fluences for pulses much shorter than the recovery time and therefore have no explicit time dependence. The fluences correspond to the saturation level in the SESAM within a time interval too short to experience the exponential decay of the saturation over the recovery time $\tau$. To assign to a time-dependent intensity, $I(t)$ as used in the model, the appropriate fluence as defined in Eq.(4.5), by taking account the dynamic recovery of the absorption, we use

$$
\Phi_{P}=\int I(t) \exp (-t / \tau) d t
$$

where $\Phi_{P}$ is the fluence and where $\tau$ is the time constant for recovery of the absorption (recovery time). It should be noted that, due to the weighting of the intensity with an exponential recovery of absorption in the SESAM, the pulse 
fluence $\Phi_{P}$ can only be applied when used in combination with the SESAM's recovery time and does not describe the pulse energy directly.

In the numerical modeling, the integration is performed as a running sum and the resulting value for $\Phi_{P}$ is inserted into Eq.(4.5) to obtain the time dependent intensity reflectivity, $R\left(\Phi_{P}\right)$. The time-domain field amplitude is multiplied by the square root of $R\left(\Phi_{P}\right)$. This concludes the time-domain portion of the calculation iteration and the time-domain field amplitude is transformed by a fast Fourier transform (FFT) to the frequency domain to begin a new iteration.

\subsubsection{Initial conditions and evaluation}

The calculations are started with a power of $1 \mathrm{~W}$ per gain element divided over the main mode and the side modes in the shape of the spectral gain profile as mentioned before. To model the influence of spontaneous emission on the onset of mode locking, the phases of the modes are assigned new random values at the beginning of each simulation. For all the data reported here, the simulations were terminated at 500 iterations because it was observed that a steady state was generally reached within 500 iterations. To observe the impact of the initial phases on mode locking, each simulation is run multiple times with the same SESAM parameters.

To identify for which set of SESAM parameters mode locking occurs, simulations were performed for a wide range of the SESAM parameters $S, \tau$, and $\Delta R$. To evaluate the numerous time traces, it is useful to define a criterion for successful mode locking by comparing each calculated time trace with that of a Fourier-limited pulse train that possesses the maximum possible peak power. Successful mode locking was defined as the occurrence of any peak power that exceeds half of this maximum possible peak power. With this criterion the probability of mode locking is obtained by determining how many times, out of a given number of simulations with identical parameters, successful mode locking was observed. The results showed that ten runs are sufficient to identify a parameter range where mode locking occurs, called the mode locking regime, with a distinguishable boundary to parameters with which no mode locking occurs. The probability of mode locking is defined as the number of times mode locking was observed, divided by the number of simulations per parameter set (which is chosen to be ten).

\subsection{Results}

Based on the boundary conditions in which Eq.(4.5) is valid, we expect to see mode locking for values of $S$ above $S=1$, where the pulse fluence equals the SESAM's saturation fluence. At $S=10$ we expect mode locking over the largest range of SESAM parameters, $\Delta R$ and $\tau$. For higher values of $S$, however, twophoton absorption is expected to decrease the mode locking regime. Results 
for $S: 0.1,1,3,5,10,20$, and 50 are shown in Fig. 4.10. The values for the SESAM's recovery time and modulation depth are chosen similar to what currently commercially available SESAMs provide [68]: $\Delta R$ is chosen to be between $5 \%$ and $40 \%$, in steps of $5 \%$, and $\tau$ is chosen between 0.5 ps and 10 ps.

To show how the pulse train and spectrum evolve, three different iterations are displayed in Fig. 4.7-4.9. For these calculations the SESAM parameters were chosen at the edge of the mode locking regime $(\Delta R=40 \%, \tau=3 p s$ and $S=3$ ), to have a clear evolution of the pulses over a large range of the 500 iterations. Fig. 4.7 shows the spectral power and corresponding time trace, after one iteration, Fig. 4.8 after 100 iterations, and Fig. 4.9 after 500 iterations. The insets in Fig. 4.7a, Fig. 4.8a and Fig. 4.9a show the spectrum including all side modes over the range of the gain, whereas Fig. 4.7a, Fig. 4.8a and Fig. 4.9a show a zoom-in of the spectrum to ten array numbers (array numbers 8079-8089, the distance between two neighboring center modes). The dots (and vertical lines) show the calculated power of the light at these frequencies. The insets in Fig. 4.7b, Fig. 4.8b and Fig. 4.9b show the full time trace of our calculations, whereas Fig. 4.7b and Fig. 4.8b show a zoom-in over 2000 array numbers. Fig. $4.9 \mathrm{~b}$ shows a zoom-in over only 400 array numbers to better resolve the pulse shape at the end of the calculation. 

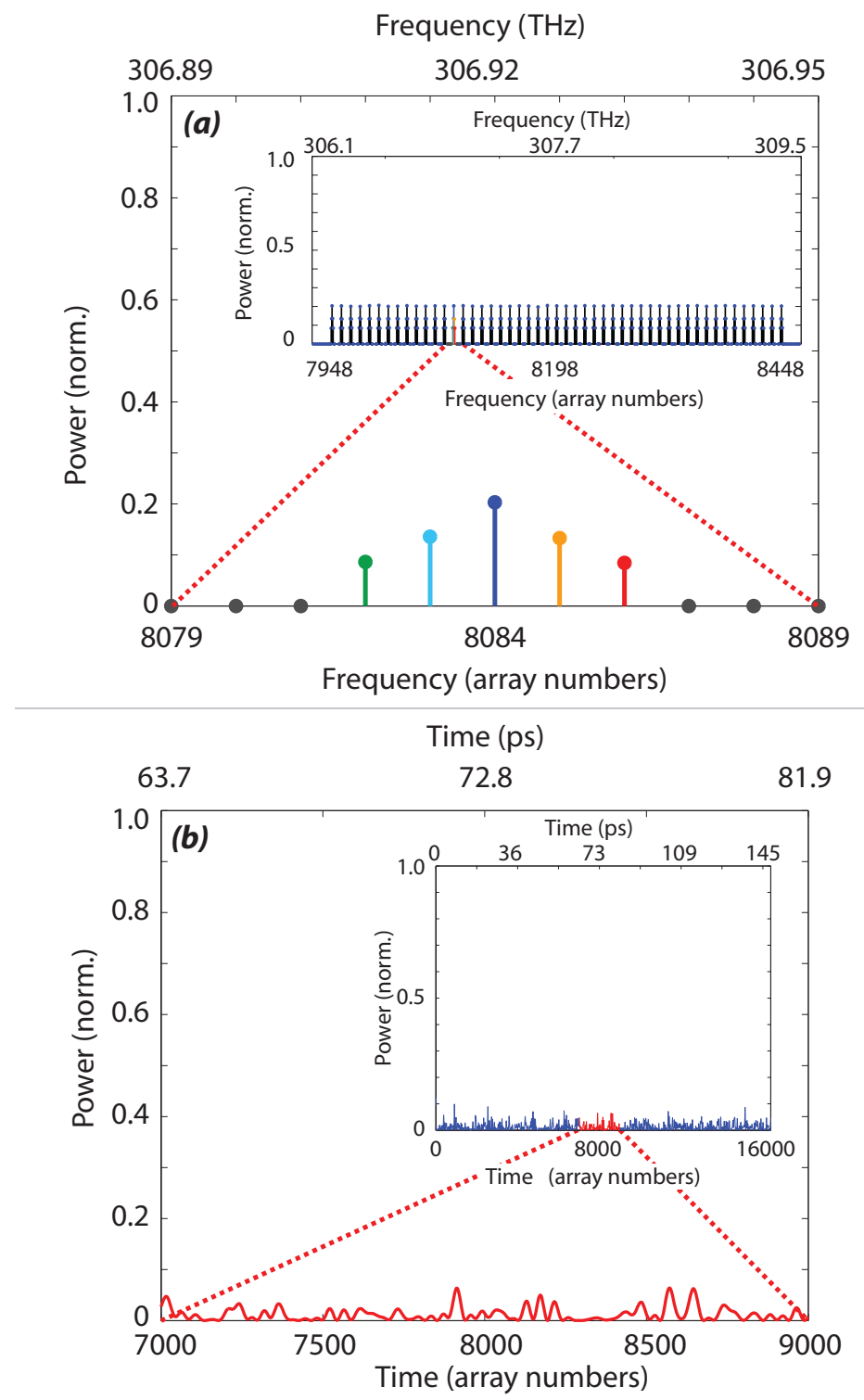

Figure 4.7: Calculation results after 1 iteration, for $\Delta R=40 \%$, $\tau=3 \mathrm{ps}$ and $S=3$. (a) shows the power spectrum, with (b) the corresponding time trace. All power spectra are normalized to the maximum power for $1 \mathrm{~W}$ per emitter. The time traces are normalized to the Fourier-limited maximum peak power. The laser starts with multiple frequencies per gain element and a random phase spectrum. 

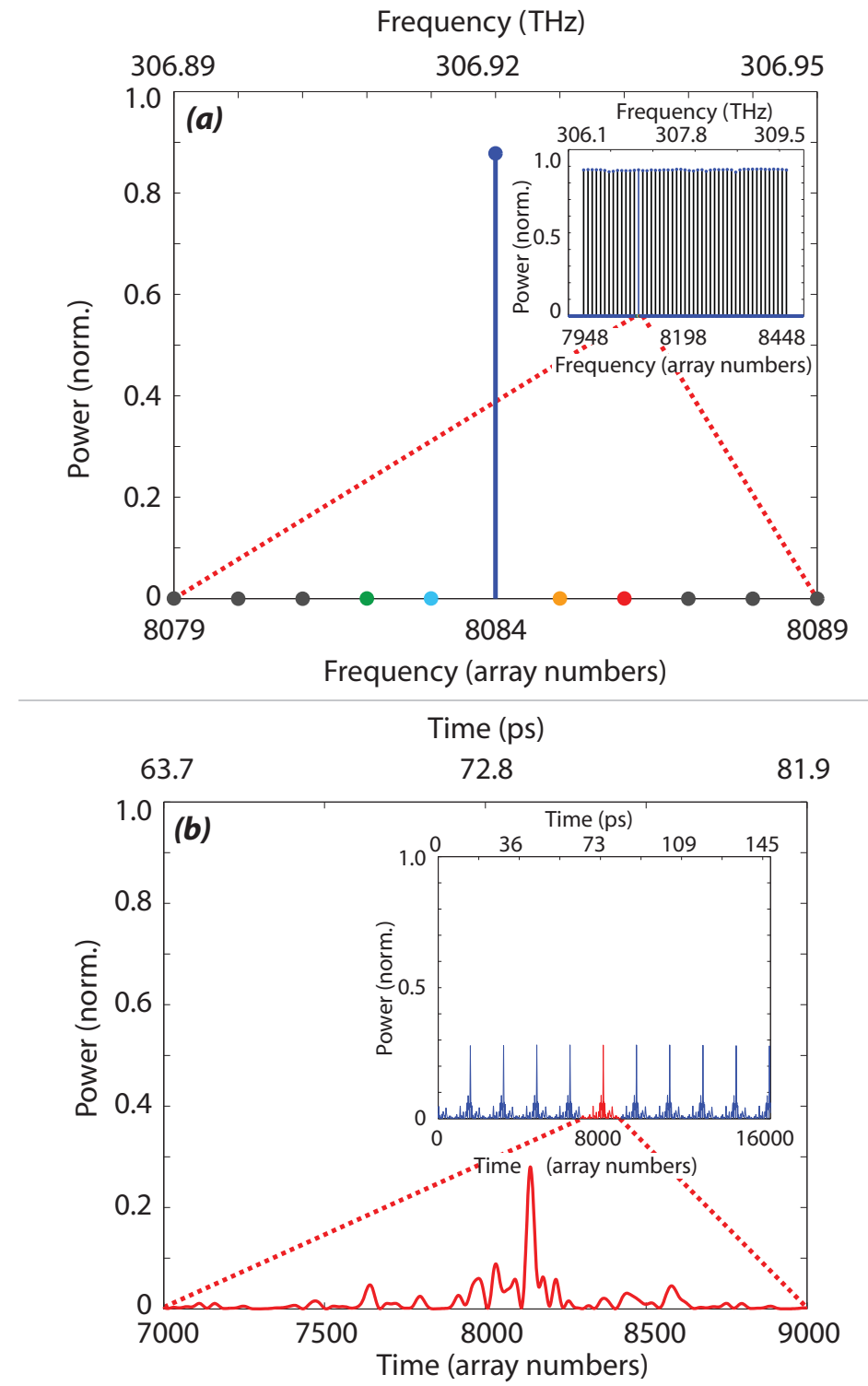

Figure 4.8: Calculation results after 100 iterations, for $\Delta R=40 \%$, $\tau=3 \mathrm{ps}$ and $S=3$. (a) shows the power spectrum, with (b) the corresponding time trace. 

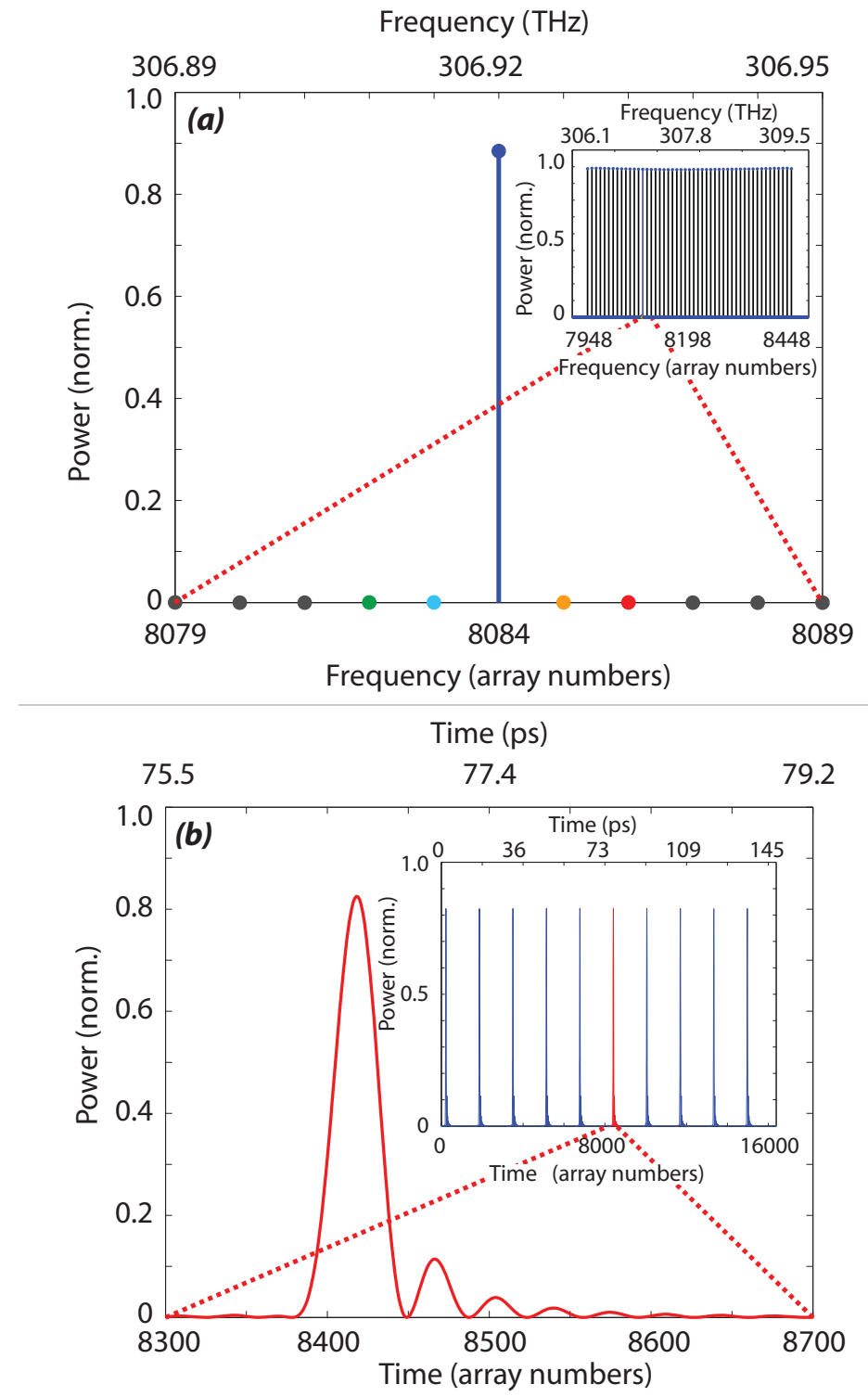

Figure 4.9: Calculation results after 500 iterations, for $\Delta R=40 \%$, $\tau=3 \mathrm{ps}$ and $S=3$. (a) shows the power spectrum, with (b) the corresponding time trace. 
In Fig. 4.7a, after one iteration, it can be seen that all side modes are still present at the spectrum. However, their power relative to the center frequency is lower than at the start of the calculation, due to gain competition and a lower relative gain. After 100 iterations, as can be seen in Fig. 4.8a, no significant power is observed for the side modes anymore and all of the available power is present in the center frequencies. Furthermore, the power at the different gain elements is not precisely equal anymore (the envelope over the full laser spectrum is not entirely smooth; the largest difference in height between the adjacent frequencies is $1.6 \%$, while the average is $0.3 \%$ ). We expect that this is due to the phase dependent exchange of energy between the modes caused by sideband generation in the SESAM. After 500 iterations (Fig. 4.9a), the envelope is smooth, which is an indication for mode locking [69]. This is quantified by the largest difference in height between the adjacent frequencies is $0.23 \%$, while the average is $0.05 \%$, showing that the spectrum in Fig. $4.9 \mathrm{a}$ is significantly smoother than in Fig. 4.8a. The spectrum has an almost square-shaped envelope, which is expected because the spatial separation of the gain for the 49 light frequencies acts as an inhomogeneous broadening of the gain. In the time trace after one iteration (Fig. 4.7a), only a randomly fluctuating power is observed, indicating that all the laser modes oscillate with a random phase. After 100 iterations (Fig. 4.8b), the initial stages of a pulse train can be observed, with irregularly shaped pulses, at a regular interval. This shows that, in this state, not all phases in the spectrum are locked, and, as a result, the peak power is still rather low. After 500 iterations (Fig. 4.9b), short, almost Fourier-limited, pulses are observed, with a FWHM pulse duration of 273 fs (i.e., 30 array numbers).

The remaining deviation from the Fourier limit is mainly due to the asymmetric shape of the pulses. The asymmetry can be explained by the fluencedependent reflection of the SESAM that preferentially absorbs the leading edge of the pulses. This preferential absorption of the front also induces a slow shift (6.5 fs per iteration) of the pulse as the iterations progress. The side pulses, seen only behind the main pulse, are due to the shape of the laser's overall spectrum. A square-shaped spectrum, in the Fourier limit, would provide sinc ${ }^{2}$-shaped pulses, with side pulses.

The most important conclusion from the results in Fig. 4.7-Fig. 4.9 is that our novel approach to mode locking appears promising for an experimental demonstration since the calculations are based on realistic parameters, i.e., taken from the specifications of commercially available components, particularly with regard to the parameters of the SESAM.

We investigated the probability of mode locking, as described above, for $0.1<S<50,5 \%<\Delta R<40 \%$ and 0.2 ps $<\tau<10$ ps. Fig. 4.10 summarizes the observed mode locking probability in graphs with pair-wise variation of $\tau$ and $\Delta R$ for various values of $S$. Black indicates a mode locking probability of unity while mode locking was observed less often for lighter shades of gray. The small crosses in the figure represent the calculations in which mode locking was not observed. It is generally found that for low values of $S$ (corresponding to 

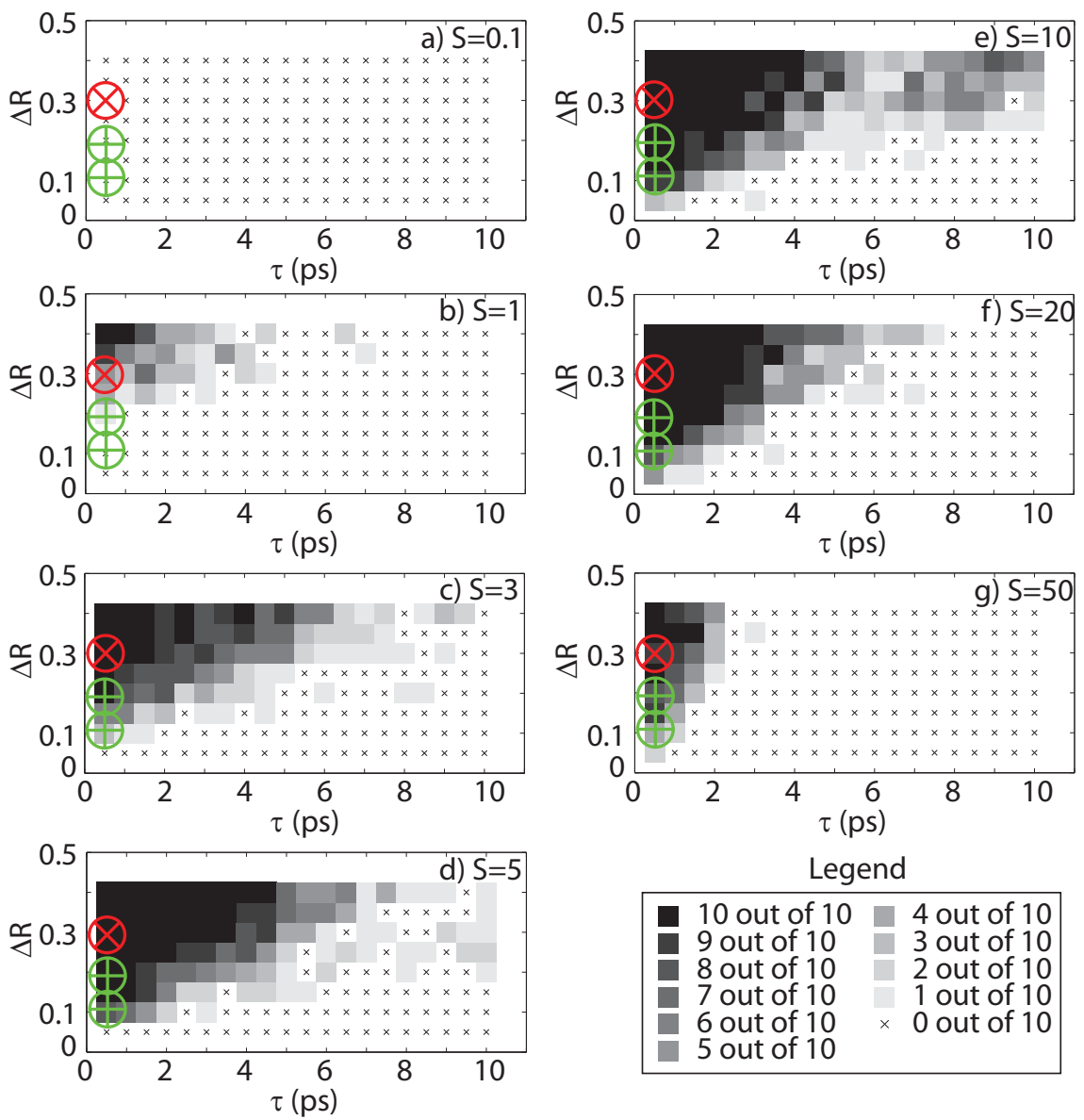

Legend

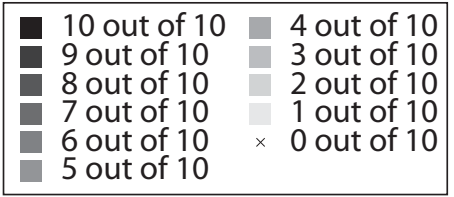

Figure 4.10: Probability of mode locking as a function of $\Delta R$ and $\tau$ for (a) $S=0.1$, (b) $S=1$, (c) $S=3$, (d) $S=5$, (e) $S=10$, (f) $S=$ 20 and $(g) S=50$. Darker shades represent areas where mode locking was observed more often. $\otimes$ indicates the most suitable SESAM for our laser that is also commercially available at this time, i.e., the $S E S A M$ with the highest $\Delta R$ and the lowest $\tau$. For comparison, the second and third best available SESAMs are indicated by the $\oplus$ [68]. 
weaker focusing on the SESAM or a lower saturation fluence), mode locking is only observed for fast absorbers (small $\tau$ ) and absorbers with a high modulation depth (large $\Delta R$ ). This can be understood, because, in Eq.(4.5) for small values of $S$, the SESAM's reflectivity shows a modulation of less than $\Delta R$, making the loss differential too small to induce mode locking.

As $S$ increases with sharper focusing on the SESAM, so does the parameter range for which mode locking successfully occurs. This is the case because, for larger $S$, the modulation due to saturation increases, which relaxes the requirements for the modulation depth and response time. However, it can be seen in Fig. 4.10f and g that a further increased fluence in the SESAM $(S=20$ and $S=50$, respectively) narrows the mode locking regime for $\Delta R$ and $\tau$ again. This can be understood, because in Eq.(4.5) for values of $S>10$, twophoton absorption becomes significant enough to reduce the modulation of the reflectivity. In general, selecting a larger $\Delta R$ means that the same reflectivity change, and thus the same effect on the laser dynamics, is reached for a lower fluence, thereby broadening the operating regime for which mode locking is observed. Similarly, a short $\tau$ ensures a stronger differential gain for the pulsed state which improves the mode locking.

Current SESAMs are optimized for the standard approach to mode locking, i.e., for rather low (tens of $\mathrm{MHz}$ ) repetition rates, so that longer recovery times, in the order of a several ns, are sufficient to induce mode locking. In our approach, the pulse repetition frequencies can be much higher, such as $67 \mathrm{GHz}$ (pulse spacing $15 \mathrm{ps)}$ ) for the laser described here, and significantly shorter recovery times are required. That these can indeed be obtained, although at the expense of a somewhat reduced modulation depth, can be seen in Fig. 4.10. There, the best commercially available SESAMs [68] that could be identified at this moment for the described laser, are indicated with symbols $(\otimes$ and $\oplus)$, with a recovery time of about $\tau>0.5 \mathrm{ps}$ and with modulation depths, $\Delta R$, between $15 \%$ and $30 \%$. The calculations show that such values should suffice for mode locking if a sufficiently high fluence of the incident pulses, about $S=10$, can be realized in spite of the high repetition rate. If a mode area at the SESAM of $50 \mu \mathrm{m}^{2}$ is assumed, together with a typical SESAM value for $\Phi_{\text {sat }}$ of $60 \mu \mathrm{J} / \mathrm{cm}^{2}$, we conclude that it would be possible to achieve $S=10$ with a total power of $20 \mathrm{~W}$ in the common arm. This power is below the specifications of the diode bar that is considered here. This estimate, thus, indicates that our novel mode locking scheme should be feasible with currently available components.

\subsection{Conclusions}

The modeling that has been given here, reveals the influence of the physical properties of the semiconductor saturable absorber mirror (SESAM) on the feasibility of separate gain (SEGA) mode locking, at a specific example of a diode laser bar. The ongoing development of faster SESAMs with a higher 
modulation depth [14] makes it realistic to assume that mode locking over a wide range of average powers and repetition rates is achievable. Our approach to mode locking lifts current limitations on the combination of repetition rate and output power. Although we have used our model to analyze mode locking of a specific laser, which is a diode laser bar with $49 \mathrm{~W}$ average output power, $67 \mathrm{GHz}$ repetition rate and $300 \mathrm{fs}$ pulse duration, the model can also be applied to investigate separate gain mode locking requirements for other types of laser or at different combinations of output power, repetition rate and pulse duration. We have taken the theoretical considerations as described in this chapter as the basis for a first step of an experimental investigation of SEGA mode locking. The experimental setup, though only leading to indecisive conclusions so far, and results will be described in Chapter 5 . 


\section{5 \\ SEGA mode locking experiments}

\subsection{Introduction}

In this chapter, we present experimental investigations of a new type of laser setup intended for the demonstration of separate gain (SEGA) mode locking as was theoretically investigated in the previous chapter. Two different setups will be described which we have tentatively called the initial and improved setup. Additionally, the results of the experiments performed with these respective setups are described. Because the experiments are still ongoing, we have chosen to present the results in a chronological manner.

First we will discuss the details of the initial setup in Section 5.2. We have performed experiments with this setup using a semiconductor saturable absorber mirror (SESAM) selected for maximum available speed and contrast (see Chapter 4). To understand the interpretation of the performed measurements, we describe the used measurement methods in Section 5.3. The experiments as described in Section 5.4 focus on two types of measurements: temporal and spectral measurements. To look for evidence of pulses, the temporal measurements are performed using an intensity autocorrelator. To look for an equidistant comb of light frequencies (frequency comb) spectral measurements are performed using an optical spectrum analyzer. Based on the results from these experiments, several issues were identified that inhibits the possibility of stable and reproducible mode locking. The foremost difficulty was already discussed in the previous chapter, Section 4.4.1, regarding the requirement of achieving an equidistant frequency comb. The experimental spectral and temporal observations are given in Section 5.4. To solve the possibly underlying issues with an insufficient equidistance of frequencies we present the 


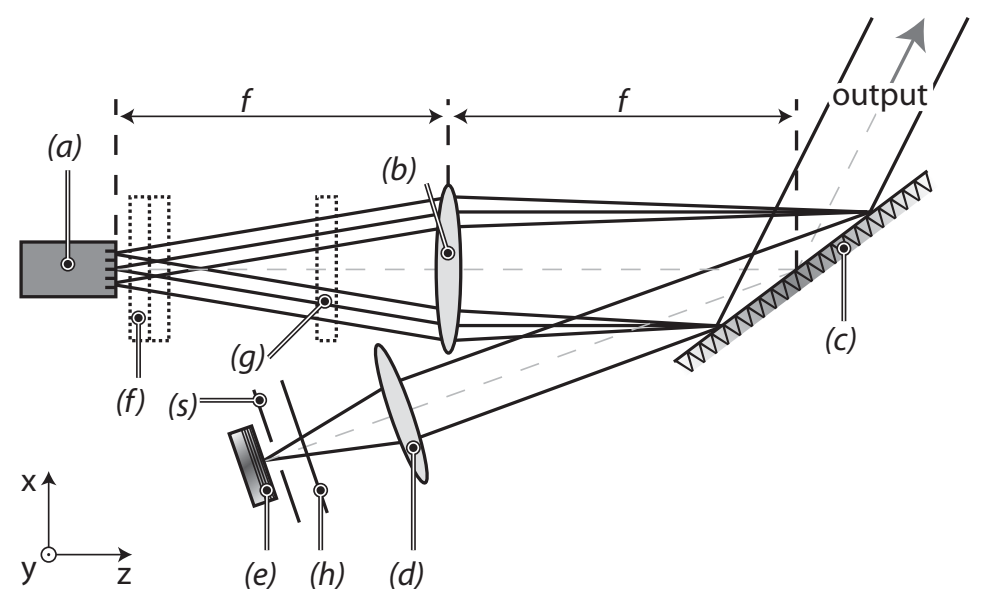

Figure 5.1: Overview of the experimental setup of the SEGA mode locked laser. Light originates from the AR coated diode bar (a). Light diverging in the $x$-direction is collimated by a spherical lens (b), while light diverging in the $y$-direction is collimated by a set of cylindrical lenses (f) and ( $\mathrm{g}$ ) and the spherical lens $\mathrm{b}$. The light is combined by a diffraction grating (c), and focused by a lens (d) on the SESAM (e). The collimation lens (b) is placed one focal length $f_{(b)}$ from the diode bar, and the diffraction grating (c) is one focal length $f_{(b)}$ from the lens (b). The output of the laser can be changed by rotating a $\lambda / 4$ plate (h). An adjustable mechanical slit ( $\mathrm{s})$ is placed in front of the SESAM.

improved setup in Section 5.5. There we describe the incorporation of an array of non-equidistant waveguides, called a pitch converter. The implementation and advantages of the improved setup will also be addressed there. Again, temporal and spectral measurements are performed, as described in Section 5.6. The interpretation of these measurement results was not straightforward. Some measurements seemed to indicate a frequency comb with random phases, while other measurements are better explained assuming some signs of mode locking, as will be summarized and discussed in Section 5.7.

\subsection{Initial setup}

The setup as presented in Chapter 4 forms the basis for the experimental work described in this chapter. The setup is depicted in more detail in Fig. 5.1.

Let us recall from Section 4.4.1 that in this setup, at least principally, the frequency spacing of the frequency comb and hence the expected pulse repetition rate can be freely chosen with an appropriate focal distance of the lens (b), and with a suitable period of the diffraction grating (c). For a first 
experimental approach aiming on a demonstration, however, a low repetition rate of the laser is preferred, in order to enable the used SESAM, in spite of a finite recovery time, to dominate the phase dynamics of the oscillating modes. An accordingly lower frequency spacing can be achieved via a large focal distance $f_{(b)}$ and a small grating period $\Lambda$. This has, however, the disadvantage of introducing more loss to the cavity. First, the diameter of the lens can become too small to capture and collimate all the light from the diode bar. Increasing the diameter of the lens is not a viable option, because the physical size of the diffraction grating is technically limited due to fabrication issues. Secondly, light that has passed the rest of the cavity and is reflected back towards the diode bar will be focused onto the individual emitters by lens $b$. However, the focal spot will have a modal mismatch with the diode laser's mode radius. A larger focal distance of the lens will increase the focal spot and thus increase the loss due to the modal mismatch. Normally, this could be compensated by increasing the beam size before being focused by the lens. However, this is not possible, because the physical limitations of the size of the grating restricts the maximum size of the beam. The repetition rate can also be decreased by choosing a smaller grating period. However, to let the diffraction angle for the first order not exceed $90^{\circ}$ (with regard to the grating normal), the grating period is not allowed to be smaller than half of the used wavelength. In our case the central wavelength of the diode laser is $\lambda_{0}=975 \mathrm{~nm}$, i.e., the smallest grating period is about $488 \mathrm{~nm}$. In view of the named restrictions we decided to choose a configuration with $f_{(b)}=300 \mathrm{~mm}$ and a grating with 1800 lines $/ \mathrm{mm}$. Recalling that the spacing between the emitters in the diode bar is $200 \mu \mathrm{m}$ and using Eq.(4.3) with the named lens and grating parameters, we calculate that the repetition rate for this setup is $67 \mathrm{GHz}$. To collimate the light in the other transverse beam direction, $y$, we chose the values of the focal distances of the two cylindrical lenses $(f)$ as $f_{f 1}=20 \mathrm{~mm}$ and $f_{f 2}=30 \mathrm{~mm}$ and the cylindrical lens $(g)$ as $f_{g}=-200 \mathrm{~mm}$.

To be able to continuously vary the output coupling of the laser, a $\lambda / 4$ plate is added in the common arm. Rotating the $\lambda / 4$ plate changes the polarization of the light returning to the grating. Because the grating diffracts light with p-polarization more efficiently than light with s-polarization, changing the polarization in the common arm will increase the light in the $0^{\text {th }}$ order, i.e., the output of the laser.

In the common arm, the lens $(d)$ has a focal length of $f_{(d)}=150 \mathrm{~mm}$. In the named configuration, the measured size of the beam, before passing through lens $(d)$, is $12 \mathrm{~mm}$ in the $\mathrm{x}$-direction and $10 \mathrm{~mm}$ in the $\mathrm{y}$-direction with a rectangular shape. Approximating this with a diffraction limited elliptic shape for the focal spot, the area of the focal spot is $2 \cdot 3 \cdot 10^{-6} \mathrm{~cm}^{2}$. To restrict the number of possible angles with respect to the grating at which the frequencies in the common arm can oscillate, i.e., to increase the spectral resolution of the grating feedback, an adjustable mechanical slit $(s)$ is added at a distance of $1 \mathrm{~cm}$ in front of the SESAM.

According to its specifications, the SESAM (e) selected for the experiments 
and placed in the common arm has a maximum modulation depth of $\Delta R=30 \%$, a recovery time $\tau=500 \mathrm{fs}$ and a saturation fluence $\Phi_{\text {sat }}=60 \mu \mathrm{J} / \mathrm{cm}^{2}$. In order to reach saturation fluence with the described beam and lens $(d)$ at a repetition rate of $67 \mathrm{GHz}$, we calculate that an average power of $9.2 \mathrm{~W}$ is required.

In the previous chapter, we have addressed the difficulty of achieving perfectly equidistant spacing of the laser frequencies with evenly spaced diode emitters. This is due to the nonlinearity of the diffraction law that describes the dispersion of the grating, see Eq.(4.3), when subtending a large number of emitters. When subtending a smaller amount of emitters and thereby a smaller angular range, the nonlinearity of Eq.(4.3) becomes reduced. To restrict the number of emitters experimentally, i.e., the number of modes that can oscillate, we have added a second adjustable mechanical slit in front of the diode bar. In the experiments, we chose to restrict the number of modes to 26 . The required power of $9.2 \mathrm{~W}$ in the common arm was achieved with 26 modes when operating the diode bar with a pump current of $\mathrm{I}_{L D}=26 \mathrm{~A}$.

\subsection{Measurement methods}

To motivate the type of measurements performed, we recall that the expected basic signatures that would prove a successful SEGA mode locking are a regular, strictly periodic train of ultrashort pulses that spectrally corresponds to a precisely equidistant comb of light frequencies. To measure the presence of such a comb of light frequencies (frequency comb) we use an optical spectrum analyzer (OSA, ANDO AQ6317), set to a resolution of $0.01 \mathrm{~nm}(3.2 \mathrm{GHz})$. In the spectral measurement we expect to observe an equidistant frequency comb with a frequency spacing, $\Delta \nu$, in the order of $67 \mathrm{GHz}$ if the described grating feedback works properly.

To look for evidence of pulses, the temporal measurements are performed. If it were possible to directly measure the intensity of ultrashort pulses vs. time, one would expect as the laser output a train of pulses with a duration of several hundred fs, and temporally spaced by the inverse mode spacing, $\tau=1 / \Delta \nu$, which is about 15 ps. However, such direct measurement is usually not possible, due to the limited temporal response of photodetectors. To nevertheless investigate the temporal structure of the laser output, we applied intensity autocorrelation, although this yields only a temporally average information and a significant background. In our experiments we use a modified intensity autocorrelator (Inrad model 5-14B). The autocorrelator is equipped with a nonlinear crystal that generates the second harmonic of the infrared laser input. Second harmonic generation (SHG) is achieved with collinear type I phase matching. We chose for a collinear rather than a non-collinear alignment because of the relatively low peak power of the expected pulses, causing a relatively low conversion of the infrared laser input into its second harmonic. A collinear alignment provides a longer interaction length of the infrared beam with the SHG crystal and thus a higher second harmonic intensity is generated. 
However, specifically for our experiment, collinear intensity autocorrelation can yield a second harmonic output vs. delay that is less straight forward to interpret.

To illustrate more clearly how intensity autocorrelation (IA) traces are to be interpreted and where the limits of this technique are, we briefly recall the operation principle of the used autocorrelator.

In the autocorrelator, the incident light field is split into two parts of equal amplitude, E, by a beam splitter and sent into two arms. The two arms are delayed with respect to each other, with a repeatedly swept delay spanning a range of 119 ps. Repetitive sweeping with $26.5 \mathrm{~Hz}$ is achieved by means of rotating glass blocks. The point of equal arm length (zero delay) can be manually tuned, over a range of $133 \mathrm{ps}$. The light of the two arms is superimposed again at the beam splitter and focused collinearly into a $1 \mathrm{~mm}$ thick BBO SHG crystal. The second harmonic output is detected by a photomultiplier tube which is not sensitive for the fundamental wavelength. The expected second harmonic intensity, apart from a constant factor, is then described by correlating the electric fields as $[70,71]$

$$
I_{A C}\left(t^{\prime}\right)=\int_{-\infty}^{\infty}\left|\left(E(t)+E\left(t-t^{\prime}\right)\right)^{2}\right|^{2} d t,
$$

where $E(t)$ is the electric field of the light from one arm in the autocorrelator, and $E\left(t-t^{\prime}\right)$ is the electric field of the light from the other arm with delay time $t^{\prime}$. Evaluation of the integral yields

$$
\begin{aligned}
I_{A C}\left(t^{\prime}\right) & =\int_{-\infty}^{\infty} I^{2}(t)+I^{2}\left(t-t^{\prime}\right) d t \\
& +4 \int_{-\infty}^{\infty} I(t) I\left(t-t^{\prime}\right) d t \\
& +4 \int_{-\infty}^{\infty}\left(I(t)+I\left(t-t^{\prime}\right)\right) \operatorname{Re}\left\{E(t) E^{*}\left(t-t^{\prime}\right)\right\} d t \\
& +2 \int_{-\infty}^{\infty} \operatorname{Re}\left\{E^{2}(t) E^{* 2}\left(t-t^{\prime}\right)\right\} d t,
\end{aligned}
$$

where we have used $|E(t)|^{2}=I(t)$ and $\left|E\left(t-t^{\prime}\right)\right|^{2}=I\left(t-t^{\prime}\right)$.

The first integral in Eq.(5.2) contributes a constant background signal, the second integral yields the desired autocorrelation of the intensity. The last two integrals remain dependent on the autocorrelation of the field, i.e., they describe interference fringes. Upon scanning the arm length difference, if the detector is too slow to detect the rapidly changing interference fringe, as is the case in our autocorrelator, these two terms are averaged out by the detector such that they can be neglected [70]. Upon closer investigation, Eqs.(5.1) and (5.2) predict that for a pulse, regardless of the pulse shape, a maximum peak to background ratio of $3: 1$ would be observable. 
In an experiment, however, this is not generally the case, because certain assumptions which the derivation of Eqs.(5.1) and (5.2) are based upon are not exactly fulfilled. The main assumption is that the power returning from the two arms into the SHG crystal is exactly equal, and that the beams from the two arms are in perfect spatial overlap. The inspection of our autocorrelator yielded that the delayed arm carried approximately twice the power of the other arm, which, according to Eq.(5.2) reduces the maximum peak to background ratio to a value of $2.6: 1$. Another assumption is that the spectrum of the light is uniform within the cross section of the laser beam. This was confirmed in the measurements we present here. A further reduction of the peak to background ratio would occur when the two beams do not fully overlap in the focus at the SHG crystal, because only the overlapping part will generate the IA signal while the non-overlapping part will add to the background.

Given the proper working of the autocorrelator except for its reduced peak to background ratio, to prepare a judgement of the spectro-temporal properties of the laser output, we present in the following a set of different temporal traces of the temporal intensity of the input light $I(t)$ and the corresponding autocorrelation traces of the autocorrelation intensity $I_{A C}\left(t^{\prime}\right)$. The types of different temporal traces selected in this set is chosen to represent the various expected types of output from the laser, depending on whether it is mode locking or not. Specifically we believe that three different operational modes might be distinguished. The first mode is that of the derived perfect mode locking which generates near-Fourier-limited pulses $\left(\operatorname{sinc}^{2}\right.$ shaped). In this case the spectral spacing of oscillating light frequencies should become perfectly uniform across the entire spectrum. In the temporal domain this corresponds to a sharply defined pulse repetition rate.

Fig. 5.2a illustrates this ideal case. The graph shows the intensity vs. time of the superposition of 49 equidistant light frequencies (spacing chosen as exactly $67 \mathrm{GHz}$ ) with equal amplitudes and equal phases. One sees that the time-trace consists of ultrashort pulses (with a duration of $300 \mathrm{fs}$ ) and that the inter-peak distance is constant (14.9 ps), i.e., a strictly periodic pulse train. Fig. 5.2b shows the autocorrelation trace calculated via Eq.(5.2). It can be seen that for $\operatorname{sinc}^{2}$ shaped pulses the peak to background ratio amounts to $3: 1$ within a resolution error of the calculation of $0.2 \%$. Also, the heights of the correlation side peaks (i.e., for $\left.t^{\prime} \neq 0\right)$ is the same as the height of the center peak $\left(t^{\prime}=0\right)$ due to the strict periodicity of the pulse train, which are the signatures of successful mode locking.

A second considered mode of laser operation is that the frequency comb generated by the laser is still perfectly equidistant, such as in a perfectly dispersion compensated, standard multi-mode laser while, due to a lack of mode locking, the phases of the individual frequencies are randomly distributed. Although such perfectly equidistant formation of a frequency comb is not likely, due to the slightly non-linearity of the grating dispersion in the initial setup, we consider this case as an instructive limiting case for the improved setup. 
Fig. 5.2c shows the intensity vs. time of 49 equidistant light waves with equal amplitude but with random phasing. It can be seen that the resulting time trace shows a multitude of random, smaller spikes. However, due to the strict equidistance of the considered frequency comb, the output is again strictly periodic, with a repetition rate of $67 \mathrm{GHz}$, i.e., each random spike reoccurs with a period of 14.9 ps.

The corresponding autocorrelation trace is given in Fig. 5.2d. It shows that, due to the random phasing, the peak to background ratio is reduced, for our case of 49 waves with equal amplitude, to a value of 1.5:1. It can also be seen that the height of the side peaks is still as high as that of the main peak $\left(t^{\prime}=0\right)$, which expresses the strict temporal periodicity with which the random trace repeats in Fig. 5.2c.

The third considered mode of laser operation is what we believe is most realistic for the initial setup and also possible for the improved setup. The mode of absent mode locking is characterized not only by a random phasing of the light frequencies, but also by a noticeable random deviation of these frequencies from an equidistant pattern. The resulting somewhat randomly spaced spectrum is what we will refer to as quasi-equidistant. Systematic deviations from perfect equidistance is, e.g., typical for standard multi-mode lasers without dispersion compensation. In the initial setup we investigated, the nonlinearity of the grating dispersion is a possible reason for such systematic deviation, as is also expressed in Fig. 4.3. In this setup, however, there are also possible reasons for some additional amount of randomness in the spacing of light frequencies. This is caused by thermally or alignment induced uncertainty in longitudinal mode selection for each of the separate diode lasers comprising the nominally equidistant frequency comb.

Fig. 5.2e displays the intensity vs. time obtained for such an example of 49 superimposed light waves with equal amplitude and random phasing. The 49 light frequencies are chosen only as equidistant on average whereas the individual frequencies deviate randomly from perfect equidistance. In the displayed case, for clarity a strongly random variation of $50 \%$ of the average spacing is chosen. It can be seen that the additional randomness in light frequencies yields random intensity spikes similar to Fig. 5.2c which, however, do not noticeably repeat any more. The randomness in frequency spacing leads to a clear loss of periodicity in the time domain. The corresponding autocorrelation trace as given in Fig. $5.2 \mathrm{f}$ displays this loss of periodicity clearly as well. While the peak to background ratio is unchanged at the center peak $\left(t^{\prime}=0\right)$ at a value of 1.5 , this ratio decreases quickly toward negative and positive delay times.

For completeness, let us notice that the described tendencies do not much change for a smaller number of oscillating modes. With the initial setup, measurements were performed with 26 modes oscillating, in which case a perfect mode locking still yields a maximum $3: 1$ ratio (as in Fig 5.2b), and yields a value of maximum 1.5:1 in Fig. 5.2d and $\mathrm{f}$. 

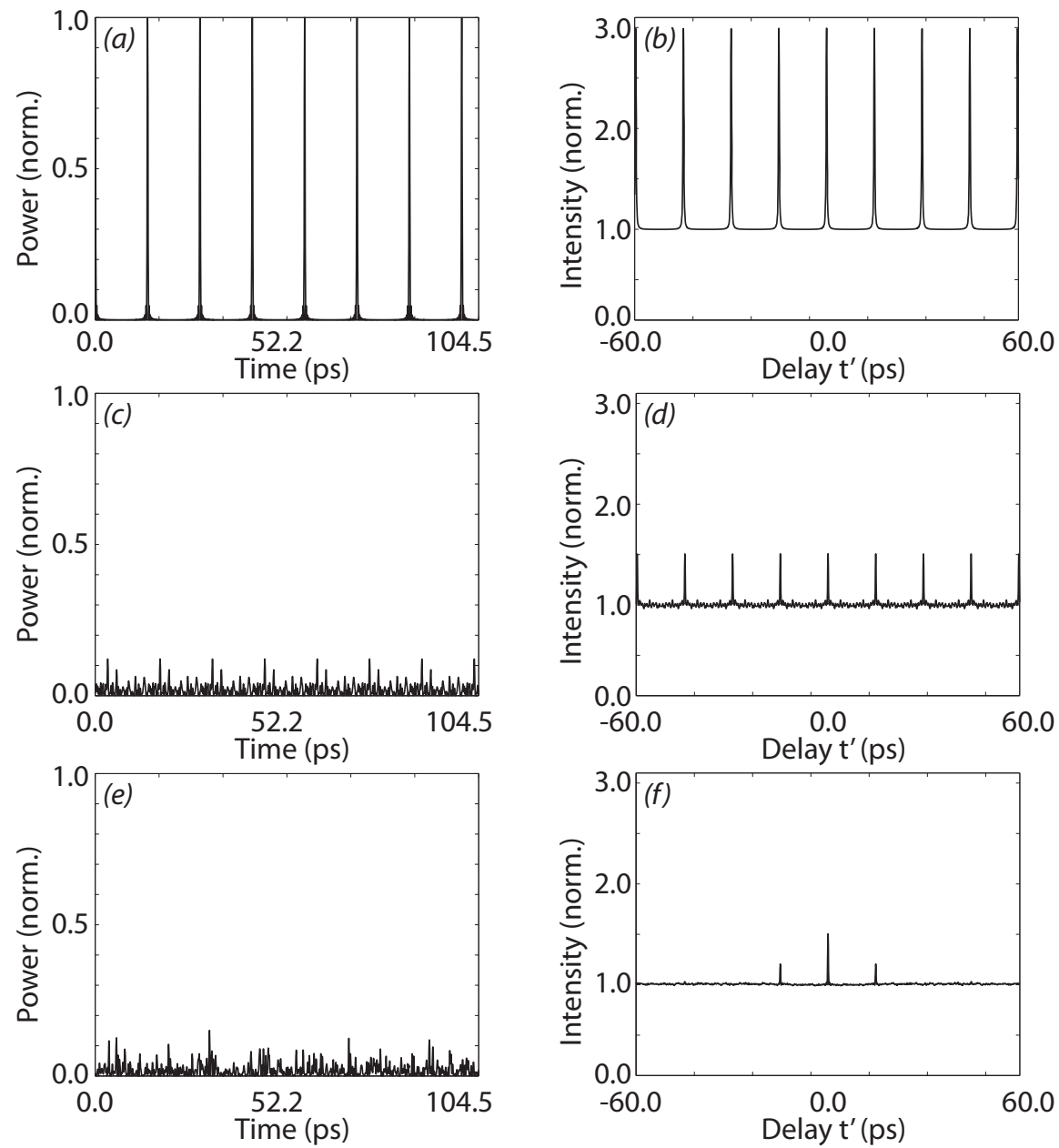

Figure 5.2: (a) the time trace for an equidistant frequency comb, where the individual frequencies have an equal phase. (b) the according calculated intensity autocorrelation (IA) trace, as expected with our type of intensity autocorrelator. (c) a time trace for an equidistant frequency comb, where the individual frequencies have a random phase. (d) the according calculated IA (e) a time trace for a quasi-equidistant frequency comb with a random deviation of $50 \%$ per frequency. $(f)$ The according calculated IA.

In summary, the shown examples of calculated autocorrelation traces can serve as an aid for judging in which mode of oscillation the inspected laser is operating. Peak to background ratios higher than 1.5:1, for instance, would clearly indicate mode locking, a non-decreasing height of the side peaks in the autocorrelation trace would indicate a highly periodic output through equidis- 
tant spacing of light frequencies, no matter whether the laser is mode locked or not.

A peak to background ratio lower than 1.5:1 is however difficult to interpret. The explanation can be the absence of mode locking and also imperfections in the autocorrelator measurements as was mentioned above. The examples shown in Fig 5.2 show clearly that the presence of peaks in the autocorrelation is no certain evidence for the presence of ultrashort pulses, because it can as well be caused by randomly phased modes. Another conclusion is that an autocorrelation trace that shows no peaks at all and only background is most likely caused by a shift of the zero-delay time out of the observation window when inspecting light with a stronger randomness in the spacing of light frequencies.

Certain conclusions can be drawn from the power spectrum, as observed with the OSA, at least as long as spectral changes are involved that are bigger than the instrumental resolution of several GHz. The most prominent example of such a case is if the laser is known to generate a non-equidistant frequency comb where mode locking is certainly absent (when removing the mode locker) and then finding a clearly equidistant spectrum in the same experimental setup (when inserting the mode locker). The transition from a free-running, nonequidistant spectrum to a highly periodic spectrum is a strong indication for a spectral energy transport which requires a temporally periodic modulation of the nonlinear element in the laser, here the SESAM. Although beyond the scope of this Thesis, it should be noted that for certain, unlikely (parabolic) phase spectra for an equidistant frequency comb, the peak to background ratio can be much lower than the mentioned 1.5:1.

\subsection{Initial measurements}

For reference, we first show measurements where a typical spectrum and a typical autocorrelation trace was observed in earlier experiments in Fig. 5.3. The SESAM that was used in these first experiments as concluded from subsequent modeling as in Chapter 4, should not have been able to mode lock the SEGA laser. The reason is that theoretically its specified recovery time was too long $(\tau=4.6 \mathrm{ps})$ and its modulation depth was too small $(\Delta R=5 \%)$, while also its low saturation fluence $\left(\Phi_{\text {sat }}=20 \mu \mathrm{J} / \mathrm{cm}^{2}\right)$ might have been surpassed, which might have driven the SESAM into the range of increased two-photon absorption. Additionally the resonance of that SESAM was specified to lie at a wavelength of $965 \mathrm{~nm}$, which is slightly off the laser's center wavelength of $975 \mathrm{~nm}$. To correct for this deviation we had realigned the grating angle which had led, however, also to a slight change of the expected frequency spacing to a value larger than the originally selected value of $67 \mathrm{GHz}$. Fig. 5.3 shows the first spectral and temporal measurement results obtained with the initial setup of Fig. 5.1. For all other subsequent measurements, a faster SESAM with higher modulation depth as suggested by the modeling (Chapter 4) was used. The 
specifications of this faster SESAM were a modulation depth of $\Delta R=30 \%$, a recovery time of $\tau=500$ fs and a saturation fluence $\Phi_{\text {sat }}=60 \mu \mathrm{J} / \mathrm{cm}^{2}$, around a resonant wavelength of $980 \mathrm{~nm}$.

The measured spectrum is shown in Fig. 5.3a and possesses an average frequency spacing of $71 \mathrm{GHz}$, with 46 emitters in operation. However, as much as $75 \%$ of the spectrum deviates by more than $\pm 3 \mathrm{GHz}$ (which is about the resolution of the OSA) from an equidistant spectrum. The spectrum is equidistant within the OSA resolution only at the 5 lowest frequencies in Fig. 5.3 $(308.8 \mathrm{THz}-309.1 \mathrm{THz})$ and the 6 highest frequencies $(311.7 \mathrm{THz}-312 \mathrm{THz})$, while towards the middle (around $310.3 \mathrm{THz}$ ), the deviation from an equidistant spectrum gradually increases to be as large as $28 \mathrm{GHz}$. The mean deviation is $14 \mathrm{GHz}$ with a standard deviation of $9 \mathrm{GHz}$. All of the recorded spectra with the described setup showed a similarly large deviation from equidistancy, when letting the laser oscillate at the full number of emitters. However, to decrease the deviation, as can also be seen in Fig. 4.3, reducing the number of participating emitters to about 21 emitters in further experiments should restrict the maximum deviation to a value of about $5 \mathrm{GHz}$. The Fig. $5.3 \mathrm{~b}$ shows the typically recorded IA trace for the same setup with 46 emitters in operation. To suppress noise, the presented IA traces are averaged over 128 measurements. It can be seen that the measured signal consists of the measured SHG signal extending over a delay time range of $119 \mathrm{ps}$, in the form of a pedestal. The finite width of the time window is inherent to the construction of the autocorrelator, where the rotation of two glass blocks to realize a time dependent delay, at some point clip the incoming beam. It can be seen that a broad background signal is observed. However, in contrast to the expectations, no peaks are observed in the IA trace. As was discussed before, such observations are likely due to a shift of the point of zero delay in the intensity autocorrelator to lie outside of the 119 ps measurement window. Based on later experiments, we measured that during these first experiments the point of zero delay probably lay $<65$ ps delay toward the negative, which was, however, unknown to us at that time. This shift of delay is consistent with the absence of peaks in the IA trace: already for a maximum frequency deviation from equidistancy bigger than $20 \%$ we calculate that no peaks are observable at delays larger than 45 ps. This seems to suggest that the point of zero delay should be between 45 ps and 65 ps delay toward the negative. 
(a)

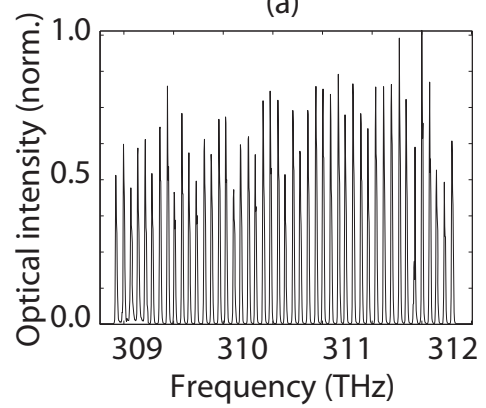

(b)

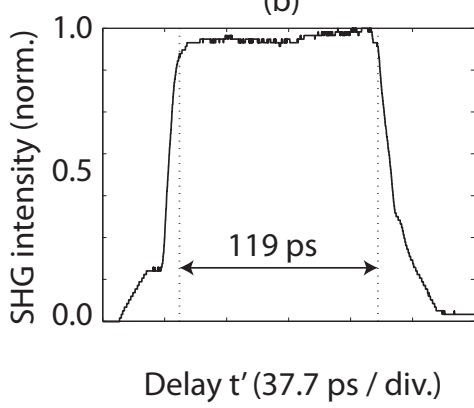

Figure 5.3: (a) a typical frequency comb with a non-equidistant frequency spacing. The largest deviation from an equidistant frequency spacing is $28 \mathrm{GHz}$, while the standard deviation is $9 \mathrm{GHz}$. (b) a typical intensity autocorrelation trace where no peaks are observed. The point of zero delay is likely to be between 45 and 65 ps to the left edge of the pedestal.

To experimentally investigate such a situation of lower systematic deviation from an equidistant mode spacing, we restricted the number of emitters to the central 26 ones, using the adjustable slit in front of the array as described before. The power spectrum and the intensity autocorrelation trace measured with this setting is displayed in Fig. 5.4. The measured maximum deviations (in Fig. 5.4a) from spectral equidistance became indeed lower, thereby reducing also their average values as predicted in Fig. 4.3. The approximate value for the measured systematic deviation from equidistance is also what has been predicted in Chapter 4 and what is displayed in Fig. 4.3. The maximum measured deviation was $7 \mathrm{GHz}$ and the standard deviation was $4 \mathrm{GHz}$, which is close to the approximate $3 \mathrm{GHz}$ resolution of the OSA. The measured average frequency spacing is lowered to a value of $67 \mathrm{GHz}$.

The IA trace as measured with the reduced number of emitters is shown in Fig. 5.4b. To suppress noise, the trace is averaged over 512 measurements. In this measurement, the point of zero delay is still by more than 45 ps and less than 65 ps delay outside of the measurement window. In contrast to Fig. 5.3 there is now a clear set of peaks visible, spaced by a delay time of $14.3 \mathrm{ps}$. The FWHM of the peaks (as measured from the pedestal to the top of the peak) is $2.0 \mathrm{ps}$. The fact that we observe peaks in the autocorrelation, although the point of zero delay is outside of the measurement window can be an indication of mode locking because mode locking, by multiple sideband generation and injection locking induces a spectral equidistance of the participating modes. However, less convincing is that the maximum peak to background ratio of 1.13:1 is much lower than expected for a mode locked pulse train (ideally 3:1). Also the observation that the height of the peaks declines towards higher delay times is not expected for a regular train of pulses. In fact, the decline seems to indicate a randomness in frequency spacing of the oscillating modes. In our 
calculations, we found a similar decline with a random frequency deviation of about $10 \%$. Such deviation from perfect equidistance is also compatible with the direct spectral measurement (Fig. 5.4a), seen the ratio of the resolution of the OSA (about $3 \mathrm{GHz}$ ) and the spacing of frequencies (about $67 \mathrm{GHz}$ ).

(a)

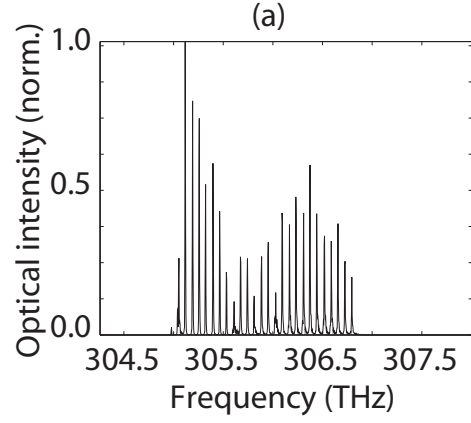

(b)

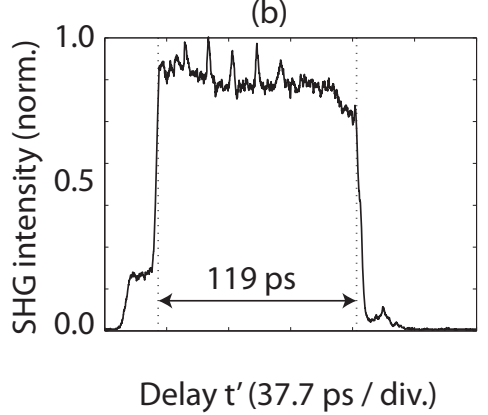

Figure 5.4: (a) measured frequency comb with improved equidistance. The frequency spacing is $66.8 \mathrm{GHz}$. The largest deviation from an equidistant frequency spacing is $7 \mathrm{GHz}$, while the standard deviation is $4 \mathrm{GHz}$. (b) the first intensity autocorrelation trace where peaks were observed (averaged over 512 measurements to suppress noise). The delay between the peaks is $14.3 \mathrm{ps}$. The point of zero delay is likely to be less than 65 ps to the left of the pedestal.

Upon a closer look, the IA trace in Fig. 5.4 (b) contains more indirect spectral information than the directly measured spectrum in the same figure (a). One can see up to five peaks in the IA trace, which span, including the distance to the edge of the recording window, a delay time of about $5 \cdot 14.3 \mathrm{ps}=71.5 \mathrm{ps}$. Since the point of zero delay is less than 65 ps to the left of the lower edge of the recording window, this gives a maximum delay of 136.5 ps over which peaks can still be observed. The width of the individual peaks at FWHM was measured to be smaller than 2 ps. The ratio of the 2 ps and 136.5 ps (approximately $1.5 \%$ ) should roughly correspond to the relative precision with which the temporal trace is periodic. When the peaks in the intensity autocorrelation trace have a maximum deviation of $1.5 \%$, this can only be explained by a correspondingly low spectral deviation from perfect equidistance, namely $1.5 \%$ of the average spacing. In this case, $1.5 \%$ of $67 \mathrm{GHz}$, which is $1 \mathrm{GHz}$. The frequency spacing in the laser, when measured directly with the OSA, cannot be inspected for equidistance with a higher spectral resolution than $3 \mathrm{GHz}$. The spectral spacing that is implied by the temporal measurements (which offers a factor of three better relative precision) yields a very low deviation in spectral spacing $(1 \mathrm{GHz})$, which is not very consistent with the grating diffraction law (see Eq.(4.3) and Fig. 4.3) that should rule the spacing of light frequency without mode locking.

These considerations point to an additional mechanism that was present 
during the measurements of the IA trace and which improves the precision of equidistance in the spectrum. Such a mechanism could point towards mode locking.

To summarize the discussion of these measurements, the results look promising but also inconclusive, whether or not mode locking was achieved. Upon trying to repeat the measurements, we were unfortunately unable to reproduce the results. We believe this was due to heating damage of the SESAM. To achieve saturation fluence we expect to require required $9.2 \mathrm{~W}$ of average power, focused to a spot of approximately an area of $2.3 \cdot 10^{-6} \mathrm{~cm}^{2}$, this gives an optical power density of $4 \mathrm{MW} / \mathrm{cm}^{2}$. The manufacturer does not specify a damage threshold for the average power, but only for pulsed operation. The specified threshold is $300 \mathrm{MW} / \mathrm{cm}^{2}$ for pulsed light with a duty cycle of 1000 . Extrapolating this to a CW threshold which assumes that the specified threshold is thermally induced, this would be comparable to a damage threshold of roughly $300 \mathrm{~kW} / \mathrm{cm}^{2}$ for $\mathrm{CW}$ light. This value is indeed about ten times below the average power that we use, which might explain a permanent damage of the used SESAM.

An experimental option to circumvent thermal damage, is to decrease the average heat load on the SESAM. This can be done in two different ways. First, decreasing the focal spot size at the SESAM, while maintaining the pulse fluence, enables to work with a lower average optical power, thereby decreasing the average heat load on the SESAM. The second method is lowering the repetition rate; if the pulse fluence is maintained, lowering the repetition rate will also reduce the average power, which decrease the average heat load with the same factor. When keeping the focal length of the lens at (d) in Fig. 5.1 constant, to obtain a smaller focus at the SESAM, one could increase the beam diameter in front of (d) which can be achieved with a lens (b) in a larger distance from the array with a correspondingly increased focal length. Such increase in focal length $f_{(b)}$ also inversely proportional decreases the repetition rate. However, this approach will also lead to higher intracavity losses, as was described before, because of the high divergence of the light from the diode bar and the limited physical size of the grating. There would also be an additional alignment sensitivity introduced. Therefore it seems more feasible to decrease the effective pitch of the diode lasers and to reduce the divergence of the light in front of lens (b), as will be discussed in Section 5.5.

Finally, the inherent, slight parabolic non-equidistance of the light frequencies (as seen in Fig 4.3) would be left unchanged, if one changed only the overall focusing conditions and average repetition rate. Seen these disadvantages we decided to follow a rather different approach which is described in the following as the improved setup. 


\subsection{Improved setup}

The discussed issues for non-equidistant frequency comb and possibly heating induced damage of the SESAM formed the motivation to devise the improved setup as shown in Fig. 5.5. The issues are addressed by implementing an optical waveguide chip (pitch converter) in the cavity. The pitch converter is fabricated using $\mathrm{Si}_{3} \mathrm{~N}_{4} / \mathrm{SiO}_{2}$ waveguide technology (TriPleX ${ }^{T M}$ ) [22].

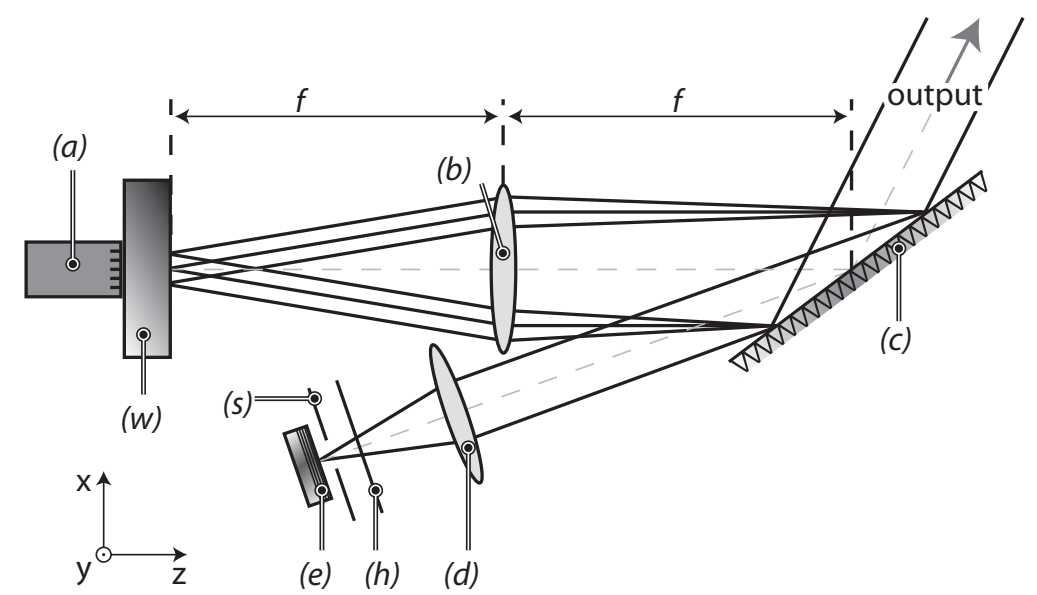

Figure 5.5: Overview of the experimental setup of the SEGA mode locked laser. Light originates from the AR coated diode bar (a). An optical waveguide chip $(\mathrm{w})$ is butt-coupled to the diode bar. Light emitted from the waveguide chip is collimated by a spherical lens (b). The light is combined by a diffraction grating (c), and focused by a lens (d) on the SESAM (e). The collimation lens (b) is placed one focal length $f_{(b)}$ from the diode bar, and the diffraction grating (c) is one focal length $f_{(b)}$ from the lens (b). The output of the laser can be changed by rotating a $\lambda / 4$ plate $(\mathrm{h})$. An adjustable mechanical slit (s) is placed in front of the SESAM.

The design of the pitch converter, with an array of waveguides, aims on providing an equidistant frequency comb in our laser which requires, according to Eq.(4.3), a suitably non-equidistant spacing (pitch) of emitters. Secondly, the design aims on achieving a smaller focus of the beam at the SESAM, to enable operation with a lower average power and thus a lower heat load.

Fig. 5.6 shows top view microscope images (recorded in the y-direction of the setup) of the two pitch converters that were investigated. The images were taken after carefully aligning the left hand side facet of the chip with the output facet of the diode bar, with the diodes turned on. The images then show the spatial distribution (in the x-z-plane) of the residual, scattered light, while the main portion of the light flows through the waveguide array to the right hand side output facet of the chip. The images thereby display the location and 
spacing (pitch) of the individual waveguides. It can be seen that seven out of the 49 waveguides do not show up as scattered light. The reason is that in the used diode bar the corresponding diode laser elements had become inoperative.

The considerations for the chosen design parameters are as follows. On the input side (Fig. 5.6a) the spacing of the waveguides is chosen as equidistant to optimally match the positions of the diodes in the diode bar (constant pitch of $200 \mu \mathrm{m})$. Near the input side, the width of the waveguides in the $\mathrm{x}$-direction is varied (tapered) along the $\mathrm{z}$-direction. The taper is chosen to match the beam radius of the input facet of the waveguides to that of the output light from the diode facets. This maximizes the input coupling from the diodes into the pitch converter. To reduce Fresnel reflections, the facets of the waveguide chip are polished and anti-reflection coated. Within the waveguide chip, i.e., between the input and output facets, the waveguides were chosen to have a rectangular cross section of the higher index $\mathrm{Si}_{3} \mathrm{~N}_{4}$ core, with a width of $800 \mathrm{~nm}$ and a thickness of $30 \mathrm{~nm}$. This choice of cross section was motivated by fabrication compatibility with the tapers and the low propagation losses that can be achieved $(<0.1 \mathrm{~dB} / \mathrm{m}[72])$.

On the output side the waveguides are tapered as well, to achieve an approximately round beam diameter at the facets with a minimum astigmatism, together with a limited divergence. To more detail, the taper is designed to collimate the full beam with the lens (b). For the design value of the taper we calculate an output beam diameter at the facets of $11.0 \mu \mathrm{m}$ in the $\mathrm{x}$-direction and $10.9 \mu \mathrm{m}$ in the y-direction. Divergence causes the calculated beam diameter to be about $3.5 \mathrm{~cm}$ in a distance of $30 \mathrm{~cm}$, i.e., at the position of lens (b). This beam diameter is deliberately chosen to be somewhat smaller than the $5 \mathrm{~cm}$ diameter of the lens to account for fabrication errors in waveguide thickness. To give an example for the influence of the named errors, the beam diameter at the facet yields the named divergence with a waveguide thickness of $30 \mathrm{~nm}$ and a waveguide width of $800 \mathrm{~nm}$. However, an increase of the waveguide thickness by just $5 \mathrm{~nm}$ with equal waveguide width decreases the beam diameter at the facets to $6.8 \mu \mathrm{m} \times 6.5 \mu \mathrm{m}$, which increases the divergence by roughly a factor of 1.6 and the beam size to $5.5 \mathrm{~cm}$ at the position of lens (b). Additional deviations from the designed beam diameter at the facet may occur because the waveguide is polished after fabrication. This can easily cause a decrease in taper length of several tens of micrometers, on a total taper length of $500 \mu \mathrm{m}$. Indeed, a measurement of the beam diameter at lens (b) yielded a value close to $5.5 \mathrm{~cm}$, which confirms the presence of small fabrication errors as described.

For the experiments described here, we designed two different pitch converters, one for achieving an equal frequency spacing of $67 \mathrm{GHz}$ and one for an equal frequency spacing of $20 \mathrm{GHz}$.

The required, slightly non-uniform pitch at the output side (around $200 \mu \mathrm{m}$ for $67 \mathrm{GHz}$ and around $57 \mu \mathrm{m}$ for $20 \mathrm{GHz}$ ) was calculated as follows. We numerically solved Eq.(4.3) for the emitter spacing, $d$, as function of emitter 
number $n$, for the derived equidistant spacing of the frequencies, $\nu_{n}$, and the given grating period, $\Lambda_{g}$ and angles $\theta_{i}$ and $\theta_{0}$.

The remaining parts of the setup remained basically unchanged. The beam size behind the grating had a measured cross section of $5 \mathrm{~cm}$ (vertically) and $1.2 \mathrm{~cm}$ (horizontally). The light is focused on the SESAM by lens (d), with a focal length of $f_{(d)}=200 \mathrm{~mm}$. The calculated focal spot area at the SESAM is $8.2 \cdot 10^{-7} \mathrm{~cm}^{2}$, which is a factor of 2.8 smaller than that in the initial setup. This also indicates that the average power, i.e., the heat load, on the SESAM can be 2.8 times lower, while still achieving the saturation fluence.

The heat load can be lowered even further if using the pitch converter with the smaller pitch such that the repetition rate is lowered from $67 \mathrm{GHz}$ to $20 \mathrm{GHz}$. For the experiments described here, we designed two different pitch converters: one which results in an equal frequency spacing of $67 \mathrm{GHz}$ and one which results in an equal frequency spacing of $20 \mathrm{GHz}$. According to the following estimate, in order to reach the SESAM's saturation fluence of $\Phi_{\text {sat }}=60 \mu \mathrm{J} / \mathrm{cm}^{2}$ with pulses of $20 \mathrm{GHz}$ repetition rate, an average power of $1.0 \mathrm{~W}$ is required. With the other pitch converter $(67 \mathrm{GHz})$ an approximately three times higher power of $3.3 \mathrm{~W}$ would be required.

(a)

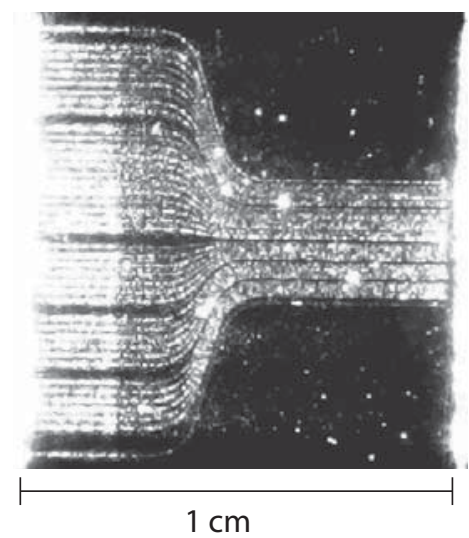

(b)

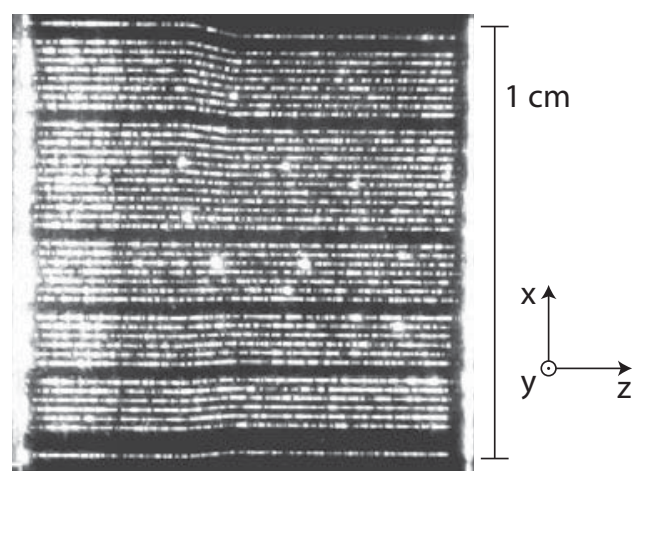

Figure 5.6: A microscope picture (top view) of the used pitch converters when light from the diode array (on the left hand side, not shown) is coupled in. The bright lines indicate, via residual scattering of light out of the waveguides, where the waveguides are located in the $x$-z plane. (a) shows the $20 \mathrm{GHz}$ pitch converter, (b) shows the $67 \mathrm{GHz}$ pitch converter. The missing lines are not due to missing waveguides, but due to defective diode lasers. 


\subsection{Improved setup measurements and results}

For the first experiment with the improved setup, we used the $67 \mathrm{GHz}$ pitch converter. Based on extrapolation of power measurements, we expect to achieve the average power required for a saturation of the SESAM $\left(\Phi_{\text {sat }}-60 \mu \mathrm{J} / \mathrm{cm}^{2}\right.$ in presumed pulsed operation) at a pump current of $35 \pm 5$ A. However, to stay safely below damage threshold of the waveguides, we chose to increase the pump current only until we observed peaks in the IA trace. This was first clearly observed at a pump current of $19 \mathrm{~A}$, while no pulses were observed below this value.

With this setup, we observed an equidistant frequency spacing (Fig. 5.7) with an average frequency spacing of $69.6 \mathrm{GHz}$. This value is close to what is expected from the pitch of waveguides at the output facet according to Eq.(4.3). In this quantification we assumed that missing frequencies, due to inoperable diode lasers do not contribute to frequency deviations. To more detail, different from the initial setup, we did not find any systematic spectral variation of the frequency deviations from their average, only a seemingly random variation was present. Within $60 \%$ of the spectrum (34 frequencies, from 306.795$309.089 \mathrm{THz}$ ) we find a standard deviation of $5 \mathrm{Ghz}$ and a single maximum deviation of $16 \mathrm{GHz}$. This is measured again with a resolution of several $\mathrm{GHz}$, as provided by the OSA. The next largest maximum deviation is $7 \mathrm{GHz}$, which is $\sim 10 \%$ of the frequency spacing.

The IA trace as measured with this setup is shown in Fig. 5.7b. For this measurement, the point of zero delay was realigned to be positioned at the center of the measurement window. A clear set of peaks is visible, with a delay time of $14.4 \pm 2 \mathrm{ps}$ between the individual peaks. The FWHM of the peaks as measured from the pedestal to the top of the peak is about $2 \mathrm{ps}$. The peak to background ratio is 1.04:1 for zero delay, declining towards higher delay times. The low peak to background value is much smaller than expected for a regular train of pulses and therefore it seems to indicate a random phasing of the comb frequencies. The observed decline in peak to background value for delay times smaller and bigger than zero is consistent with a $10 \%$ random frequency deviation from a fully equidistant spacing. This value is in agreement with the spectral measurement. 
(a)

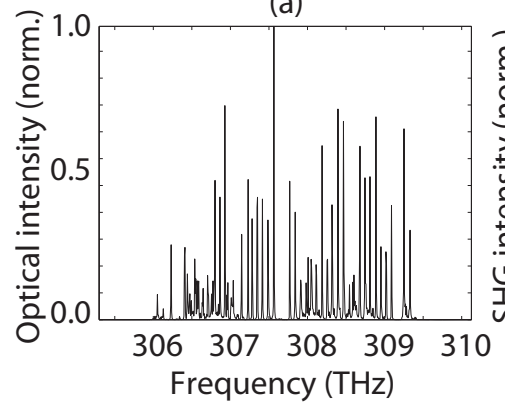

(b)

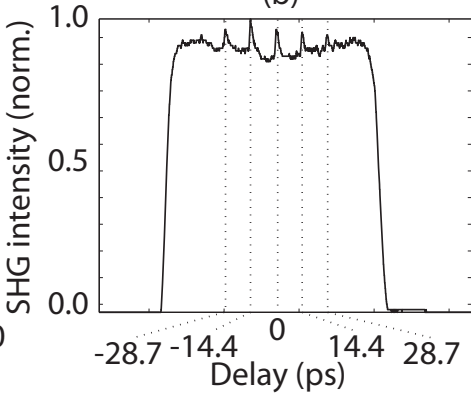

Figure 5.7: (a) a measured frequency comb with an approximately equal frequency spacing from 306.795-309.089 THz. In this frequency range, the frequency spacing is $69.6 \mathrm{GHz}$, the largest deviation from an equidistant frequency spacing is $16 \mathrm{GHz}$ for a single frequency, the next largest maximum deviation is $7 \mathrm{GHz}$, and the standard deviation is $5 \mathrm{GHz}$. (b) the according intensity autocorrelation trace where peaks were observed. The delay between the peaks is 14.4 ps. The measurements are performed with the $67 \mathrm{GHz}$ pitch converter and a SESAM in the setup.

The interpretation of these data, when comparing to the various possibilities presented in Fig. 5.2, indicates that a mode locking was not achieved with these settings. The main hints for this are a rather small peak to background ratio and a relatively strong decline of IA peak vs. delay. A possible explanation for the absence of mode locking is that the average power of the laser is lower, about $2 \mathrm{~W}$, than the presumably required $3.3 \mathrm{~W}$ to reach saturation fluence in pulsed operation.

For the second experiment, we used the $20 \mathrm{GHz}$ pitch converter. The presumably required average power of about $1 \mathrm{~W}$ (to reach $\Phi_{\text {sat }}$ ) is achieved at a pump current of $11 \pm 1 \mathrm{~A}$. In contrast to the previous experiment, to provide an even stronger saturation for pulsed operation we chose to use an increased pump current of $20 \mathrm{~A}$.

With this setup we observed an average frequency spacing of $20.8 \mathrm{GHz}$ (see Fig. 5.8). In terms of absolute numbers the spectrum is rather equidistant, with $67 \%$ of the spectrum (30 frequencies from 307.51-308.12 THz) showing a very small standard deviation of $2 \mathrm{GHz}$, and with a maximum deviation of $5 \mathrm{GHz}$. In terms of relative numbers, however, these values correspond to a $10 \%$ standard deviation and a maximum of $20 \%$ deviation from the average of $20.8 \mathrm{GHz}$.

The IA trace as measured with this setup is also shown in Fig. 5.8. A clear set of peaks is visible, with a delay time of $48 \pm 2$ ps between the individual peaks. The FWHM of the peaks (as measured from the pedestal to the top of the peak) is 2 ps. The peak to background ratio is 1.11:1 at zero delay, declining towards higher delay times. The zero delay value is again much lower 
than expected for a regular train of pulses, indicating a random phasing in the frequency comb. The strength of decline in peak to background value vs. delay is consistent with a slightly randomized spacing of the involved output frequencies. Our calculations show a similar decrease in IA peak intensity with a frequency deviation of about $20 \%$. This frequency deviation is in agreement with the spectral measurement.

(a)

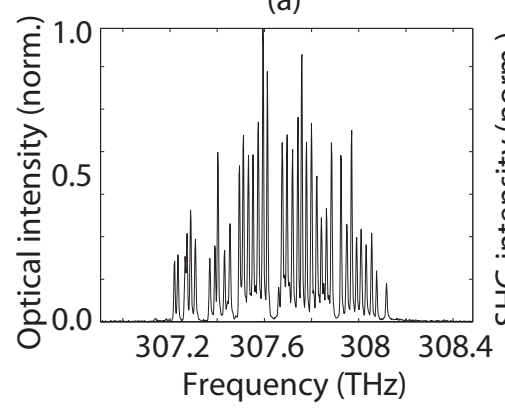

(b)

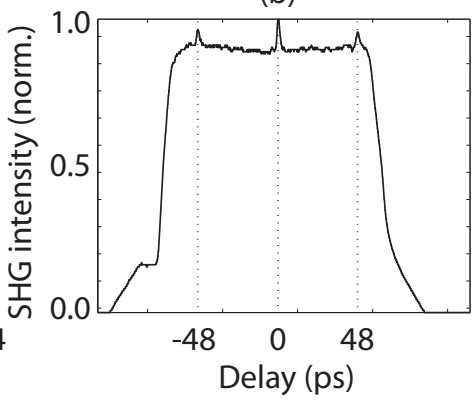

Figure 5.8: (a) a measured frequency comb with an approximately equal frequency spacing from 307.51-308.12 THz. In this frequency range, the average frequency spacing is $20.8 \mathrm{GHz}$, the largest deviation from an equidistant frequency spacing is $5 \mathrm{GHz}$, while the standard deviation is $2 \mathrm{GHz}$. (b) the according intensity autocorrelation trace where peaks were observed. The delay between the peaks is $48 \mathrm{ps}$. The measurements are performed with the $20 \mathrm{GHz}$ pitch converter and a SESAM in the setup.

In summary, the presented results do not show any clear signs of mode locking. There seems to be a better consistency with random phased light frequencies that are also slightly randomly spaced.

There is, finally, an observation that we made systematically throughout all of the described experiments. Whenever a SESAM was used instead of a plain mirror, the output spectrum became quite stable in the following sense. The variation of power from the individual emitters (i.e., the height distribution of peaks in the output spectrum) became noticeably smaller, while the spacing of emitted light frequencies became noticeably more equidistant. This can be seen, for example, by comparing the spectrum in Fig. 5.8 (recorded with a SESAM in the resonator) with the spectrum in Fig. 5.9 (recorded with a plain mirror, E03, Thorlabs). In order to see whether the difference in output also shows up in a temporal measurement, we performed an autocorrelation measurement with the plain mirror. The results are displayed in Fig. 5.9. Next to the spectrum with poorly equidistant frequencies and strong varying spectral power, Fig. 5.9b displays the recorded IA trace. The trace shows three peaks separated by a delay time of $48 \pm 2 \mathrm{ps}$. The central peak is higher than the background with a ratio of of $1.18: 1$. The decline of the side peaks is consistent with a spectral 
frequency deviation of $>60 \%$. Due to the absence of a mode locker or any other apparent nonlinear optical response via which modes of different diode lasers can control their phases, these modes need to be randomly phased. The strong decline of the side peaks proves a strong randomness in the spectral spacing of modes as well. Interestingly, the height of the side peaks, compared to the central peak is smaller than when the SESAM is present, roughly a ratio of 1:2 vs. 1:3. This seems to indicate that placing the SESAM in the cavity increases somewhat the temporal periodicity of the laser output. This is essentially the same statement as was made before, that the SESAM generates a larger extent of spectral order. Such phenomena can, however, only be understood if the SESAM is indeed operating in its saturated regime where the mode beating of incident light gives rise to spectro-temporal changes in the reflected light.

(a)

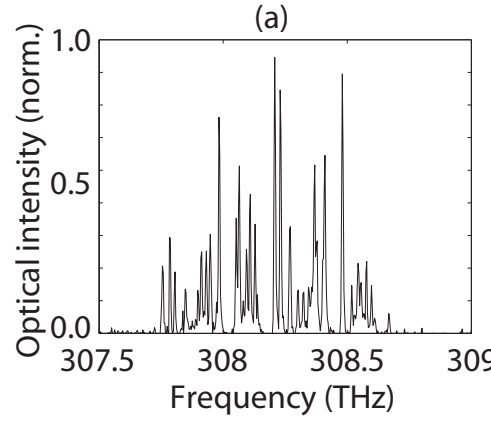

(b)

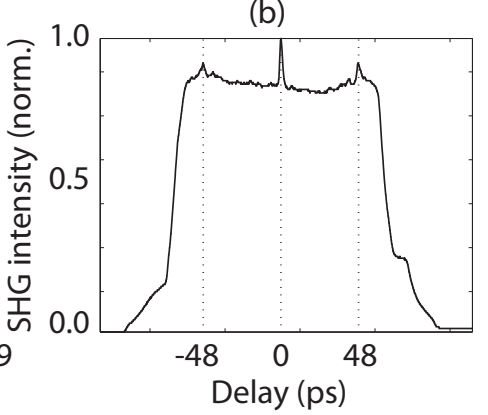

Figure 5.9: (a) a measured spectrum where no equidistant frequencies can be determined. This spectrum is so poorly equidistant that we could not properly statistically analyze it. (b) the according intensity autocorrelation trace where peaks were observed. The delay between the peaks is $48 \mathrm{ps}$. The measurements are performed with the $20 \mathrm{GHz}$ pitch converter and a mirror in the setup.

To verify whether or not the SESAM is driven into saturation with the named operation parameters, a final measurement was performed. The measurement consists of simply recording the output power from the laser as a function of pump current. For this measurement, the $20 \mathrm{GHz}$ setup is used with either the SESAM or the plain mirror installed. The expectation is that, if the SESAM reaches saturation, its reflection will increase. The output power should then also increase yielding a stronger than the initial slope of growth vs. pump current. For the laser above threshold, but with a plain mirror installed, the output power as function of pump current should exhibit only a linear increase. The experimental results are shown in Fig. 5.10. It shows that for the laser with the plain mirror, a linear slope of output power vs. pump current is measured, as expected.

In contrast, for the laser equipped with the SESAM the output power first attains a linear increase of power (between 8 and $15 \mathrm{~A}$ of pump current). At higher current, between 16 and $23 \mathrm{~A}$, the slope of growth is clearly steeper than 
before, finally, the slope is reduced to close to the initial value (23 to $28 \mathrm{~A}$ ). This nonlinear increase beginning at 15 A pump current seems to indicate that the SESAM's saturation fluence is reached. This observation can be compared to the specifications of the SESAM. The average power of the laser that reaches the SESAM at this pump current is about $1.5 \mathrm{~W}$. This is somewhat higher but still well comparable with the $1.0 \mathrm{~W}$ we calculated before to reach saturation fluence. The difference might be caused by deviations from the Gaussian beam profile of the laser beam that is focused onto the SESAM, which will increase the focal spot size and therefore lowers the fluence. However, the named saturation parameter, which is a fluence, is actually only of relevance for pulsed operation. Our calculations (using the calculation code of Chapter 4) show that for 33 emitters, the difference in output power is about $5 \%$ higher at saturation fluence if the laser is in pulsed operation, compared to light with a quasi-equidistant light spectrum with random phasing. At an average power corresponding to the SESAMs saturation fluence (we calculated this to be $1 \mathrm{~W}$ for our case), the increase in output power when an average power for the random light fluctuations, due to the quasi-equidistant spectrum is marginal (only 0.7\%). For pulsed operation the increase in output power would be $6 \%$. At higher fluences, the difference increases even further. As example, for an average power corresponding to twice the saturation fluence, the laser in pulsed operation would already have an average output power of $10 \%$ higher compared to the laser with the quasi-equidistant light spectrum and random phasing. It is therefore very unlikely that the temporal nature of the light flow which caused the nonlinear increase in laser output power in Fig. 5.10, and thus obviously saturated the SESAM, is that of a continuous wave.

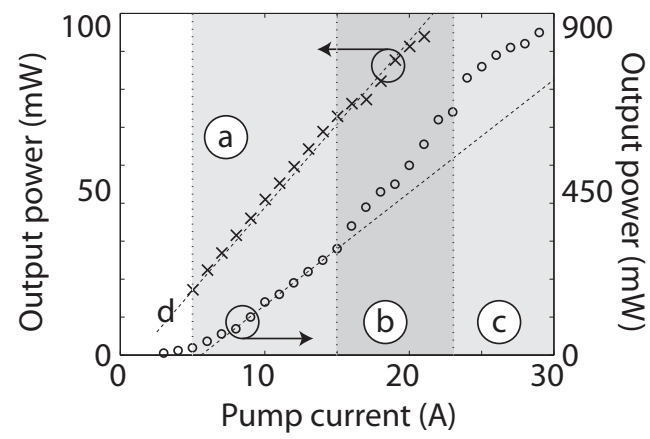

Figure 5.10: Output power of the SEGA laser as a function of pump current. (-o-) denotes the measurements performed with a SESAM in the cavity. The dotted line shows a linear dependency above threshold, in the gray shaded area (a). Beyond 15 A pump current a growth of output sets in with a higher slope (gray shaded area (b)), followed by growth with a reduced slope (gray shaded area (c)). (-x-) denotes measurements performed with a mirror in the cavity. The growth can be roughly described with a linear growth following a single slope (dotted line (d)) until the growth diminishes. 


\subsection{Conclusions}

In this chapter we have presented measurements for separate gain (SEGA) mode locking. Measurements on the first setup showed a single occasion where an equidistant frequency comb with a frequency spacing of $66.8 \mathrm{GHz}$ was observed. This occasion also showed peaks in the intensity autocorrelation (IA) trace for the first time. However, the results suggested that the remaining nonlinearity in the dispersion of the feedback grating formed a problem to obtain a sufficiently high equidistance of the generated frequency comb. Additionally, results could not be improved or reproduced, probably due to thermal damage of the used SESAM through operation with a reduced number of diode elements at an increased power. To solve these difficulties we improved our setup based on our calculations of Chapter 4. The modifications consisted in the design, fabrication and introduction of different pitch converters which effectively modifies the pitch of the diode lasers in the diode bar and also offers, via tapered output ends of the waveguides in the pitch converter, a better focusability for operation at low average power. Equidistant frequency combs were achieved with two different pitch converters. With a $67 \mathrm{GHz}$ pitch converter, a $69.6 \mathrm{GHz}$ frequency spacing was achieved, equidistant within $5 \mathrm{Ghz}$ for $60 \%$ of the spectrum, which is close to the resolution limit of the used optical spectrum analyzer. With a $20 \mathrm{GHz}$ pitch converter a frequency spacing of $20.8 \mathrm{GHz}$ was achieved, equidistant within $2 \mathrm{Ghz}$ for $67 \%$ of the spectrum. The spectral measurements generally show approximately equidistant frequency combs in which the degree of equidistance increases when a SESAM is used in the cavity compared to what is observed with a plain mirror. To inspect the temporal properties of the output with ultrafast time resolution, we measured the intensity autocorrelation with a setup that is based on collinearly phase matched second harmonic generation. The intensity autocorrelation traces (second harmonic power vs. delay) show peaks at a spacing that corresponds to the expected repetition rate. However in all cases the peak to background ratio was much smaller than expected for a Fourier limited pulse train and, also, for a random superposition of the modes oscillating in the frequency comb. Calculations show that the IA measurements can be explained by an almost equidistant frequency comb with random phases, taking into account the measured power imbalance in the two arms of the autocorrelator and a somewhat incomplete beam overlap. When a mirror rather than a SESAM is used, similar IA traces are obtained with similar peak to background ratios at zero delay. However, when a mirror is installed in the laser, the peaks at non-zero delay decrease more rapidly than with a SESAM. The corresponding improvement of equidistance in the frequency combs can also be seen directly in the spectral measurements. As the spectral equidistance of the individual frequencies in the frequency comb became increased by feedback from the SESAM, the measurements imply that the SESAM exerts some of the intended control which is an equidistant spectrum through mode locking to generate a pulsed output.

Further evidence that feedback from the SESAM contributes to nonlinear 
effects in the dynamics of the laser comes from measurements of the average output power. The output power is found to increase nonlinearly with increasing pump current from 15 A upward, when the SESAM is present in the cavity. This is in contrast to what is observed when a plain mirror is used for feedback, where the output power increased linearly.

Although the currently available results are inconclusive, they also bear much insight and promise for a demonstration of mode locking in subsequent experiments. It might be essential to replace the diode bar, such that all emitters are operational again. This will provide better exchange of energy and phase locking across the entire spectrum, providing the potentially shortest pulses due to an improved saturation of the saturable absorber with minimum average power. Currently, these mechanisms seem to be restricted only to groups of modes in which none of the comb frequencies is missing. A second improvement in the experimental setup would be to acquire a diode bar with a slightly nonlinear pitch, such that the current combination of a constantpitch diode bar with a variable-pitch waveguide converter can be replaced by one single device. This will have the advantage that the setup becomes more stable, because the waveguides do not need to be carefully aligned anymore.

Also on the measurement part of the experiments improvements can be made. First, the used autocorrelator proved difficult to work with because the device is designed for standard, actively mode locked solid state lasers which operate in a different wavelength range with much higher pulse energy via orders of magnitudes lower repetition rate. It would be important to devise an intensity autocorrelator specifically designed for the combination of lower duty cycle and peak power due to the extremely high repetition rates, in the form of a Michelson interferometer offering a larger range of delay times. Our calculations show, that equidistance in the spectrum can be observed best, i.e., with highest resolution, via the decline in height of autocorrelation peaks towards larger delay, compared to direct measurements with the optical spectrum analyzer. Secondly, if the SEGA laser is expected to be mode locking, FROG measurements can be performed to confirm pulse length, spectral equidistance and phase relation. Alternatively, a streak camera could be used to determine the pulse length.

From our measurements and calculations we conclude that we are on the verge of an experimental demonstration of SEGA mode locking. By implementing the suggested improvements, one could make an important step towards a first demonstration and stable mode locking of separate gain media. This would be advantageous for possible applications of mode locked lasers where a high repetition rate and high average output power is required. An example is driving optically nonlinear processes towards highest repetition rates, such as ultrafast, all-optical switching $[9,10]$ which is relevant for all-optical computing with clock frequencies in the $\mathrm{THz}$ range. 



\section{6 \\ Conclusions}

In this thesis we have investigated an innovative approach for the spectral control of diode lasers that uses integrated optics waveguide circuits for an optical feedback in the form of an external cavity. In the first two chapters, we have focused on the theory and experiments of such waveguide based external cavity semiconductor laser (WECSL). Chapters three and four are on the theory of and experiments on mode locking a laser with spatially separate gain (SEGA) media, referred to as SEGA mode locking.

The most important conclusion to draw from the theoretical work on WECSLs is that the concept to replace standard external cavities based on bulk optics for diode laser control with low loss waveguide circuits involving waveguide resonators is highly promising. Specifically, the concept is capable to provide small-sized external cavity diode lasers emitting a single frequency, providing wide tuneability and importantly a very narrow bandwidth. In this Thesis we started to explore this concept with the specific example of feedback with a highly frequency selective waveguide circuit as expected from two slightly differently sized micro ring resonators (MRRs) in series. The designed circuit is what we call an MRR mirror. The waveguide circuits were available as fabricated with low loss $\mathrm{Si}_{3} \mathrm{~N}_{4} / \mathrm{SiO}_{2}$ technology and the design of the circuits was optimized to obtain single frequency oscillation over a wide spectral range, in this case across the telecommunication C-band (around $1550 \mathrm{~nm}$ ).

Indeed, in the experiments we have measured a wide tuning range of over $42.3 \mathrm{~nm}$, covering the more than entire C-band. The WECSL had a measured narrow bandwidth of $25 \mathrm{kHz}$, which we believe is currently only limited by our particular choice of design parameters of the waveguide circuits. This conclusion is based on our theoretical estimate of the spectral bandwidth which shows reasonable agreement with the experiment. Looking at the parameters that determine the bandwidth shows that the bandwidth might be lowered by orders of 
magnitude by applying several improvements that reduce the total losses of the external feedback. A first measure would be designing an improved coupling between diode laser and waveguide by waveguide tapering, which matches the mode overlap of the two components and thereby reduces the coupling losses to only scattering and misalignment losses. A next improvement would be the removal of an additional output coupler that has so far been used for monitoring the cavity-internal light flow. This gives a straightforward reduction of losses in the feedback (around 11\%). Another improvement is expected by decreasing the losses associated with the double ring circuit, which are occuring as scattering at couplers, as propagation losses and as bending losses. For instance, it can be considered to replace the box-shaped waveguide cross section by a single, rectangular one, as the latter had shown upto 60 times lower propagation loss $[33,72]$.

A second way to reduce the spectral bandwidth of the laser is by optimizing the feedback parameters of the micro ring resonators, to increase the finesse of the MRR mirror and thereby also the finesse of the entire WECSL resonator. A measurement of the effective finesse of the complex resonator structure of WECSL after assembly, i.e., including the effects of residual losses at facets, can be done via injection locking. For instance, we have determined the effective finesse of our WECSL resonator by measuring the locking range. Monitored by such measurements, the finesse can be increased by reducing the feedback bandwidth. This can be done by increasing the finesse of the MRRs themselves, by decreasing the coupling coefficient, $\kappa$, and thereby decreasing the bandwidth of the reflection. However, decreasing the coupling coefficient causes resonant light to travel many more roundtrips through the MRRs before being reflected back to the laser. This increases the overall loss, due to the radiative bending losses and scattering losses inside the MRRs. In summary, the lowest reflected bandwidth for the given waveguide geometry can be achieved by optimizing for the highest finesse by lowest coupling coefficient, while maintaining sufficient feedback for the laser. Other geometries might be worth investigating, such as based on two identical resonators, not in series but mutually coupled, to reduce the feedback bandwidth $[40,41]$.

The narrow bandwidth and great potential for significant further narrowing, fast wavelength switching, wide wavelength tuneability and the demonstration of injection locking makes WECSLs a promising technological approach for advanced applications such as in broadband phased array antenna systems.

In the second part of this Thesis, we have introduced an interesting innovative method of mode locking called separate gain mode (SEGA) mode locking. Our approach promises to lift certain limitations on the combination of repetition rate and output power. It also enables a decoupling of the pulse repetition rate from the need to realize a matching cavity length. Modeling of this laser shows with which sets of laser parameters one might be able to demonstrate such mode locking for the first time. Specifically, what the calculations reveal are the physical properties that are required for the nonlinear feedback element, in our considerations a semiconductor saturable absorber mirror (SESAM). We 
have used our model to analyze mode locking of a specific laser, which is a diode laser bar with $49-\mathrm{W}$ average output power, $67-\mathrm{GHz}$ repetition rate and 300fs pulse duration. The ongoing development of faster SESAMs with a higher modulation depth [14] makes it realistic to assume that mode locking over a wide range of average powers and repetition rates is achievable. Also, the model can also be applied to investigate SEGA mode locking requirements for other types of laser or at different combinations of output power, repetition rate and pulse duration.

For experimental investigation of the SEGA laser we have measured both the spectral and temporal behavior of such a laser. We have devised two different types of setups, one for a repetition rate of $67 \mathrm{GHz}$ and one for a repetition rate of $20 \mathrm{GHz}$. We have observed behavior that can be explained by a frequency comb with random phases and a small frequency deviation per emitter. These observations include an almost (but not exactly) equidistant spectral frequency comb and peaks in the intensity autocorrelation trace, which are not consistent with that for a mode locked laser. However, we have also observed behavior that can better be explained by a regular train of mode locked pulses. The main observations include an increased spectral equidistance when a SESAM is employed as feedback mirror, compared to a plain mirror. Further evidence that the feedback from the SESAM contributes to nonlinear effects in the dynamics of the laser comes from a measurement of the average output power. The output power is found to increase nonlinearly with increasing pump current, when the SESAM is present in the cavity. This in contrast to what is observed when a plain mirror is used for feedback, where the output power increased linearly.

Although the currently available results are inconclusive, they also bear much insight and promise for a demonstration of mode locking in subsequent experiments. It might be essential to replace the diode bar, such that all emitters are operational again. This will provide better exchange of energy and phase locking across the entire spectrum, providing the potentially shortest pulses due to an improved saturation of the saturable absorber with minimum average power. Currently, these mechanisms seem to be restricted only to groups of modes in which none of the comb frequencies is missing. A second improvement in the experimental setup would be to acquire a diode bar with a slightly nonlinear pitch, such that the current combination of a constantpitch diode bar with a variable-pitch waveguide converter can be replaced by one single device. This will have the advantage that the setup becomes more stable, because the waveguides do not need to be carefully aligned anymore. Also on the measurement part of the experiments improvements can be made. It would be important to devise a background-free intensity autocorrelator specifically designed for the combination of lower peak power but extremely high repetition rates, in the form of a Michelson interferometer offering a larger range of delay times. Our calculations show, that equidistance in the spectrum can be observed best, i.e., with highest resolution, via the decline in height of autocorrelation peaks towards larger delay, compared to direct measurements 
with the optical spectrum analyzer. Secondly, if the SEGA laser is expected to be mode locking, FROG measurements can be performed to confirm pulse length, spectral equidistance and to retrieve phase spectra. Alternatively, a streak camera could be used to determine the pulse length.

From our measurements and calculations we conclude that we are on the verge of an experimental demonstration of SEGA mode locking. By implementing the suggested improvements, one could make in important step towards actual stable mode locking of separate gain media. This would be advantageous for possible applications of mode locked lasers where a high repetition rate and high average output power is required. As examples, we recall ultrafast, all-optical switching $[9,10]$ which is relevant for all-optical computing with clock frequencies in the $\mathrm{THz}$ range and photomagnetic switching [8] with Tbit/s reading and writing speed. An interesting notion might be to use multiple WECLSs in an integrated optical waveguide chip setting, to achieve SEGA mode locking on chip. This might have use in the named applications of integrated optical computing, since the SEGA mode locking setup in this Thesis is proposed in free space, and is therefore still rather bulky. 


\section{A}

\section{Appendix A}

In the SEGA mode locking model, Chapter 4, Section 4.4.2, the gain per emitter $\left(g\left(I_{t o t}\right)\right)$, including gain saturation, is given as

$$
g\left(I_{\text {tot }}\right)=\frac{g_{0}}{1+I_{\text {tot }} / I_{\text {sat }}},
$$

where $I_{t o t}$ is the sum of intensities of the center and side modes of a single emitter, $I_{\text {sat }}$ is the saturation intensity of a single emitter and $g_{0}$ the small signal gain, at and below the laser threshold.

In this appendix, we derive this expression starting from rate equations in the mean-field approximation as given, e.g., in Refs. [55, 56, 73].

Consider a laser medium with energy levels level 1 and level 2 (where level 1 has a lower energy than level 2), and let $N_{1}$ and $N_{2}$ be their respective (electron) population densities. As a result of pumping, radiative and nonradiative electronic transitions occur and one can derive a set of equations describing the change of population densities as Eq.(A.2) and Eq.(A.3), which are called rate equations. Pumping excites electrons from level 1 with a rate of $R_{1}$ and causes electrons to enter level 2 with a rate of $R_{2}$. Both levels have average lifetimes after which electrons spontaneously decay to a lower level (radiatively or non-radiatively), denoted as $\tau_{1}$ and $\tau_{2}$, for level 1 and 2 , respectively. Of course, electrons from level 2 can also decay to level 1, which is expressed by a lifetime $\tau_{21}$ (note that $\tau_{21}$ is included in $\tau_{2}$ ). So the rate of change of the population densities $N_{1}$ and $N_{2}$ due to pumping and decay are, respectively,

$$
\frac{d N_{1}}{d t}=-R_{1}-\frac{N_{1}}{\tau_{1}}+\frac{N_{2}}{\tau_{21}}
$$


and

$$
\frac{d N_{2}}{d t}=R_{2}-\frac{N_{2}}{\tau_{2}}
$$

For steady-state conditions $\left(d N_{1} / d t=0\right.$ and $\left.d N_{2} / d t=0\right)$ the common solution for Eq.(A.2) and Eq.(A.3) is represented as the difference between the two solutions: $N_{0}=N_{2}-N_{1}$. Where the subscript 0 denotes absence of both stimulated emission and absorption. The result is

$$
N_{0}=R_{2} \tau_{2}\left(1-\frac{\tau_{1}}{\tau_{21}}\right)+R_{1} \tau_{1}
$$

To include stimulated emission and absorption to the rate equations, the probability of the according transitions between level 1 and level 2 (and vice versa) take place is to be taken into account in Eqs.(A.2) and (A.3). This probability, $W_{i}$, can be expressed as

$$
W_{i}=\phi \cdot \sigma(\nu)
$$

where $\phi$ is the photon flux density, i.e., the number of photons per second and unit area that interacts with a medium. The second factor, $\sigma(\nu)$, is the cross section with which the light can interact. Including the induced transitions in the equations results in

$$
\begin{gathered}
\frac{d N_{1}}{d t}=-R_{1}-\frac{N_{1}}{\tau_{1}}+\frac{N_{2}}{\tau_{21}}+N_{2} W_{i}-N_{1} W_{i}, \\
\frac{d N_{2}}{d t}=R_{2}-\frac{N_{2}}{\tau_{2}}-N_{2} W_{i}+N_{1} W_{i} .
\end{gathered}
$$

For steady-state conditions $\left(d N_{1} / d t=0\right.$ and $\left.d N_{2} / d t=0\right)$ the common solution for Eq.(A.6) and Eq.(A.7) is represented as their difference $N=N_{2}-$ $N_{1}$, which gives

$$
N=\frac{N_{0}}{1+\tau_{s} W_{i}} .
$$

The implications of this steady-state solution will be shortly discussed after the individual terms in Eq.(A.8) are explained. In this expression,

$$
\tau_{s}=\tau_{2}+\tau_{1}\left(1-\frac{\tau_{2}}{\tau_{21}}\right)
$$

is called the saturation time constant, and it denotes the average time in which a photon is emitted per the cross sectional area by spontaneous emission, such that

$$
\sigma(\nu) \phi_{s}(\nu) \tau_{s}=1
$$


where $\phi_{s}(\nu)$ represents the photon flux density at which the gain coefficient decreases to half its maximum value. It is also called the saturation photon flux density. For the implications of Eq.(A.8), we can easily derive two distinct population differences. The first is in the absence of amplified radiation $\left(W_{i}=\right.$ 0 ), which means the population difference is $N=N_{0}$. The second is for a very large probability $W_{i}$, when stimulated emission and stimulated absorption dominate the interaction between the energy levels, which means the population difference is $N=0$. This is called saturation of the gain.

Inserting Eq.(A.5) into Eq.(A.8) leads to

$$
N=\frac{N_{0}}{1+\tau_{s} \phi \sigma(\nu)} .
$$

Inserting Eq.(A.10) into Eq.(A.11) gives

$$
N=\frac{N_{0}}{1+\phi / \phi_{s}} .
$$

To express Eq.(A.12) in terms of gain, we insert the definition of the gain coefficient, $g=N \cdot \sigma(\nu)$ and $g_{0}=N_{0} \cdot \sigma(\nu)$. And to express Eq.(A.12) in terms of intensity, we insert the definition of the photon flux, $\phi_{t o t}=I_{t o t} /(h \nu)$ and $\phi_{\text {sat }}=I_{\text {sat }} /(h \nu)$, which yields

$$
g\left(I_{t o t}\right)=\frac{g_{0}}{1+I_{\text {tot }} / I_{\text {sat }}} .
$$





\section{Bibliography}

[1] D. Marpaung, L. Zhuang, M. Burla, C. Roeloffzen, J. Verpoorte, H. Schippers, A. Hulzinga, P. Jorna, W.P. Beeker, A. Leinse, R.G. Heideman, B. Noharet, Q. Wang, B. Sanadgol, and R. Baggen, "Towards a broadband and squint-free $\mathrm{Ku}$-band phased array antenna system for airborne satellite communications," Proc. of the 5th European Conference on Antennas and Propagation (EUCAP) pp. 2623-2627 (2011).

[2] A. Meijerink, C.G.H. Roeloffzen, R. Meijerink, L. Zhuang, D.A.I. Marpaung, M.J. Bentum, M. Burla, J. Verpoorte, P. Jorna, A. Hulzinga, and W. van Etten, "Novel ring resonator-based integrated photonic beamformer for broadband phased array receive antennasPart I: Design and performance analysis," J. Lightw. Technol. 28, 3-18 (2010).

[3] L. Zhuang, C.G.H. Roeloffzen, A. Meijerink, M. Burla, D.A.I. Marpaung, A. Leinse, M. Hoekman, R.G. Heideman, and W. van Etten, "Novel ring resonator-based integrated photonic beamformer for broadband phased array receive antennasPart II: Experimental prototype," Journal of Lightwave Technology 28, 19-31 (2010).

[4] T.H. Maiman, "Stimulated Optical Radiation in Ruby," Nature 187, 493494 (1960).

[5] Website Samsung, a manufacturer of, a.o., solid state drives, http://www. samsung. com/nl/ssd/ (2012).

[6] IEEE, IEEE SA - 802.3-2012 - IEEE Approved Draft Standard for Ethernet (2012).

[7] Website KPN, a Dutch telecommunication provider, http://www.kpn.com/prive/internet/glasvezel-internet.htm (2012).

[8] F. Hansteen, A. Kimel, A. Kirilyuk, and T. Rasing, "Femtosecond Photomagnetic Switching of Spins in Ferrimagnetic Garnet Films," Physical Review Letters 95, 047402 (2005).

[9] P.J. Harding, T.G. Euser, and W.L. Vos, "Identification of competing ultrafast all-optical switching mechanisms in Si woodpile photonic crystals," Journal of the Optical Society of America. B 26, 610-619 (2009). 
[10] E. Yüce, G. Ctistis, J. Claudon, E. Dupuy, R. D. Buijs, B. de Ronde, A. P. Mosk, J.-M. Gérard, and W.L. Vos, "All-optical Switching of a Microcavity Repeated at Terahertz Rates," (Submitted). http://arxiv.org/abs/1211.1865

[11] P. Signoret, M. Myara, J.P. Tourrenc, B. Orsal, M.H. Monier, J. Jacquet, P. Leboudec, and F. Marin, "Bragg section effects on linewidth and lineshape in 1.55- $\mu \mathrm{m}$ DBR tunable laser diodes," IEEE Photonics Technology Letters 16, 1429-1431 (2004).

[12] C.K. Laue, R. Knappe, K.-J. Boller, and R. Wallenstein, "Wavelength tuning and spectral properties of distributed feedback diode lasers with a short external optical cavity," Applied Optics 40, 3051-3059 (2001).

[13] HL Stover and WH Steier, "Locking of laser oscillators by light injection," Applied Physics Letters 8, 91-93 (1966).

[14] U. Keller and A.C. Tropper, "Passively Modelocked Surface-Emitting Semiconductor Lasers," Physics Reports 429, 67-210 (2006).

[15] R.M. Oldenbeuving, C.J. Lee, P.D. van Voorst, H.L. Offerhaus, and K.-J. Boller, "Modeling of mode locking in a laser with spatially separate gain media," Optics Express 18, 22 996-23008 (2010).

[16] E. Ip, A.P.T. Lau, D.J. Barros, and J.M. Kahn, "Coherent detection in optical fiber systems: erratum," Opt. Express 16, 21 943-21943 (2008).

[17] E. Bruce, "Tunable lasers," IEEE Spectrum 39, 35-39 (2002).

[18] S.D. Saliba and R.E. Scholten, "Linewidths below $100 \mathrm{kHz}$ with external cavity diode lasers," Applied optics 48, 6961-6966 (2009).

[19] C. Henry, "Theory of the linewidth of semiconductor lasers," IEEE Journal of Quantum Electronics 18, 259-264 (1982).

[20] T. Chu, N. Fujioka, and M. Ishizaka, "Compact, low-power-consumption wavelength tunable laser fabricated with silicon photonic-wire waveguide micro-ring resonators," Optics Express 17, 14063-14068 (2009).

[21] N. Fujioka, T. Chu, and M. Ishizaka, "Compact and low power consumption hybrid integrated wavelength tunable laser module using silicon waveguide resonators," Journal of Lightwave Technology 28, 3115-3120 (2010).

[22] F. Morichetti, A. Melloni, M. Martinelli, R.G. Heideman, A. Leinse, D.H. Geuzebroek, and A. Borreman, "Box-shaped dielectric waveguides: A new concept in integrated optics?" Journal of Lightwave Technology 25, 25792589 (2007). 
[23] M. Saruwatari and K. Nawata, "Semiconductor laser to single-mode fiber coupler," Applied Optics 18, 1847-1856 (1979).

[24] P. Zorabedian, "Axial-mode instability in tunable external-cavity semiconductor lasers," IEEE Journal of Quantum Electronics 30, 1542-1552 (1994).

[25] A. Yariv, "Universal relations for coupling of optical power between microresonators and dielectric waveguides," Electronics Letters 36, 321-322 (2000).

[26] D.H. Geuzebroek, E.J. Klein, H. Kelderman, F.S. Tan, D.J.W. Klunder, and A. Driessen, "Thermally tuneable, wide FSR switch based on micro-ring resonators," Proc. Symposium IEEE/LEOS Benelux pp. 155158 (2002).

[27] R. Grover, V. Van, T.A. Ibrahim, P.P. Absil, L.C. Calhoun, F.G. Johnson, J.V. Hryniewicz, and P.T. Ho, "Parallel-cascaded semiconductor microring resonators for high-order and wide-FSR filters," Journal of Lightwave Technology 20, 900-905 (2002).

[28] B.E. Little, S.T. Chu, H.A. Haus, J. Foresi, and J.P. Laine, "Microring resonator channel dropping filters," Journal of Lightwave Technology 15, 998-1005 (1997).

[29] G.M. Stéphan, T.T. Tam, S. Blin, P. Besnard, and M. Têtu, "Laser line shape and spectral density of frequency noise," Physical Review A 71, 043809 (2005).

[30] A.L. Schawlow and C.H. Townes, "Infrared and optical masers," Physical Review 112, 1940-1949 (1958).

[31] A. Driessen, R. Dekker, M.B.J. Diemeer, D.H. Geuzebroek, H.J.W.M. Hoekstra, E.J. Klein, and A. Leinse, "Microresonators as promising building blocks for VLSI photonics," Proceedings of SPIEIntegrated Optics: Theory and Applications pp. 59560Q1-59560Q14 (2005).

[32] P. Laurent, A. Clairon, and C. Bréant, "Frequency noise analysis of optically self-locked diode lasers," IEEE Journal of Quantum Electronics 25, 1131-1142 (1989).

[33] R.G. Heideman, A. Leinse, W. Hoving, R. Dekker, D.H. Geuzebroek, E.J. Klein, R. Stoffer, C. Roeloffzen, L. Zhuang, and A. Meijerink, "Largescale integrated optics using TriPleX ${ }^{T M}$ waveguide technology: from UV to IR," Proc. of SPIE 7221, 72 210R-1 (2009).

[34] A. Yariv, "Critical coupling and its control in optical waveguide-ring resonator systems," IEEE Photonics Technology Letters 14, 483-485 (2002). 
[35] E.J. Klein, Densely Integrated Microring-Resonator Based Components for Fiber-To-The-Home Applications, Ph.D. thesis, University of Twente, Enschede (2007).

http://dx.doi.org/10.3990/1.9789036524957

[36] R. Adler, "A study of locking phenomena in oscillators," Proceedings of the IRE 34, 351-357 (1946).

[37] F. Mogensen, H. Olesen, and G. Jacobsen, "Locking conditions and stability properties for a semiconductor laser with external light injection," IEEE Journal of Quantum Electronics 21, 784-793 (1985).

[38] U. Gliese, E.L. Christensen, and K.E. Stubkjaer, "Laser linewidth requirements and improvements for coherent optical beam forming networks in satellites," Journal of Lightwave Technology 9, 779-790 (1991).

[39] H.L. Stover, "Theoretical explanation for the output spectra of unlocked driven oscillators," Proceedings of the IEEE 54, 310-311 (1966).

[40] Q. Xu, J. Shakya, and M. Lipson, "Direct measurement of tunable optical delays on chip analogue to electromagnetically induced transparency," Opt. Express 14, 6463-6468 (2006).

[41] R.W. Boyd and D.J. Gauthier, "Photonics: transparency on an optical chip," Nature 441, 701-702 (2006).

[42] (2010,2011), waveguide mask design was done at XiO Photonics, mask fabrication at MiPlaza, Philips.

[43] LioniX BV, http://www.lionixbv.com.

[44] XiO Photonics BV, http://www.xiophotonics.com.

[45] MESA+ institute for nanotechnology, http://www . utwente.nl/mesaplus/.

[46] H. Tsuchida, "Simple technique for improving the resolution of the delayed self-heterodyne method," Optics letters 15, 640-642 (1990).

[47] M. Han and A. Wang, "Analysis of a loss-compensated recirculating delayed self-heterodyne interferometer for laser linewidth measurement," Applied Physics B: Lasers and Optics 81, 53-58 (2005).

[48] J.K.S. Poon, J. Scheuer, Y. Xu, and A. Yariv, "Designing coupledresonator optical waveguide delay lines," JOSA B 21, 1665-1673 (2004).

[49] R. Kazarinov and C. Henry, "The relation of line narrowing and chirp reduction resulting from the coupling of a semiconductor laser to passive resonator," IEEE Journal of Quantum Electronics 23, 1401-1409 (1987).

[50] J. Klein and J.D. Kafka, "The Ti:Sapphire laser: The flexible research tool," Nature Photonics 4, 289 (2010). 
[51] U. Keller, "Recent Developments in Compact Ultrafast Lasers," Nature 424, 831-838 (2003).

[52] T. Pfeiffer and G. Veith, "40 GHz Pulse Generation Using A Widely Tunable All-Polarization Preserving Erbium Fiber Ring Laser," Electronics Letters 29, 1849-1850 (1993).

[53] A. Robertson, M.E. Klein, M.A. Tremont, K.-J. Boller, and R. Wallenstein, "2.5-GHz repetition-rate singly resonant optical parametric oscillator synchronously pumped by a mode-locked diode oscillator amplifier system," Optics Letters 25, 657-659 (2000).

[54] A. Aschwanden, D. Lorenser, H.J. Unold, and R. Paschotta, "10 GHz Passively Mode-Locked External-Cavity Semiconductor Laser With 1.4 W Average Output Power," Applied Physics Letters 86, 131102 (2005).

[55] A.E. Siegman, Lasers (University Science Books, 1986), chapters 7 and 8.

[56] O. Svelto, Principles of Lasers (Plenum Press New York, 1998), chapter 8.

[57] U. Keller, K.J. Weingarten, F.X. Kärtner, D. Kopf, B. Braun, I.D. Jung, R. Fluck, C. Hönninger, N. Matuschek, and J. Aus der Au, "Semiconductor Saturable Absorber Mirrors (SESAM's) for Femtosecond to nanosecond Pulse Generation in Solid-State Lasers," IEEE Journal Of Selected Topics In Quantum Electronics 2, 435-453 (1996).

[58] R. Grange, M. Haiml, R. Paschotta, G.J. Spühler, L. Krainer, O. Ostinelli, M. Golling, and U. Keller, "New Regime Of Inverse Saturable Absorption For Selfstabilizing Passively Modelocked Lasers," Applied Physics B 80, 151-158 (2005).

[59] A.H. Quarterman, A. Perevedentsev, K.G. Wilcox, V. Apostolopoulos, H.E. Beere, I. Farrer, D.A. Ritchie, and A.C. Tropper, "Passively harmonically mode-locked vertical-external-cavity surface-emitting laser emitting 1.1 ps pulses at $147 \mathrm{GHz}$ repetition rate," Applied Physics Letters 97, 251 101-251101 (2010).

[60] K.G. Wilcox, A.H. Quarterman, V. Apostolopoulos, H.E. Beere, I. Farrer, D.A. Ritchie, and A.C. Tropper, "175 GHz, 400-fs-pulse harmonically mode-locked surface emitting semiconductor laser." Optics Express 20, 7040-7045 (2012).

[61] R.A. Rooth, F.A. van Goor, and W.J. Witteman, "An Independently Adjustable Multiline AM Mode-Locked TEA CO2 Laser," IEEE Journal of Quantum Electronics QE-19, 1610-1612 (1983).

[62] V. Daneu, A. Sanchez, T.Y. Fan, H.K. Choi, G.W. Turner, and C.C. Cook, "Spectral Beam Combining Of A Broad-Stripe Diode Laser Array In An External Cavity," Optics Letters 25, 405-407 (2000). 
[63] M.J.R. Heck, E.A.J.M. Bente, Y. Barbarin, D. Lenstra, and M.K. Smit, "Simulation and Design of Integrated Femtosecond Passively Mode-Locked Semiconductor Ring Lasers Including Integrated Passive Pulse Shaping Components," IEEE Journal Of Selected Topics In Quantum Electronics 12, 265-276 (2006).

[64] T.W. Hänsch, "A proposed sub-femtosecond pulse synthesizer using separate phase-locked laser oscillators," Optics Communications 80, 71-75 (1990).

[65] D.C. Byrne, W.H. Guo, Q.Y. Lu, and J.F. Donegan, "Broadband reflection method to measure waveguide loss," Electronic Letters 45, 322-323 (2009).

[66] N. Trela, H.J. Baker, R. McBride, and D.R. Hall, "Low-Loss Wavelength Locking of a 49-Element Single Mode Diode Laser Bar with Phase-Plate Beam Correction," Conference proceedings CLEO Europe - EQEC 2009 (2009).

[67] L.E. Eng, D.G. Mehuys, M. Mittelstein, and A. Yariv, "Broadband Tuning (170 nm) of InGaAs Quantum Well Lasers," Electronics Letters 26, 16751677 (1990).

[68] BATOP-GmbH, "BATOP website, a SESAM manufacturer," http://www. batop.com (2010).

[69] K.M. Garner and G.A. Massey, "Laser Mode Locking By Active External Modulation," IEEE Journal of Quantum Electronics 28, 297-301 (1992).

[70] J.C.M. Diels, J.J. Fontaine, I.C. McMichael, and F. Simoni, "Control and measurement of ultrashort pulse shapes (in amplitude and phase) with femtosecond accuracy," Applied Optics 24, 1270-1282 (1985).

[71] R. Trebino, Frequency-resolved optical gating: the measurement of ultrashort laser pulses (Springer, 2000).

[72] J.F. Bauters, M.J.R. Heck, D.D. John, J.S. Barton, C.M. Bruinink, A. Leinse, R.G. Heideman, D.J. Blumenthal, and J.E. Bowers, "Planar waveguides with less than $0.1 \mathrm{~dB} / \mathrm{m}$ propagation loss fabricated with wafer bonding," Optics Express 19, 24 090-24101 (2011).

[73] B.E.A. Saleh and M.C. Teich, Fundamentals of Photnics, Wiley Series in Pure and Applied Optics (John Wiley \& Sons, Inc., 2007), second edition edn., chapters 13 and 14 . 


\section{Publications}

- Papers

- R.M. Oldenbeuving, C.J. Lee, P.D. van Voorst, H.L. Offerhaus, K.J. Boller, Modeling of mode locking in a laser with spatially separate gain media, Opt. Exp. 18, 22996 (2010)

- R.M. Oldenbeuving, E.J. Klein, H.L. Offerhaus, C.J. Lee, H. Song, K.-J. Boller, $25 \mathrm{kHz}$ narrow spectral bandwidth of a wavelength tunable diode laser with a short waveguide-based external cavity, Laser Phys. Lett. 10, 015804 (2013)

- R.M. Oldenbeuving, M.R.H. Kahn, C.G.H. Roeloffzen, E.J. Klein, C.J. Lee, H.L. Offerhaus, K.-J. Boller, Injection locking of a $W E C S L$, in preparation

- R.M. Oldenbeuving, H. Song, G. Schitter, M. Verhaegen, E.J. Klein, C.J. Lee, H.L. Offerhaus, K.-J. Boller, Simple wavemeter in integrated optics, achieving high precision by using neural network, in preparation

- Oral presentations

- R.M. Oldenbeuving, Mode-locking of wavelength combined lasers, both in free space and integrated on a chip, MESA+ colloquium, 13-01-2009, Enschede, The Netherlands

- R.M. Oldenbeuving, Waveguide based external cavity semiconductor lasers, Philips research labs (dept. Light generation), 04-09-2009, Aachen, Germany

- R.M. Oldenbeuving, Waveguide based external cavity semiconductor lasers, Fraunhofer Institute für Lasertechnologie ILT, 04-09-2009, Aachen, Germany

- R.M. Oldenbeuving, Hitting the right notes- optical pulse synthesis, Applied Nano Photonics annual retreat, 28-05-2010, Bad Bentheim, Germany

- R.M. Oldenbeuving, E.J. Klein, C.J. Lee, H.L. Offerhaus, H. Song, M. Verhaegen, K.-J. Boller, Waveguide-based External Cavity Semiconductor Lasers, First Smart Optical Systems annual symposium, 14-06-2010, Scheveningen, The Netherlands 
- R.M. Oldenbeuving, P.D. van Voorst, C.J. Lee, H.L. Offerhaus, K.J. Boller, Separate gain media, an alternative approach to modelocking, 34 ${ }^{\text {th }}$ Annual Meeting NNV AMO Lunteren, 13-10-2010, Lunteren, The Netherlands

- R.M. Oldenbeuving, E.J. Klein, C.J. Lee, H.L. Offerhaus, H. Song, M. Verhaegen, K.-J. Boller, Waveguide-based External Cavity Semiconductor Lasers, Second Smart Optical Systems annual symposium, 12-09-2011, Delft, The Netherlands

- R.M. Oldenbeuving, H. Song, M. Verhaegen, E.J. Klein, C.J. Lee, G. Schitter, H.L. Offerhaus, K.-J. Boller, Waveguide-based External Cavity Diode Laser with method of controlling spectral properties, $1^{\text {st }}$ EOS Topical Meeting on Micro- and Nano- Optoelectronic Systems, 09-12-2011, Bremen, Germany

- Poster presentations

- R.M. Oldenbeuving, E.J. Klein, H.L. Offerhaus, C.J. Lee, K.J. Boller, M. Verhaegen, Waveguide-based External Cavity Semiconductor Laser Arrays, A Smart Optical Systems project, IOP photonics begeleidersdag, 06-11-2009, Utrecht, The Netherlands

- R.M. Oldenbeuving, E.J. Klein, H.L. Offerhaus, C.J. Lee, K.J. Boller, M. Verhaegen, Waveguide-based External Cavity Semiconductor Laser Arrays, A Smart Optical Systems project, Kickoff symposium Applied Nano Photonics, 03-12-2009, Enschede, The Netherlands

- R.M. Oldenbeuving, E.J. Klein, H.L. Offerhaus, C.J. Lee, K.J. Boller, M. Verhaegen, Waveguide-based External Cavity Semiconductor Laser Arrays, Physics@FOM , 20-01-2010, Veldhoven, The Netherlands

- R.M. Oldenbeuving, E.J. Klein, H.L. Offerhaus, C.J. Lee, K.J. Boller, M. Verhaegen, Waveguide-based External Cavity Semiconductor Laser Arrays, SUSSP66 Summerschool on Ultrafast Nonlinear Optics, 16-08-2010, Edinburgh, United Kingdom

- R.M. Oldenbeuving, C.J. Lee, H.L. Offerhaus, K.-J. Boller, Modeling a SEGA mode-locked laser, Physics@FOM, 19-01-2011, Veldhoven, The Netherlands

- R.M. Oldenbeuving, E.J. Klein, H.L. Offerhaus, C.J. Lee, K.J. Boller, M. Verhaegen, Waveguide-based External Cavity Semiconductor Laser Arrays, A Smart Optical Systems project, IOP photonics begeleidersdag, 06-11-2009, Utrecht, The Netherlands

- R.M. Oldenbeuving, E.J. Klein, H.L. Offerhaus, C.J. Lee, H. Song, M. Verhaegen, K.-J. Boller, Tunable WECSL with $25 \mathrm{kHz}$ narrow linewidth, STW-IOP meeting, 09-11-2011, Utrecht, The Netherlands 
- R.M. Oldenbeuving, H. Song, M. Verhaegen, E.J. Klein, C.J. Lee, G. Schitter, H.L. Offerhaus, K.-J. Boller, Waveguide-based External Cavity Diode Laser with method of controlling spectral properties, Physics@FOM, 18-01-2012, Veldhoven, The Netherlands

- R.M. Oldenbeuving, H.L. Offerhaus, C.J. Lee, K.-J. Boller, SEGA mode locking: first experimental results, $36^{\text {th }}$ Annual Meeting NNV AMO Lunteren, 09-10-2012, Lunteren, The Netherlands

- R.M. Oldenbeuving, E.J. Klein, H.L. Offerhaus, C.J. Lee, M. Verhaegen, K.-J. Boller, Waveguide-based External Cavity Semiconductor Lasers, Third Smart Optical Systems annual symposium, 31-102012, Leiden, The Netherlands

- R.M. Oldenbeuving, M.R.H. Khan, E.J. Klein, C.J. Lee, H.L. Offerhaus, C.G.H. Roeloffzen, K.-J. Boller, Waveguide-based External Cavity Semiconductor Laser, Physics@FOM, 22-01-2013, Veldhoven, The Netherlands

- Conference proceedings

- R.M. Oldenbeuving, E.J. Klein, C.J. Lee, H.L. Offerhaus, H. Song, M. Verhaegen, K.-J. Boller, Waveguide-based External Cavity Semiconductor Lasers, First Smart Optical Systems annual symposium, 14-06-2010, Scheveningen, The Netherlands

- R.M. Oldenbeuving, E.J. Klein, C.J. Lee, H.L. Offerhaus, H. Song, M. Verhaegen, K.-J. Boller, Waveguide-based External Cavity Semiconductor Lasers, Second Smart Optical Systems annual symposium, 12-09-2011, Delft, The Netherlands

- R.M. Oldenbeuving, E.J. Klein, H.L. Offerhaus, C.J. Lee, M. Verhaegen, K.-J. Boller, Waveguide-based External Cavity Semiconductor Lasers, Third Smart Optical Systems annual symposium, 31-102012, Leiden, The Netherlands

- Other publications

- R.M. Oldenbeuving, C.J. Lee, H.L. Offerhaus, K.-J. Boller, SEGA mode locking, Arago Focus, jaargang 41, nummer 4, pp. 19 (2011) 



\section{Dankwoord}

Aan alles komt een eind. U bent nu aanbeland aan het einde van mijn proefschrift. Dit proefschrift vormt het einde van mijn vierjarige promotietraject. Dit promotietraject vormt op zijn beurt weer het einde van een bijna twaalfjarige carrière aan de Universiteit Twente.

Boven alles wil ik m'n ouders bedanken. Zonder hun onvoorwaardelijke steun had ik nooit zo ver kunnen komen. Maar ook mijn broer, schoonzus en twee jonge nichtjes wil ik niet onbenoemd laten.

Twaalf jaar op dezelfde universiteit en daarbij succesvol een studie en promotie afronden red je natuurlijk niet zonder hulp. Daarom wil ik graag beginnen met het bedanken van mijn professor en promotor Klaus Boller voor alle waardevolle wetenschappelijke discussies die we hebben gevoerd. In het bijzonder wil ik hem graag bedanken voor het nakijken en corrigeren van mijn proefschrift, wat ons beiden tot in de late uurtjes bezig heeft gehouden. Ook Herman Offerhaus en Chris Lee hebben zich flink ingezet om mijn promotie tot een succesvol einde te brengen, dus hen wil ik graag bedanken voor dagelijkse begeleiding en advies.

Voordat ik alle andere mensen ga bedanken waarmee ik heb samengewerkt, wil ik nog even een speciaal dankwoord uitspreken richting Studievereniging Arago. Vlak na mijn afstuderen wisten ze mij te verrassen met een Lidmaatschap van Verdienste voor mijn inzet voor de vereniging. Maar laten we eerlijk zijn; ik had me natuurlijk nooit ingezet als Arago een saaie en slechte omgeving voor studenten zou zijn. Dus Ome Arago: bedankt voor het creëren van een atmosfeer waar studenten zich kunnen ontplooien naast hun studie, op zo'n manier dat hun studie er beter van wordt. Keep up the good work.

$\mathrm{Nu}$ het lijstje van collega's en mensen met wie ik heb samengewerkt om mijn proefschrift mogelijk te maken. Allereerst mijn directe collega en goede vriend Thomas, die na het aflopen van zijn contract zo snel mogelijk naar het buitenland wilde vertrekken (lees: 1 februari, de dag van mijn promotie, zit hij in het vliegtuig). Mijn paranimfen Jörn en Robert, met wie ik met veel plezier heb samengewerkt. Extra bedankje voor Robert, voor het maken van de SEM afbeelding die ik heb gebruikt als voorkant van mijn proefschrift. Verder mijn huidige en voormalige collega's Kasper, Muharrem, Joan, Jean, Yin, Jonathan, Bert, PeterS, FredG, FredB, Ab, Gerard, Piet, Simone, Carin, PeterV, Mark, Rolf, Arie, Willem, Kai, Rob, Wiebke, Marc, zonder wie het werk een stuk minder leuk geweest zou zijn. 
Daarnaast kon ik mijn PhD niet doen zonder de hulp van de bedrijven LioniX en XiO Photonics. Ik heb samen met hen mijn projectvoorstel geschreven, waarbij vooral $\mathrm{XiO}$ Photonics veel hulp en industri'ele bijdrage heeft geleverd om het voorstel mogelijk te maken. Ook tijdens mijn promotie heb ik veel met $\mathrm{XiO}$ samengewerkt, en heb ik veel gehad aan de nuttige discussies en helpende handen. Ik wil in het bijzonder de volgende mensen wil bedanken, met wie ik veel heb samengewerkt. Willem, Edwin, Douwe, Ronald, Dimitri, Hans, René en Arne.

Mijn project bestond niet alleen uit werk in Twente, maar het was een samenwerkingsverband met de TU Delft. Hier wil ik Hong, Tope en Michel bedanken voor de prettige samenwerking.

I would also like to use this opportunity to thank my colleagues at the Cambridge University (UK), for their help in the SEGA mode locking experiments. With their expertise we were able to use different types of saturable absorbers other than SESAMs, based on carbon nanotubes and graphene flakes.

Naast alle collega's heb ik ook nog een waslijst aan vrienden te bedanken, zonder wie het leven bijzonder saai zou zijn. Allereerst zijn daar mijn oud flatgenoten van Campuslaan 67. Zelfs na vele jaren geleden afscheid te hebben genomen en te zijn verspreid over heel Nederland, voelt het nog steeds of we nooit weg zijn geweest wanneer we elkaar weer tegenkomen. Bijzonder bedankt aan de mensen met wie ik heb samengewoond en veel plezier heb mogen beleven: Steven, Tom, Sikh, Chris, Karin, Ale, Roy, Edwin, Bart, Joost, Nico, Stijn, Monique, Sophie, Wouter, Pieter, Joost en alle anderen.

Ook heb ik ruim twee jaar geleden een nieuwe sport opgepakt: Ultimate Frisbee. Een erg leuke, dynamische, energieke en fysiek uitdagende sport, met bijzonder leuke en gastvrije mensen. Ik wil de Disc Devils Twente daarom bedanken voor ruim twee jaar actieve sport op hoog niveau en voor gezellige niet-sport gerelateerde activiteiten. Ik hoop dit in de toekomst nog te kunnen doorzetten.

Zoals gezegd, aan alles komt een eind. Dit proefschrift, 12 jaar UT, m'n promotie, etc. Maar dat betekent ook dat er steeds een nieuw begin is. Op naar de volgende uitdaging!

Cheers,

Ruud. 